Pacific Journal of Mathematics

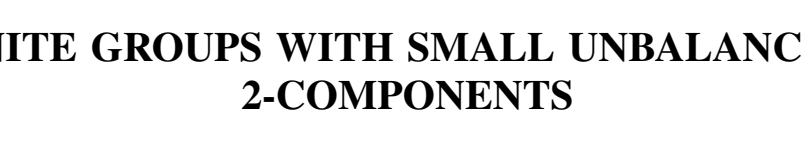




\title{
FINITE GROUPS WITH SMALL UNBALANCING 2-COMPONENTS
}

\author{
Robert GILMAN AND RONALd SOLOMON
}

In this paper we supply one link in a chain of results which will prove the following two conjectures:

$B(G)$-Conjecture. If $H$ is a 2-local subgroup of a finite group $G$, then $[L(H), O(H)] \subseteq O(G)$.

Unbalanced Group Conjecture. If $G$ is a finite group with $O\left(C_{G}(t)\right) \varsubsetneqq O(G)$ for some involution $t \in G$, then $O\left(C_{G}(t)\right)$ acts nontrivially on $L / Z^{*}(L)$ where $L$ is a 2-component of $G$ with $L / Z^{*}(L)$ isomorphic to one of the following simple groups:

(1) A simple Chevalley group or twisted variation over a field of odd order;

(2) An alternating group of odd degree;

(3) PSL $(3,4)$ of $\mathrm{He}$, the simple group of Held.

In 1973 John Thompson, inspired by the Standard Component Theorem of Michael Aschbacher, formulated the $B(G)$-Conjecture. Daniel Gorenstein and John Walter had previously verified that the $B(G)$-Conjecture held in a wide variety of circumstances, but they had not formulated a general conjecture. The history of the $B(G)$ Conjecture is discussed in Chapter VI of Gorenstein's survey article, The classification of finite simple groups, I, II, which will appear as a monograph of the American Mathematical Society.

In 1974 converstions among Michael Aschbacher, David Goldschmidt, John Thompson, and John Walter directed attention toward the more general Unbalanced Group Conjecture. In $\S 4$ we show how these various results fit together and how completion of some standard component problems will supply the missing link. Before discussing our work further we set up some notation.

For any finite group $G, O(G)$ is the largest normal subgroup of odd order, $\mathrm{O}_{2}(G)$ is the largest normal 2-subgroup, $O_{2^{\prime}, 2}(G)$ is the inverse image in $G$ of $O_{2}(G / O(G))$, and $Z^{*}(G)$ is the inverse image in $G$ of $Z(G / O(G))$. $\operatorname{Syl} p(G)$ is the set of Sylow $p$-subgroups of $G$, and $\operatorname{Inv}(G)$ is the set of involutions of $G$. The 2-rank of $G$ is denoted by $m(G)$.

$G$ is quasisimple if $G$ is perfect and $G / Z(G)$ is simple. If $G$ is simple, $\hat{G}$ stands for any quasisimple extension of $G$, (including $G$ itself). A component of $G$ is a subnormal quasisimple subgroup, and a 2-component, $J$, of $G$ is a perfect subnormal subgroup with $J / O(J)$ quasisimple. $L(G)$ is the product of all 2-components of $G, F(G)$ is the fitting subgroup of $G, E(G)$ is the product of all components of 
$G$, and $F^{*}(G)=E(G) F(G)$. The properties of components and 2-components are discussed in [2], [8], [13] and [26]. In particular each 2-component of $G$ is normal in $L(G)$ by [13, $\S 2]$.

A 2-component $J$ of $C_{G}(a), a \in \operatorname{Inv}(g)$, is standard in $G$ if

(1) $J$ is quasisimple;

(2) $\left[J, J^{g}\right] \neq 1$ for all $g \in G$;

(3) If $g \in G$ with $\left|C_{G}\left(\left\langle J, J^{g}\right\rangle\right)\right|$ even, then $J=J^{g}$. The standard component (or standard form) problem for $J$ is to identify $\left\langle K^{H}\right\rangle$ whenever $K$ is a standard component of $H$ and $K$ is isomorphic to $J$.

If a 2-group $A$ acts on a group $N$, define $N_{A}=L\left(C_{N}(A)\right)$. For cyclic groups write $N_{\langle a\rangle}$ as $N_{a}$. By [13, $\S 3$ and 4] we know that $N_{A} \subseteq L(N)$. Further if $A$ and $B$ are 2-subgroups of $G$ which normalize each other, and if $J$ is a 2-component of $N_{G}(A)$ with $[J, B] \subseteq O(J)$, then $J_{B}=K_{A}$ where $K=\left\langle L^{A}\right\rangle$ for some 2-component $L$ of $N_{G}(B)$. We refer to these results as $L$-Balance and we say that $L$ corresponds to $J$. If $L=\left\langle L^{A}\right\rangle$ and $J_{B}$ covers $L / O(L)$, then $J$ also corresponds to $L$, and we will say in this case that $J$ and $L$ correspond isomorphically.

If $a$ and $b$ are commuting involutions of $G$ and if $J$ and $K$ are 2-components of $C_{G}(a)$ and $C_{G}(b)$ respectively such that $K$ corresponds to $J$ (and necessarily $[J, b] \subseteq O(J)$ ), we will write $J \rightarrow K$. We define a relation $\rightarrow$ on the set of 2-components of centralizers of involutions of $G$ by $L \rightarrow M$ if and only if $L=L_{1} \rightarrow L_{2} \rightarrow \cdots \rightarrow L_{n}=M$ for some sequence of 2-components. $J$ is maximal with respect to $G$ if and only if $J \rightarrow M$ implies $J / Z^{*}(J) \cong M / Z^{*}(M)$.

Finally an unbalanced group is one satisfying the hypothesis of what we shall refer to for brevity as the U.G.-Conjecture.

Now we return to our discussion of the context of this paper. A short argument using $L$-Balance together with the properties of the simple groups listed in the U.G.-Conjecture shows that the U.G.Conjecture implies the $B(G)$-Conjecture. The advantage of the former is the inductive leverage provided by the following lemma, which incorporates results of Gorenstein and Walter [13], Gorenstein and Harada [12], and Aschbacher [1].

LEMMA 1.1. Let $G$ be an unbalanced group. Either $G$ satisfies the Unbalanced Group Conjecture or $G$ possesses a pair of commuting involutions $(a, x)$, such that for some 2-component $J$ of $C_{G}(a)$

$(*)$

$$
\left[J, O\left(C_{G}(x)\right) \cap C_{G}(a)\right]=J=[J, x] .
$$

By an unbalancing triple of $G$ we mean a triple $(a, x, J)$ with $a, x, J$ as in Lemma 1.1 and satisfying $\left({ }^{*}\right)$. We refer to $J$ as an 
unbalancing 2-component of $G . G$ is a minimal unbalanced group if every proper section of $G$ satisfies the $U . G$-Conjecture and $G$ has an unbalancing triple. By Lemma 1.1 it suffices to prove the U.G.Conjecture for minimal unbalanced groups.

We catalog the current work on the U.G.-Conjecture.

THeOREM 1.2 (Aschbacher [4]). Let G be a finite group with $F^{*}(G)$ quasisimple. Suppose that $a \in \operatorname{Inv}(G), J$ is a 2-component of $C_{G}(a)$, $m(J)=1$, and $a \in J$. Then $F^{*}(G)$ is isomorphic to a known group; in particular if $G$ is unbalanced, $F^{*}(G) / Z(G)$ is isomorphic to a Chevalley group or twisted variation over a field of odd order.

TheOREM 1.3 (Aschbacher [3], Solomon [25], [27], [28]). Let G be a finite group with $F^{*}(G)$ quasisimple. Suppose that $a \in \operatorname{Inv}(G)$ and $J$ is a 2-component of $C_{G}(a)$ with $J / Z^{*}(J) \cong A_{n}$ for some odd $n \geqq 9$. Then $F^{*}(G) / Z(G)$ is isomorphic to $A_{m}$ for some odd $m$ or to Lyons' simple group.

Theorem 1.4 (Thompson, Burgoyne [5], Griess, Solomon [14, Theorem 2.22]). Let $G$ be a minimal unbalanced group with $F^{*}(G)$ quasisimple. Suppose that $a \in \operatorname{Inv}(G)$ and $J$ is an unbalancing 2component of $C_{G}(a)$ with $J / Z^{*}(J)$ isomorphic to a simple Chevalley group or twisted variation over a finite field of odd order. Suppose further that $J / O(J) \supsetneqq L_{2}(q)$. Then $G$ satisfies the conclusion of Theorem 1.2 or Theorem 1.3 .

Theorem 1.5 (Harris, Solomon $[16,18]$ ). Let $G$ be a finite group with $F^{*}(G)$ quasisimple. Suppose that $a \in \operatorname{Inv}(G)$ and $J$ is a 2-component of $C_{G}(a)$ with a Sylow 2-subgroup isomorphic to $D_{8}$. Suppose that $C_{G}(J / O(J))$ has cyclic Sylow 2-subgroups. Then $F^{*}(G)$ is isomorphic to one of the following simple groups:

(1) $A_{8}, \operatorname{Sp}(4,4), L_{5}(2), U_{5}(2)$ or $H i S$ with $J / O(J) \cong A_{6}$;

(2) $A_{9}$ or $\mathrm{He}$ with $\mathrm{J} / \mathrm{O}(\mathrm{J}) \cong A_{7}$;

(3) $L_{3}(4)$ or $H J$ with $J / O(J) \cong L_{2}(7)$;

(4) $L_{2}\left(q^{2}\right), L_{3}(q)$ or $U_{3}(q)$ with $J / O(J) \cong L_{2}(q)$;

( 5 ) $\operatorname{PS} p(4, q), L_{4}(q)$ or $U_{4}(q)$ with $J / O(J) \cong L_{2}\left(q^{2}\right)$.

Theorem 1.6 (Griess, Solomon [14]). Let $G$ be a minimal unbalanced group with $F^{*}(G)$ quasisimple. Suppose that $a \in \operatorname{Inv}(G)$ and that $J$ is an unbalancing 2-component of $C_{G}($ a) with $J / O(J)$ isomorphic to $\mathrm{He}$ or to a covering group of $L_{3}(4)$. Suppose that $G$ has no unbalancing triple $(b, y, k)$ with $K / Z(K) \cong L_{2}(q)$ for $q \geqq 27$. Then $F^{*}(G) / Z\left(F^{*}(G)\right)$ is isomorphic to $\mathrm{He}$ or $L_{3}(4)$. 
The next result assumes the solution of certain standard form problems. Specifically, the following hypothesis is needed.

Hypothesis S. Let $H$ be a finite group satisfying

(1) $F^{*}(H)$ is simple.

(2) All sections of $H$ satisfy the Unbalanced Group Conjecture.

(3) $H$ has a standard subgroup, $L$, such that one of the following holds:

(a) $L / O(L) \cong \operatorname{PSL}(3,4)$.

(b) $L / Z^{*}(L) \cong \operatorname{PSL}(4,3), \operatorname{PSU}(4,3), \operatorname{PSp}(4,3), \Omega_{7}(3), \Omega^{+}(8,3)$, $\Omega^{-}(8,3)$.

Then $F^{*}(H)$ is a known simple group.

THEOREM 1.7 (Foote [7], Harris [17]). Let $G$ be a finite group with $F^{*}(G)$ simple. Suppose that the following conditions hold:

(1) Proper sections of $G$ satisfy the Unbalanced Group Conjecture.

(2) There exists $t \in \operatorname{Inv}(G)$ and $J$ a 2-component of $C_{G}(t)$ such that $J / O(J) \cong A_{7}$ or $L_{2}(q), q$ odd, and $J$ is maximal in $G$.

(3) Proper sections of $G$ satisfy Hypothesis S.

Then either I or II holds:

I. $J / O(J) \cong L_{2}(q)$ and $F^{*}(G) / F(G)$ is isomorphic to one of

(a) $L_{2}\left(q^{2}\right), t$ a field automorphism;

(b) $L_{3}(q), t$ a graph automorphism;

(c) $U_{3}(q), t$ a field automorphism;

(d) $L_{4}(q), t$ a graph automorphism;

(e) $L_{4}(p), t$ diagonal or graph, $q=p^{2}$;

(f) $U_{4}(q), t$ a graph automorphism;

(g) $U_{4}(p), t$ diagonal or graph, $q=p^{2}$;

(h) $\operatorname{PSp}(4, q), t$ inner or outer (2 classes);

(i ) $\operatorname{PSp}(4, p), t$ outer, $q=p^{2}$;

(j) $\operatorname{Re}^{*}(q), t$ inner;

(k) $L_{2}(16), t$ a field automorphism, $q=5$;

(1) $L_{3}(4), t$ field with $q=7$ or $t$ graph with $q=5$;

(m) $U_{3}(4), t$ outer with $q=5$;

(n) $A_{7}, A_{8}, t$ outer with $q=5,9$ respectively;

(o) $A_{9}, A_{10}, t$ inner with $q=5,9$ respectively;

(p) $J_{1}, t$ inner with $q=5$;

(q) HJ, $t$ inner with $q=5$ or outer with $q=7$;

(r) $J_{3}, t$ outer with $q=17$;

(s) $M_{12}, t$ inner or outer with $q=5$;

(t) HiS, $t$ inner with $q=9$;

(u) $\mathrm{Sp}(4,4)$, $t$ outer, $q=9$;

(v) $\mathrm{GL}(5,2), t$ outer, $q=9$; 
(w) $U_{5}(2), t$ outer, $q=9$;

(x) $\Omega^{-}(8, p), t$ outer, $q=p^{4}$.

II. $J / O(J) \cong A_{7}$ and $F^{*}(G) / F(G)$ is isomorphic to one of

(y) $A_{9}, t$ outer;

(z) $A_{11}, t$ inner;

(zz) He, $t$ outer.

TheOREM 1.8 (Aschbacher [2], Seitz [23], Nah [22], Griess and Solomon [14]). Let $G$ be a finite group satisfying the U.G.-Conjecture. Suppose that $F^{*}(G)$ is simple. Assume that $J$ is a component of $C_{G}(t), t \in \operatorname{Inv}(G)$, with $J / Z(J) \cong L_{3}(4)$ or $J \cong H e$ and that either $C_{G}(J)$ has cyclic Sylow 2-subgroups or $Z(J)$ has even order. Let $J$ be maximal in $G$. Then $F^{*}(G)$ is isomorphic to one of

(a) $L_{3}(16)$.

(b) $\mathrm{He}$.

(c) $O^{\prime} N S$, a sporadic simple group of $O^{\prime} N a n-t y p e$.

In order to state our results we make some further definitions. A maximal unbalancing triple of $G$ is an unbalancing triple $(a, x, J)$ such that if $b \in \operatorname{Inv}\left(C_{G}(a)\right),[J, b] \subseteq O(J)$, and $J$ corresponds to the 2component $L$ of $C_{G}(b)$, then

(1) If $(b, y, L)$ is an unbalancing triple in $G$ for some $y \in C_{G}(a) \cap$ $N_{G}(J)$, then $J$ corresponds isomorphically to $L$, and,

(2) If $S \in \operatorname{Syl}_{2}\left(C_{G}(a) \cap N_{G}(J)\right)$ and $b \in Z(S)$, then $S \in \operatorname{Syl}_{2}\left(C_{G}(b) \cap\right.$ $\left.N_{G}(L)\right)$.

A restricted simple group is one isomorphic to $L_{2}(q), q$ odd, Suz, or one of the groups listed in the conclusions to Theorems 1.7 and 1.8. A group $K$ is of restricted type if $K / Z^{*}(K)$ is a restricted simple group.

Theorem B below is our contribution to the proof of the Unbalanced Group Conjecture. In $\S 4$ we show how Theorem B, all the work previously mentioned, and some additional results recorded below imply the validity of the Unbalanced Group Conjecture under the assumption of Hypothesis S. We formulate this observation as Theorem A.

TheOREM A. Hypothesis S implies the Unbalanced Group Conjecture.

THEOREM B. Let $G$ be a minimal unbalanced group satisfying the following conditions:

(a) $(a, x, J)$ is a maximal unbalancing triple of $G$; 
(b) $J / O(J) \cong L_{2}(q), q$ odd, or $J / O(J) \cong A_{7}$, or $J / Z^{*}(J) \cong L_{3}(4)$;

(c) The solution to the standard component problem for $L_{2}(q)$, $q$ odd, $A_{7}$ and $L_{3}(4)$ in core-free proper sections of $G$ is a central product of quasisimple groups of restricted type.

Then one of the following conclusions holds:

(1) G satisfies the conclusion of the Unbalanced Group Conjecture, or

(2) $J / O(J) \cong L_{2}(q)$ and there exists $b \in \operatorname{Inv}(G)$ and $r$ an odd prime power with $L_{b} / O\left(L_{b}\right) \cong L_{2}\left(r^{2}\right)$ and $\langle b\rangle \in \operatorname{Syl}_{2}\left(C_{G}\left(L_{b} / O\left(L_{b}\right)\right)\right)$, or

(3) (a) If $b \in \operatorname{Inv}\left(C_{G}(a)\right)$ with $[J, b] \subseteq O(J)$, then $J$ corresponds isomorphically to a 2-component $K$ of $C_{G}(b)$; and

(b) If $b$ is as in (a) and $S \in \operatorname{Syl}_{2}\left(C_{G}(a) \cap N_{G}(J)\right)$ with $b \in Z(S)$, then $S \in \operatorname{Syl}_{2}\left(C_{G}(b) \cap N_{G}(K)\right)$.

Corollary C. Let $G$ be a minimal counterexample to the Unbalanced Group Conjecture with $F^{*}(G)$ simple, $a \in \operatorname{Inv}(G)$ and $J a$ 2-component of $C_{G}(a)$ with $J / O(J) \cong A_{7}$. Suppose that there is a 4subgroup $E$ of $C_{G}(a) \cap N_{G}(J)$ with $\Delta=C_{G}(a) \cap\left(\bigcap_{e \in E^{\sharp}} O\left(C_{G}(e)\right)\right)$ and $[J, \Delta]=J$. Then $F^{*}(G) \cong A_{n}$ for some odd $n \geqq 11$.

We remark that Theorem $\mathrm{B}$ and Corollary $\mathrm{C}$ are used in the proof of Theorem 1.6. We use Theorem 1.6 only in $\S 4$ in which we show how Theorem A follows from hypothesis (3) of Theorem 1.7 together with all the theorems in this section.

2. Properties of the restricted simple groups. In this section we collect the properties of the restricted simple groups which we shall need in the proofs of Theorem B and Corollary C.

Proposition 2.1. Assume that $H$ is a group such that

(a) $L \subseteq H \subseteq \operatorname{Aut}(L)$ with $L$ simple of restricted type.

(b) $a \in \operatorname{Inv}(H), J \cong L_{2}(q), A_{7}$ or $L_{3}(4)$, a component of $C_{I I}(a)$, such that $J$ is standard in $H$. Pick $S \in \operatorname{Syl}_{2}\left(C_{H}(a)\right)$ and let $P=C_{S}(J)$, $D=S \cap J$. The following conditions hold:

(1) $P=\langle a\rangle$ except in the following cases:

(i ) $J \cong A_{n}, H \cong A_{n+4}(n \in\{5,6,7\}), P \cong E_{4}$.

(ii) $J \cong A_{5}, H \cong$ Aut $M_{12}, P \cong E_{4}$.

(iii) $J \cong L_{2}\left(q^{2}\right), L \cong L_{4}(q)$ or $U_{4}(q), C_{L}(J) \cong Z_{q+\varepsilon}$ with $\varepsilon= \pm 1$, $q+\varepsilon \equiv 0(\bmod 4)$.

(iv) $J / Z(J) \cong L_{3}(4), L \cong S u z$ or $H e, P \cong E_{4}$.

(v) $J \cong A_{5}, L \cong H J, P \cong E_{4}$.

(2) $P \cap Z(S)=\langle a\rangle$ except when

(i) $J \cong A_{5}, H \cong$ Aut $M_{12}$, or

(ii) $J \cong A_{5}, H \cong H J$. 
Proposition 2.2. Same hypothesis as Proposition 2.1. Let $z \epsilon$ Inv $C_{D}(S)$. Then $a \sim_{H} a z$ except in the following cases:

(1) $L \cong L_{2}\left(q^{2}\right)$ and $H$ does not contain a diagonal automorphism or a field-type automorphism (of order divisible by 4) acting nontrivially on $D /[D, D]$.

(2) Cases (1)(i) and (1)(iii) of Proposition 2.1. In these cases $a \sim z$ in $L$. Moreover if $a_{1} \in \operatorname{Inv} P$, then $a_{1} \sim z$ in $L$.

(3) $J \cong A_{n}, H \cong S_{n+2}(n \in\{5,6,7\})$. Then $a \sim \sigma$ in $H$ where $\sigma$ acts as a transposition on $J$.

(4) $J \cong L_{2}\left(q^{2}\right), H \cong \operatorname{Aut} \operatorname{PSp}(4, q)$. Then $a \sim a \sigma$ for some $\sigma \in$ $C_{L}(a)$ inducing a field automorphism on $J$.

(5) $J \cong Z_{4} \cdot L_{3}(4), L$ of O'Nan-Sims type. Then $a \sim y$ in $L$ for every $y \in \operatorname{Inv}(J)$.

Moreover, if case (1)(v) of Proposition 2.1 holds, then $a_{1} \sim a_{1} z$ in $C_{L}(z)$ for all $a_{1} \in P^{\sharp}$.

We will be confronted with a slightly more general situation arising from the application of $L$-Balance to a group in which the $B(G)$-conjecture holds. If $H$ is such a group and $J$ is a component of $C_{H}(a)$, then $\left\langle J^{L(H)}\right\rangle$ will be quasisimple or a central product of two quasisimple groups permuted by $\langle a\rangle$. Let $a \in \operatorname{Inv}(H)$ and let $J$ be a 2-component of $C_{H}(a)$ with $J / O(J) \cong L_{2}(q), A_{7}$ or $L_{3}(4)$. Take $z, S, P, D$ as above.

Proposition 2.3. Assume that $H$ is a group such that:

(a) The $B(G)$-Conjecture holds in $H$.

(b) $\bar{J}$ is standard and not subnormal in $\bar{H}=H / O_{2^{\prime}, 2}(H)$.

(c) $L(H) / Z^{*}(L(H))$ is a product of restricted groups. The following conclusions hold:

(1) $P=\langle a\rangle\left(P \cap O_{2^{\prime}, 2}(H)\right)$ except as listed in 2.1(1).

(2) $P \cap Z(S)=\langle a\rangle\left(P \cap O_{2^{\prime}, 2}(H)\right)$ except as listed in 2.1(2).

(3) $a \sim_{H} a z$ except in the following cases:

(i) The five cases listed in 2.2 .

(ii) $L / O(L) \cong \mathrm{SL}(2, q) * \mathrm{SL}(2, q)$ with $a \sim a z y, y \in \operatorname{Inv} Z^{*}(L)$.

(iii) $\bar{J} \cong L_{2}(5)$ or $L_{2}(7), \quad L / Z^{*}(L) \cong L_{3}(4)$ with $a \sim a z y, \quad y \in$ Inv $Z *(L)$.

(iv) $\bar{J} \cong L_{2}\left(q^{2}\right), L / Z^{*}(L) \cong L_{4}(q), U_{4}(q)$ or $\Omega^{-}\left(8, q^{1 / 2}\right)$ with $a \sim$ $a z y, y \in \operatorname{Inv} Z^{*}(L)$.

(v) $\bar{J} \cong L_{3}^{\wedge}(4), L / Z^{*}(L) \cong S u z$ with $a \sim a z y, y \in \operatorname{Inv} Z^{*}(L)$.

(4) If $J O(H) / O(H) \cong \mathrm{SL}(2, q)$ and $a \ngtr a z$ in $H$, then $L / Z^{*}(L)$ is isomorphic to one of $\mathrm{SL}(2, q) * \mathrm{SL}(2, q), L_{2}\left(q^{2}\right), A_{7}, A_{8}, A_{9}, A_{10}, L_{3}(4)$ $\operatorname{PSp}(4, \sqrt{q}), L_{4}(\sqrt{q}), U_{4}(\sqrt{q})$ or $\Omega^{-}(8, \sqrt[4]{q})$.

Propositions 2.1-2.3 incorporate all of the facts we need about 
restricted simple groups other than $A_{8}, A_{10}, \operatorname{PSp}(4, q), L_{4}(q), U_{4}(q)$ or $\Omega^{-}(8, q)$, which we treat more fully later.

Proposition 2.1 is easily checked for all of the listed groups. We remark that $H$ may be taken to be $A$ ut $L$ except in the following cases:

(1) $J \cong A_{n}, L=H \cong A_{n+4}$. $J$ is not maximal in $S_{n+4}$.

(2) $J \cong L_{2}(9), L \cong H i S$. $J$ is not maximal in Aut $H i S$.

(3) $\operatorname{PSp}(4, q), L_{4}(q), U_{4}(q)$ discussed below.

Moreover the case $J \cong L_{2}(q), L \cong \operatorname{PSp}(4, q)$ does not occur, since in this case $J$ is not standard in $L$, even though $J$ is maximal in $L$.

If $L / Z(L) \cong A_{n}$, then as $|Z(J)|$ is odd, $|Z(L)|$ is odd. The properties of $A_{n}$ are well-known. If $L / Z(L) \cong L_{12}(16), L_{3}(q)$ or $U_{3}(q)(q$ odd), $U_{3}(4), \operatorname{Sp}(4,4), L_{5}(2), U_{5}(2), \operatorname{Re}(q), J_{1}, J_{3}$, Held's group or a group of O'Nan type, then $|Z(L)|$ is odd. If $L \cong J_{1}$ or $\operatorname{Re}(q)$ or of O'Nan type, then $a \in L$ and $L$ has one class of involutions. In the other cases $L a$ has one class of involutions. So Propositions 2.2 and 2.3 hold for all of these groups.

If $L / Z(L) \cong H i S$, we refer the reader to [15] for the desired properties of $L$.

If $L / Z(L) \cong L_{3}(4)$, then Proposition 2.3 only makes an assertion about fusion modulo $Z(L)$. As each coset of $\operatorname{Inn} L$ in Aut $L$ has at most one class of involutions, this is clear.

We now treat $M_{12}, H J$ and $S u z$.

Proposition 2.4. (a) Suppose that $L / Z(L) \cong M_{12}$. Then $J \cong A_{5}$ and $C_{\text {Aut } L}(J)=P \cong E_{4}$ with $|P \cap \operatorname{Inn} L|=2$. $\quad N_{\text {Aut } L}(J)=(P \times J)\langle b\rangle$ with $\left|C_{P}(b)\right|=2$. If $a \in C_{P}(b)^{\sharp}$, then $a \sim a z$ in $C_{L}(z)$.

(b) Suppose that $L / Z(L) \cong H J$. If $a \notin \operatorname{Inn} L$, then $J \cong L_{2}(7)$, $C_{\text {Aut } L}(J)=\langle a\rangle$ and $a \sim a z$ in $\langle L, a\rangle$. If $a \in L$, then $J \cong A_{5}$ and $C_{\text {Aut } L}(J)=P \cong E_{4}$. If $a_{1} \in P^{\#}$, then $a \sim a_{1}$ and $a \sim a z$ in $C_{L}(z)$.

(c) Suppose that $L / Z(L) \cong S u z$ and $J / Z(J) \cong L_{3}(4)$. Then $Z(L) \cong$ $Z(J)$. Also $C_{\text {Aut } L}(J)=P \cong E_{4}$ and $a_{1} \sim a_{1} z$ in $L$ for all $a_{1} \in P^{\sharp}$.

Proof. Let $\bar{L}=L / Z(L)$ and identify $\bar{L}$ with $\operatorname{Inn} L$.

(a) Aut $L$ contains a 4-group, $\bar{P}$, with $C_{\text {aut } L}(\bar{P})=\bar{P} \times \bar{J}$ and $N_{{\text {Au }{ }_{L}}_{L}}(\bar{P})=(\bar{P} \times \bar{J})\langle\bar{b}\rangle$ with $\langle\bar{P}, \bar{b}\rangle \cong D_{8},\langle\bar{J}, \bar{b}\rangle \cong S_{5}$. As $(\bar{P} \times \bar{D})\langle\bar{b}\rangle \notin$ $\operatorname{Syl}_{2}($ Aut $L)$ and $\bar{D}^{\sharp}=\bar{z}^{\mathrm{Aut} L} \cap(\bar{P} \times \bar{D})$, there exists $\bar{y} \in N_{\bar{L}}(\bar{P} \times \bar{D}) \cap N_{\bar{L}}(\bar{D})$ with $\bar{P} \cap \bar{P}^{\bar{y}}=1$. Thus if $\langle\bar{a}\rangle=C_{\bar{P}}(\bar{b})$, then $\bar{a}^{\bar{y}}=\bar{z}$ and $\bar{z}^{\bar{y}}=\bar{z}$. If $\bar{a}_{1} \in \bar{P}-\langle\bar{a}\rangle$, then $\bar{a}_{1}^{\bar{y}_{1}}=\bar{a}_{1} \bar{z}_{1}$ for some $\bar{z}_{1} \in \bar{D}-\langle\bar{z}\rangle, \bar{y}_{1} \in N_{\bar{L}}(\bar{D})$. Thus $\bar{a}_{1} \sim \bar{a}_{1} \bar{z}$ in $N_{\bar{L}}(D)$. Suppose that $\mid Z(L)_{1}=2$. Now $\bar{b}, \bar{a}$ and $\bar{a} \bar{b}$ are noncentral involutions of $\bar{L}$. So $\langle a, b\rangle \cong Q_{8}$ and $a \sim a z$ in $C_{L}(z)$. Also $\bar{a}_{1} \sim \bar{a} \bar{a}_{1}$. So $a_{1}$ and $a a_{1}$ have the same order in $L$. Thus $a_{1}$ inverts $a$ and $a_{1} \sim a_{1} z$ in $N_{L}(D)$.

(b) Suppose that $a \notin \operatorname{Inn} L$. Then $L a$ has only one class of in- 
volutions. Thus we may assume that $Z(L) \neq 1$. Now $C_{\bar{L}}(\bar{a})=\bar{J}\langle\bar{s}\rangle$ with $\bar{s}$ a noncentral involution. Thus $s$ has order 4 in $L$. As $\bar{a} \sim \bar{a} \bar{s}, a$ and $a s$ have the same order in $L$. So $a$ inverts $s$. Thus $a \sim a z$ in $\langle L, a\rangle$.

Now suppose that $a \in \operatorname{Inn} K$. Then $\bar{J} \cong A_{5}$ and $C_{\bar{L}}(\bar{J})=\bar{P} \cong E_{4}$. As $\bar{a} \sim \bar{a}_{1}$ for all $a_{1} \in \bar{P}^{\#}, \bar{P} \times \bar{J}=C_{\bar{L}}\left(\bar{a}_{1}\right)$ for all $\bar{a}_{1} \in \bar{P}^{\#}$. Thus there exists $\bar{r} \in C_{\bar{L}}(\bar{J}) \cap N_{\bar{L}}(\bar{P})$ permuting the elements of $\bar{P}^{\#}$ and $\langle\bar{P}, \bar{r}\rangle \times$ $\bar{J}=N_{\bar{L}}(\bar{P})$. Let $\bar{E} \in \operatorname{Syl}_{2}(\bar{P} \times \bar{J})$. Then $N_{\bar{L}}(\bar{P}) \cap N_{\bar{L}}(\bar{E})$ has three orbits on $\bar{E}^{\#}$ with lengths 3 , 3, 9. As $\bar{a}^{\bar{L}} \cap \bar{D}=\varnothing$ and $\bar{E} \in \operatorname{Syl}_{2}(\bar{L}),\left|\bar{a}^{\bar{L}} \cap \bar{E}\right|=$ 12. Thus $N_{\bar{L}}(\bar{E}) \cap C_{\bar{L}}(\bar{D}) / \bar{E} \cong A_{4}$ and $N_{\bar{L}}(\bar{E}) \cap C_{\bar{L}}(\bar{D})=O^{2}\left(N_{\bar{L}}(\bar{E}) \cap C_{\bar{L}}(\bar{D})\right.$ ). Thus $\bar{a}_{1} \sim \bar{a}_{1} \bar{z}_{1}$ in $C_{\bar{L}}(\bar{D}) \cap N_{\bar{L}}(\bar{E})$ for all $\bar{a}_{1} \in \bar{P}^{\sharp}, \bar{z}_{1} \in \bar{D}$. If $Z(L) \neq 1$, then $P \cong Q_{8}$ and $N_{L}(E) \cap C_{L}(D)$ is the full inverse image in $L$ of $N_{\bar{L}}(\bar{E}) \cap C_{\bar{L}}(\bar{D})$. Thus $a_{1} \sim a_{1} z_{1}$ in $C_{L}(D)$ for all $a_{1} \in P-Z(P), z_{1} \in D$.

(c) Let $S \hat{u} z$ be the six-fold cover of $S u z$ and let $\rho$ be a 12dimensional complex representation of $S \hat{u} z$. We wish to show that the inverse image, $\hat{J}$, of $J$ in $S \hat{u} z$ is a six-fold cover of $L_{3}(4)$. Now $C_{S u z}(J)=P \cong E_{4}$ and $N_{S u z}(P) \cong\left(A_{4} \times L_{3}(4)\right) \cdot Z_{2}$.

Also $\hat{P} \cong Q_{8}$ and $Z(\hat{P})$ acts as $-I$. So $\hat{P}$ must act on a sum of six isomorphic 2-dimensional modules. Then $C_{\rho(S \breve{u} z)}(\rho(\hat{P}))$ is isomorphic to a subgroup of $\mathrm{GL}(6, C)$. Then, by Lindsey [18], $\hat{J}$ is a six-fold cover of $L_{3}(4)$. Thus $Z(K) \subseteq Z(J)$. If $\bar{S} \in \operatorname{Syl}_{2}\left(N_{\bar{L}}(\bar{P})\right)$, then $\bar{S} \subset \bar{T} \in$ $\operatorname{Syl}_{2}(\bar{L})$ and $\bar{P} \cap \bar{P}^{\bar{y}}=1$ for $\bar{y} \in N_{\bar{T}}(\bar{S})-\bar{S}$. As $a^{\bar{T}} \cap \bar{J}=\varnothing$ and $z^{\bar{J}}=$ Inv $\bar{J}, \bar{a} \sim \bar{a} \bar{z}$ in $\bar{L}$. As $\hat{P} \cong Q_{8}$ in $S \hat{u} z$, we see as before that $a \sim a z$ in $L$.

Proposition 2.5. Let $K$ be isomorphic to $A_{8}$ or to $\operatorname{PSp}(4, q), q$ odd. Let $N=S_{8}$ in the former case and let $N$ be the extension of $\operatorname{PSp}(4, q)$ by a diagonal automorphism in the latter case, i.e., $N \cong$ $\mathrm{SO}(5, q)$ in the latter case.

(a) If $f \in$ Aut $K-N$ of order 2 , then $K \cong \operatorname{PSp}\left(4, q_{1}^{2}\right), f$ is a field automorphism and $L\left(C_{K}(f)\right) \cong \operatorname{PSp}\left(4, q_{1}\right)$.

(b) $N$ has four classes of involutions, two in $K$ and two in $N-K . \quad N$ has exactly one 2-central class of involutions.

(c) If $s$ is a 2-central involution of $N$, then $O^{2}\left(C_{N}(s)\right) \cong$ $\mathrm{SL}(2, q) * \mathrm{SL}(2, q)$ if $K \cong \operatorname{PSp}(4, q)$ and $C_{N}(s)$ is solvable if $K \cong A_{8}$.

(d) If $a \in K$ is a non-2-central involution, and $\varepsilon= \pm 1$ so that $4 \mid q+\varepsilon$, then

$$
C_{N}(a) \cong \mathrm{PGL}(2, q) \times D_{2(q+\varepsilon)}
$$

with $q=3$ when $K \cong A_{8}$.

Also $C_{K}(\alpha)=(L \times F)\langle\alpha\rangle$ with $L \cong L_{2}(q),\langle L, \alpha\rangle \cong \operatorname{PGL}(2, q), F \cong$ $D_{q+\varepsilon}$ and $\langle F, \alpha\rangle \cong D_{2(q+\varepsilon)}$.

If $z \in \operatorname{Inv} L$, then $a \sim z$ in $K$.

(e) If $e \in \operatorname{Inv}(N-K)$, then $O^{2}\left(C_{N}(e)\right)$ is isomorphic to $L_{2}(q)$ or $L_{2}\left(q^{2}\right)$, with $q=3$ when $K=A_{8}$. If $L\left(C_{N}(e)\right)$ is a maximal component 
in $N$, then $L\left(C_{N}(e)\right) \cong L_{2}\left(q^{2}\right)$. If $O^{2}\left(C_{N}(e)\right) \cong L_{2}(q)$, then $C_{K}\left(\left\langle e, O^{2}\left(C_{N}(e)\right)\right\rangle\right) \cong$ $D_{q-\varepsilon}$ with $\varepsilon$ as in (d).

(f) No proper covering of $K$ has an involutory automorphism whose centralizer has an $L_{2}(q)$ component.

Proof. The properties of $S_{8}$ are well-known. The conjugacy of all involutory field automorphism is a well-known consequence of Lang's theorem. Properties (b)-(e) may be found in [10], [11] or deduced directly by matrix calculations in $N \cong \mathrm{SO}(5, q)$. Elements of $\Omega(5, q)$ with exactly two eigenvalues -1 are known to lift to elements of order 4 in $\operatorname{Spin}(5, q)$. (See [24, Lemma 3.1].) If $M$ is an $L_{2}(q)$ component in $K$, then the involutions of $M$ have exactly two eigenvalues -1 , whence $\widehat{M} \cong \operatorname{SL}(2, q)$ in $\operatorname{Spin}(5, q)$.

Proposition 2.6. Same hypotheses as Proposition 2.5. Take $a \in \operatorname{Inv} K$ with a non-2-central and take $e \in \operatorname{Inv}(N-K)$ with $L=$ $L\left(C_{K}(e)\right) \cong L_{2}\left(q^{2}\right)$. Take $S \in \operatorname{Syl}_{2}\left(C_{N}(a)\right), \quad S \subset R \in \operatorname{Syl}_{2}(N)$. Let $P=$ $S \cap C_{N}(J), D=S \cap J, S_{0}=S \cap K, P_{0}=P \cap K, R_{0}=R \cap K$.

(a) $\left[R_{0}, R_{0}\right]$ is nonabelian; $R=\Omega_{1}(R), R_{0}=\Omega_{1}\left(R_{0}\right)$.

(b) When $K=\operatorname{PSp}(4, q)$, all elements of $\operatorname{Inv}\left(P_{0}-\langle a\rangle\right)$ are 2central.

(c) $|R: S|=\left|R_{0}: S_{0}\right|=2$. For any $r \in \operatorname{Inv}(R-S), a^{r}=z, z^{r}=a$.

(d) $d_{0}$ contains $E \cong E_{16}$ and all such $E$ are conjugate in $N$. $N_{N}(E) / C_{N}(E) \cong S_{5}$ or $S_{3}$ 乙 $Z_{2}$, according as $K=\operatorname{PSp}(4, q)$ or $A_{8}$.

(e ) Pick $Q \in \operatorname{Syl}_{2}\left(C_{N}(e)\right)$ with $Q \subseteq T \in \operatorname{Syl}_{2}(N)$ and $z \in \operatorname{Inv}\left(C_{L}(Q)\right)$. Then $C_{K}(e)=L\langle\tau\rangle$ with $\tau$ a non-2-central involution in $K$, $\tau$ acting as a field automorphism on L. Further $e^{g}=e^{\tau}$ for some $g \in N_{T}(Q)-Q$

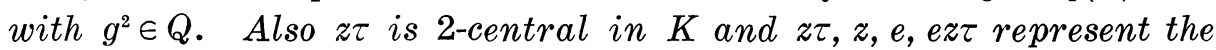
$N$-classes of involutions.

Proof. We refer the reader to [10], [11] and direct matrix calculation.

Proposition 2.7. Let $K=\operatorname{PSL}(4, q), q$ odd. Let $N$ be a normal complement in Aut $K$ to the cyclic group of field automorphisms.

(a) If $N \neq O^{2^{\prime}}$ (Aut $K$ ), then (Aut $K$ ) $-N$ has two classes of involutions whose fixed points on $K$ contain $\operatorname{PSL}\left(4, q^{1 / 2}\right)$ and $\operatorname{PSU}\left(4, q^{1 / 2}\right)$ respectively.

(b) If $q \equiv 3(\bmod 4)$, then Aut $K / \operatorname{Inn} K$ is abelian.

(c) $N$ has six classes of involutions.

(d) If $s$ is a 2-central involution in $N$, then $O^{2}\left(C_{N}(s)\right) \cong$ $\mathrm{SL}(2, q) * \mathrm{SL}(2, q)$.

(e) $N$ has two classes of diagonal involutions with representatives $a$ and $a_{1}$. $L\left(C_{N}(a)\right) \cong L_{2}\left(q^{2}\right)$ and $L\left(C_{N}\left(a_{1}\right)\right) \cong L_{3}(q)$. Also $a$ is 
inner if and only if $q \equiv 3(\bmod 4) . \quad C_{\left\langle_{K}, a\right\rangle}(a)=(\langle t\rangle \times J)\langle\sigma\rangle$ with $\langle t\rangle \cong \boldsymbol{Z}_{q+1}, \quad J \cong L_{2}\left(q^{2}\right), \sigma$ inverting $\langle t\rangle$ and. inducing a field automorphism on $J$. Also if $z \in \operatorname{Inv} J$ and $a \in K$, then $a \sim z$ in $K$. If $a \notin K$, then $a \sim a z$ in $\langle K, a\rangle$.

(f) $N$ has three classes of graph automorphisms of order 2 with representatives $b, c, d . \quad L\left(C_{N}(b)\right) \cong \operatorname{PSp}(4, q), L\left(C_{N}(c)\right) \cong L_{2}\left(q^{2}\right)$ and $O^{2}\left(C_{N}(d)\right) \cong L_{2}(q) \times L_{2}(q)$. Let $M$ be a subgroup of $N$ containing $\langle K, a\rangle$ such that $L\left(C_{H}(a)\right)$ is maximal in $M$. Then $b \notin M$ and $\langle t\rangle=C_{M}(J)$. Let $M_{1}$ be a subgroup of $N$ containing $\langle K, c\rangle$ such that $L\left(C_{K}(c)\right)$ is maximal in $M_{1}$. Then $b \notin M_{1}$ and $\langle c\rangle=C_{M_{1}}\left(L\left(C_{K}(c)\right)\right)$. Every involution of $K c$ is $K$-conjugate to $c$. In particular, if $z \in \operatorname{Inv}\left(L\left(C_{K}(c)\right)\right)$, then $c \sim c z$ in $\langle K, c\rangle$.

(g) The full coverging group $\hat{K}$ of $K$ does not admit an involutory automorphism whose centralizer has an $L_{2}(q)$ component. Also $Z(\hat{K})$ is cyclic of order 2 or 4 .

Proof. Most of these facts may be found in [6] and [20], [21]. The rest follow by direct matrix calculation. It is helpful to recall that $L_{4}(q) \cong P \Omega^{+}(6, q)$. The existence of precisely three classes of graph automorphisms may be found in [6]. Both $b$ and $d$ lie in the coset of $P \Omega^{+}(6, q)$ by a diagonal matrix with precisely one eigenvalue -1. It follows that all involutions in $K c$ are $K$-conjugate to $c$. Fact (g) follows as in Proposition 2.5 from the properties of $\operatorname{Spin}(6, q)$.

Proposition 2.8. (1) Let $K=\operatorname{PSU}(4, q), q$ odd and $N=$ Aut $K$. The assertions of Proposition 2.7 remain true after replacing (b) by:

$\left(\mathrm{b}^{\prime}\right)$ If $q \equiv 1(\bmod 4)$, then Aut $K / \operatorname{Inn} K$ is abelian. And replacing (e) by:

( $\left.\mathrm{e}^{\prime}\right) \quad N$ has two classes of diagonal involutions with representatives a and $a_{1}$. $L\left(C_{N}(a)\right) \cong L_{2}\left(q^{2}\right)$ and $L\left(C_{N}\left(a_{1}\right)\right) \cong U_{3}(q)$. Also a is inner if and only if $\left.q \equiv 1(\bmod 4) . \quad C_{\left\langle_{K}, a\right.}\right\rangle(a)=(\langle t) \times J)\langle\sigma\rangle$ with $\langle t\rangle \cong \boldsymbol{Z}_{q-1}, \quad J \cong L_{2}\left(q^{2}\right), \sigma$ inverting $\langle t\rangle$ and inducing a field automorphism on $J$. If $z \in \operatorname{Inv} J$ and $a \in K$, then $a \sim z$ in $K$. If $a \notin K$, then $a \sim a z$ in $\langle K, a\rangle$.

(2) Let $K=A_{10}, N=S_{10} . \quad N$ has one class of involutions with representative a satisfying $L\left(C_{N}(a)\right) \cong A_{6} . \quad C_{N}(a) \cong S_{6} \times D_{8} . \quad C_{K}(a)=$ $(J \times P)\langle\sigma\rangle$ with $J \cong A_{6}, P \cong E_{4}, J\langle\sigma\rangle \cong S_{6}, P\langle\sigma\rangle \cong D_{8}$. $N$ has one class of involutions with representative $b$ satisfying $L\left(C_{N}(b)\right) \cong A_{8}$. $C_{K}(b) \cong S_{8}$ and $b \notin K . \quad \hat{A}_{10}$ does not admit an involutory automorphism whose centralizer has a subnormal subgroup isomorphic to $A_{4}$ or $A_{6}$.

Proof. (1) is handled like Proposition 2.7. The assertions about $S_{10}$ are trivial. An involution $r$ in an $A_{4}$ or $A_{6}$ subnormal in $C_{G}(s)$ for some $s \in \operatorname{Inv} S_{10}$ has the property that $r$ is a product of two 
transpositions. Then $r$ lifts to an element of order 4 in $\hat{A}_{10}$.

Proposition 2.9. Let $K=A_{10}, L_{4}(q)$ or $U_{4}(q)$ with notation as in 2.7 and 2.8. Suppose that $a \in K$, i.e., $K=A_{10}$ or $K=L_{4}(q), q \equiv 3$ $(\bmod 4)$ or $K=U_{4}(q), q \equiv 1(\bmod 4)$. Let $S \in \operatorname{Syl}_{2}\left(C_{N}(a)\right), S \subset R \in \operatorname{Syl}_{2}(N)$. Let $R_{0}=R \cap K, D=S \cap J, P=C_{S}(J)$. Then

(1) $\left[R_{0}, R_{0}\right]$ is nonabelian.

(2) There exists $t \in N_{R_{0}}(S)-S$ with $t^{2} \in S_{0}$ and $D D^{t}=D \times D^{t}=$ $S_{0}$.

(3) $\left|D^{t}: D^{t} \cap P\right|=2$ and $d \in D^{t}-P$ acts as a field automorphism or transposition on $J$.

(4.) $\left(D^{t} \cap P\right)\langle b\rangle$ is dihedral with center $\langle a\rangle$.

Proof. Direct calculation. Note that $R_{0} \cong D_{2^{n}} 2 Z_{2}$ with $2^{n} \| q^{2}-1$. Here $q=3$ if $K=A_{10}$.

Proposition 2.10. Let $K$ be a finite quasisimple group with $\bar{K}=K / Z(K) \cong \Omega_{8}^{-}(q)$.

(1) $Z(K)$ is cyclic of order 2 or 4.

(2) There is a unique class of involutory automorphisms with representative a such that $J=L\left(C_{K}(a)\right) \cong L_{2}\left(q^{4}\right)$. If $b \in \operatorname{Inv}$ Aut $K$ with $C_{K}(b)$ having a maximal component isomorphic to $L_{2}(r)$ for any $r$, then $b \in a^{K}$.

(3) $\operatorname{Inv}(\bar{K} a)=a^{K}$. In particular, if $z \in \operatorname{Inv}(J)$, then $a \sim a z y$ in $\langle K, a\rangle$ for some $y \in Z(K)$. Also $\langle a\rangle \in \operatorname{Syl}_{2}\left(C_{\text {Aut }_{K}}(J)\right)$.

(4) There is no involution $\bar{\alpha}$ in $\bar{K}$ with $O^{2}\left(C_{\bar{K}}(\bar{\alpha})\right)$ of 2-rank 1.

Proof. We refer the reader to [6].

Proposition 2.11. Let $G$ be a finite group of sectional 2-rank 4 with $F^{*}(G)$ simple. Suppose that $G$ has an involution a with $L\left(C_{G}(a)\right) \cong$ $L_{2}\left(q^{2}\right)$ for some odd $q \geqq 3$. Suppose also that $G \cong G_{1}$ of index 2 and $b \in \operatorname{Inv} C_{G_{1}}(a)$ with either $L\left(C_{G}(b)\right) \cong \operatorname{PSp}(4, q)$ or $q=3$ and $L\left(C_{G}(b)\right) \cong$ $A_{8}$. Then $F^{*}(G) \cong L_{4}(q), U_{4}(q)$ or $A_{10}$.

Proof. We may check the list of conclusions to the Main Theorem of [12].

Proposition 2.12. Let $K \cong \operatorname{PSp}(4, q), L_{4}(q), U_{4}(q)$ or $\Omega^{-}(8, q), q$ odd. Suppose that $a$ is an involutory automorphism of $K$ with $L\left(C_{K}(a)\right)=1$. Then $K \cong \operatorname{PSp}(4,3), L_{4}(3)$ or $U_{4}(3)$. Moreover $C_{K}(a)$ involves $A_{4}$.

Proof. The information for $\operatorname{PSp}(4, q), L_{4}(q)$ and $U_{4}(q)$ may be 
read off from Propositions 2.5, 2.7 and 2.8. For $\Omega^{-}(8, q)$ one may check the information in [6] or compute directly in $O^{-}(8, q)$.

3. Preliminary results. We are principally concerned here with properties of 2-components. We begin with a reduction of the Unbalanced Group Conjecture.

Lemma 3.1. Suppose that every proper section of $G$ satisfies the Unbalanced Group Conjecture, then either $G$ satisfies the Unbalanced Group Conjecture or $F^{*}(G)$ is simple.

Proof. Suppose $G$ does not satisfy the U.G.-Conjecture. Take $x \in \operatorname{Inv}(G)$ with $D=O\left(C_{G}(x)\right) \nsubseteq O(G)$. Minimality implies $O(G)=1$, and by [26, Lemma 2.5] $G$ has an (unbalanced) component $L$ such that $L=[L, D]=[L, x]$. By minimality again $G=\langle L, D, x\rangle$ whence $L=F^{*}(G)$. It remains only to show $Z(L)=1$. Suppose $Q=Z(L) \neq 1$. $Q$ is a 2-group, so $C_{Q}(x) \leqq O_{2}\left(C_{G}(x)\right)$ implies $\left[C_{Q}(x), D\right]=1$. By Thompson's $A \times B$ lemma, $[Q, D]=1$. Now $Z(G)=C_{Q}(x) \neq 1$, and one sees easily that $G / Z(G)$ is an unbalanced group. (Note that $x \notin Z(G)$ else $D \subseteq O(G)=1$.) Since the Unbalanced Group Conjecture holds for $G / Z(G)$, and $L / Z(G)=L(G / Z(G))$, we conclude that $L$ is simple.

Next we wish to develop a particular property of 2-components. Suppose $J$ is a 2-component of $C_{G}(a), a \in \operatorname{Inv}(G)$, with $a \in Z(S), S \in$ $\operatorname{Syl}_{2}\left(N_{G}(J)\right)$. Let $P=C_{S}(J)$; for any 2-group $B \cong P, B$ has a conjugate $B^{x} \subseteq P$ such that $Q=N_{S}\left(B^{x}\right) \in \operatorname{Syl}_{2}\left(N_{G}\left(B^{x}\right) \cap N_{G}(J)\right)$. If $G$ satisfies the $B(G)$-Conjecture, then $J_{B^{x}}=J$ centralizes $O\left(C_{G}\left(B^{x}\right)\right)$, and $Q \in$ $\operatorname{Syl}_{2}\left(N_{G}\left(B^{x}\right) \cap N_{G}\left(J_{B^{x}} O\left(C_{G}\left(B^{x}\right)\right)\right)\right)$. Lemma 3.3 shows that this result holds if $G$ is a minimal unbalanced group. The next lemma is used in the proof of Lemma 3.3.

LeMma 3.2. Suppose all 2-local subgroups of G satisfy the $B(G)$ Conjecture. Let $J$ be a 2-component of $C_{G}(Q), Q$ a 2-subgroup of $G$, and let $P$ and $R$ be 2-subgroups with $P \subseteq R \subseteq C_{G}(Q) \cap C_{G}(J / O(J))$. Define $H=C_{G}(P), \bar{H}=H / O(H)$. The following conditions hold:

(i) $\overline{J_{P}}=\overline{J_{R}}$;

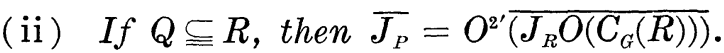

Proof. $[J, P] \subseteq O(J)$ implies $J_{P}=O^{2^{\prime}}\left(C_{J}(P)\right)$. Likewise $J_{R}=$ $O^{2^{\prime}}\left(C_{J}(R)\right)$; and it follows that $J_{R} \leqq J_{P}$ and $J_{R}$ covers $J_{P} / O\left(J_{P}\right)$. As $\overline{J_{P}}$ is quasisimple by the $B(G)$-Conjecture, $\overline{J_{R}}$ covers $\overline{J_{P}} / Z\left(\overline{J_{P}}\right)$. Since $\overline{J_{P}}$ is perfect, $\overline{J_{R}}=\overline{J_{P}}$ and (i) holds.

We prove (ii). By $L$-Balance $\overline{J_{P}}=\overline{J_{R}}$ is a component of $C_{\bar{H}}(\bar{Q})$. Let $F=O\left(C_{G}(R)\right)$. As $P Q \subseteq R, F \subseteq C_{H}(Q) . \quad\left[J_{R}, F\right] \subseteq\left[C_{G}(R), F\right] \subseteq F$ 
implies $\left[\overline{J_{R}}, \bar{F}\right] \leqq \bar{F}$ in $\bar{H}$. Since $\bar{F}$ permutes the components of $C_{\bar{H}}(\bar{Q}), \bar{F}$ normalizes $\overline{J_{R}}$. Thus $O^{2} \overline{\left(J_{R} F\right)}=\overline{J_{R}}=\overline{J_{P}}$.

Lemma 3.3. Let $G$ be a group with all 2-local subgroups satisfying the $B(G)$-Conjecture. Suppose $J$ is a 2-component of $C_{G}(a), a \epsilon$ Inv $(G)$, and $S \in \operatorname{Syl}_{2}\left(C_{G}(a) \cap N_{G}(J)\right)$. Let $P=C_{S}(J / O(J))$, and assume

For every $b \in \operatorname{Inv}\left(C_{P}(S)\right)$,

$S \in \operatorname{Syl}_{2}\left(C_{G}(b) \cap N_{G}\left(J_{b} O\left(C_{G}(b)\right)\right)\right)$.

Then for every $B \subseteq P$ there exists $x \in G$ such that

(i) $x=x_{1} \cdots x_{t}$ and $\left\langle\left(B^{x_{1} \cdots x_{i-1}}\right)^{\left\langle x_{i}\right\rangle}\right\rangle \leqq P, 1 \leqq i \leqq t$;

(ii) $N_{S}\left(B^{x_{1} \cdots x_{i-1}}\right)^{x_{i}} \leqq S, 1 \leqq i \leqq t$;

(iii) $J_{B^{x}} O\left(C_{G}\left(B^{x}\right)\right)=\left[J_{B} O\left(C_{G}(B)\right)\right]^{x}$;

(iv) $N_{S}\left(B^{x}\right) \in \operatorname{Syl}_{2}\left(N_{G}\left(B^{x}\right) \cap N_{G}\left(J_{B^{x}} O\left(C_{G}\left(B^{x}\right)\right)\right)\right)$.

Proof. First we show that (i) implies (iii). By induction on $t$ we may let $x=y x_{t}$ and suppose $J_{B^{y}} O\left(C_{G}\left(B^{y}\right)\right)=\left[J_{B} O\left(C_{G}(B)\right)\right]^{y}$. Let $x_{t}=z, E=B^{y}$ and $T=\left\langle E^{\langle z\rangle}\right\rangle$; by (i) $T \subseteq P$. By Lemma 3.2 (i) $J_{E} O\left(C_{G}(E)\right)=J_{T} O\left(C_{G}(E)\right)$ and likewise $J_{E^{z}} O\left(C_{G}\left(E^{z}\right)\right)=J_{T} O\left(C_{G}\left(E^{z}\right)\right)$. Now

$$
\begin{aligned}
{\left[J_{B} O\left(C_{G}(B)\right)\right]^{x} } & =\left[J_{E} O\left(C_{G}(E)\right)\right]^{z}=\left[J_{T} O\left(C_{G}(E)\right)\right]^{z}=J_{T^{z}} O\left(C_{G}\left(E^{z}\right)\right) \\
& =J_{E^{z}} O\left(C_{G}\left(E^{z}\right)\right)=J_{B^{x}} O\left(C_{G}\left(B^{x}\right)\right) .
\end{aligned}
$$

We will now use induction on $|P: B|$ to prove Lemma 3.3 (i), (ii), (iv). Let $X=J_{B} O\left(C_{G}(B)\right)$ and suppose $Q=N_{S}(B) \notin \operatorname{Syl}_{2}\left(N_{G}(B) \cap N_{G}(X)\right)$. Pick a 2-group $Q_{1}$ with $Q \leqq Q_{1}, Q \neq Q_{1}$, and $Q_{1} \subseteq N_{G}(B) \cap N_{G}(X)$. If $B=P$, then $Q=S$ and $Q_{1}$ centralizes some $b \in \operatorname{Inv}\left(C_{B}(S)\right) . Q_{1}$ normalizes $O^{2 \prime}(X) O\left(C_{G}(b)\right)=J_{b} O\left(C_{G}(b)\right)$ by Lemma 3.2 (ii) contradicting (**). Thus $B \neq P$. Let $T=Q \cap P$; we have $B \subset T$. By induction we may assume $N_{S}(T) \in \operatorname{Syl}_{2}\left(N_{G}(T) \cap N_{G}\left(J_{T} O\left(C_{G}(T)\right)\right)\right), \quad Q_{1}$ normalizes $T=$ $C_{Q}(X / O(X))$ and $C_{X}(T)$. By Lemma 3.2 (i) $X=J_{T} O\left(C_{G}(B)\right)$ whence $C_{X}(T)=J_{T}\left(O\left(C_{G}(B)\right) \cap C_{G}(T)\right)$. It follows that $Q_{1}$ normalizes $J_{T} O\left(C_{G}(T)\right)=$ $C_{X}(T) O\left(C_{G}(T)\right)$. Thus $Q_{1} \subseteq N_{G}(T) \cap N_{G}\left(J_{T} O\left(C_{G}(T)\right)\right)$ and these exists $x \in N_{G}(T) \cap N_{G}\left(J_{T} O\left(C_{G}(T)\right)\right)$ such that $Q_{1}^{x} \subseteq N_{S}(T)$. Clearly $\left\langle B^{\langle x\rangle}\right\rangle \leqq T \leqq P$ and $Q_{1}^{x} \cong N_{S}\left(B^{x}\right) \cong N_{G}\left(B^{x}\right) \cap N_{G}\left(J_{B^{x}} O\left(C_{G}\left(B^{x}\right)\right)\right)$. As $\left|Q_{1}\right|>|Q|$, repeating our argument a finite number of times gives (iv).

Lemma 3.4. Let $J$ be a 2-component of $C_{G}(a), a \in \operatorname{Inv}(G)$. Suppose $m(J) \geqq 2$ and $b \in \operatorname{Inv}\left(C_{G}(a)\right)$ with $[J, b] \subseteq O(J)$. Pick $S \in \operatorname{Syl}_{2}\left(C_{G}(a) \cap\right.$ $\left.N_{G}(J)\right)$ with $\langle a, b\rangle \leqq S$, and let $P=C_{S}(J / O(J))$. Let $J$ correspond to the 2-component $K$ of $C_{G}(b)$. Either $J$ corresponds isomorphically to $K$ or there exists $e \in \operatorname{Inv}(P)$ such that the following conditions hold:

(1) $C_{S}(b) \subseteq C_{S}(e)$

(2) If $J$ corresponds to the 2-component $L$ of $C_{G}(e)$, then 
$|L: O(L)|>|J: O(J)| \operatorname{or} m\left(C_{G}(e) \cap C_{G}(L / O(L))\right)>m\left(C_{G}(a) \cap C_{G}(J(O(J)))\right)$.

Proof. Suppose $J$ does not correspond isomorphically to $K$. Pick $e \in \operatorname{Inv}(P)$ so that $C_{S}(b) \subseteq C_{S}(e)=Q$ and $Q$ is maximal with respect to inclusion among choices of $e$ for which $J$ does not correspond isomorphically to a 2-component of $C_{G}(e)$. By $L$-Balance either $L \neq L^{a}$ or $|L: O(L)|>|J: O(J)|$. We may assume $L \neq L^{a}$ and $|L: O(L)|=$ $|J: O(J)|$. Define $M=C_{G}(e), \bar{M}=M / O(M) . \quad \bar{L}=\bar{J}_{e}$ implies $\bar{L} \bar{L}^{\bar{a}}=$ $\bar{L} \times \bar{L}^{\bar{u}}$. By [13, Lemma 2.14], $\bar{J}_{e}=\left\{\bar{y} \bar{y}^{\bar{u}} \mid \bar{y} \in \bar{L}\right\}$ is isomorphic to $\bar{L}$. As $T=Q \cap P$ centralizes $J_{e} / O\left(J_{e}\right), T$ acts on $\bar{L} \bar{L}^{\bar{a}}$ and centralizes $\bar{J}_{e}$. It follows that $T=\langle a\rangle \times R, R=C_{T}\left\langle\bar{L} \bar{L}^{\bar{a}}\right\rangle$. Pick $F \in \operatorname{Syl}_{2}(L)$ so that $\langle F, Q\rangle$ is a 2-group. As $\bar{L} \cap \bar{L}^{\bar{a}}=1, \bar{F} \bar{F}^{\bar{u}}=\bar{F} \times \bar{F}^{\bar{c}}$ and $C_{\bar{F}}(\bar{a})=1$ implies $\bar{F} \cap \bar{R}=1$. It follows that $m\left(C_{G}(e) \cap C_{G}(\bar{L})\right) \geqq m(\bar{F})+m(\bar{R}) \geqq$ $2+m(\bar{R})$ as $m(\bar{J})=m(\bar{L}) \geqq 2$. Since $m(\bar{T})=1+m(\bar{R}), m\left(C_{G}(e) \cap C_{G}(\bar{L})\right)>$ $m(T)$. If $m(T)=m(P)$ then (2) holds, so assume $m(T)<m(P)$ and pick $w \in N_{P}(Q)-Q$ with $w^{2} \in T . \quad T=C_{P}(e)$ implies $|T| \geqq 4$, and if $|T|=4$, then $P$ has maximal class and $m(P)=m(T)$. Thus $|T| \geqq 8$, $|R| \geqq 4$, and $R \cap R^{w} \neq 1$. As $R \leqq Q, R \cap R^{w} \supseteqq\langle Q, w\rangle$ and we can pick $z \in \operatorname{Inv}(R)$ with $\langle Q, w\rangle \subseteq C_{S}(z)$. By choice of $e, J$ corresponds isomorphically to a 2-component $V$ of $C_{G}(z)$. Let $J_{0}=J_{\langle b, z\rangle}$. It is easy to see that $\left\langle J_{0}^{L\left(C_{G}(z)\right)}\right\rangle=V$ and $\left\langle J_{0}^{L\left(C_{G}(b)\right)}\right\rangle=L L^{a}$. Likewise the normal closure of $J_{0}$ in $\left(L L^{a}\right)_{z}$ is $\left(L L^{a}\right)_{z}$ whence $\left(L L^{a}\right)_{z} \leqq L\left(C_{G}(z)\right)$ implies $\left(L L^{a}\right)_{z} \subseteq\left\langle J_{0}^{L\left(G_{G}^{(z) \prime}\right\rangle}\right\rangle=V$. But $\left|L L^{a}: O\left(L L^{a}\right)\right|>|J: O(J)|=|V: O(V)|$ and we see that $|T| \geqq 8$ forces $T=P$ and establishes (2).

LEMMA 3.5. Suppose $J$ is a 2-component of $C_{G}(a), a \in \operatorname{Inv}(G)$, and $S \in \operatorname{Syl}_{2}\left(C_{G}(a) \cap N_{G}(J)\right)$. Let $P=C_{S}(J / O(J))$. Assume that for every $b \in \operatorname{Inv}(P) J$ corresponds isomorphically to a 2-component $L$ of $C_{G}(b)$ and that if $b \in \operatorname{Inv}\left(C_{P}(S)\right)$, then $S \in \operatorname{Syl}_{2}\left(C_{G}(b) \cap N_{G}(L)\right)$. Under these conditions $J$ is maximal in $G$.

Proof. We must show that if $J \rightarrow K$, then $J / Z^{*}(J) \cong K / Z *(K)$. We are given a sequence of 2-components $J=L_{1}, \cdots, J_{r}=K$ such that for each pair $L_{i}, L_{i+1}$ these are involutions $a_{\imath}, b_{i+1}$ satisfying

(1) $L_{i}$ is a 2-component of $C_{G}\left(a_{i}\right)$;

(2) $L_{i+1}$ is a 2-component of $C_{G}\left(b_{i+1}\right)$;

(3) $\left[a_{i}, b_{i+1}\right]=1$;

(4) $L_{i} \rightarrow L_{i+1}$.

We will show that $L_{2}$ corresponds isomorphically to $L_{i+1}, 1 \leqq i \leqq$ $r-1$, which will suffice for the proof of the lemma. Assume $r$ is minimum such that for assertion fails. For any $i$ with $2 \leqq i \leqq r-1$ and $x \in N_{G}\left(L_{i}\right)$ we may replace $a_{j}$ by $a_{j}^{x}, i \leqq j \leqq r-1$, and $b_{j}, L_{j}$ by $b_{\jmath}^{x}, L_{j}^{x}$, respectively $i+1 \leqq j \leqq r$ to obtain another sequence of length 
$r$ for which our assertion fails. By (3) we can choose $x$ such that $\left\langle a_{j-1}, b_{j}, a_{j}^{x}\right\rangle$ is a 2-group. Thus we may assume $\left\langle a_{i-1}, b_{i}, a_{i}\right\rangle$ is a 2group for all $i$ with $2 \leqq i \leqq r-1$.

Pick $x_{i} \in \operatorname{Inv}\left(Z\left(\left\langle a_{i-1}, b_{i}, a_{i}\right\rangle\right)\right)$ and let $L_{i-1}$ correspond to the 2component $K_{i}$ of $C_{G}\left(z_{i}\right)$. As $i<r$, each term in the sequence $L_{1}, \cdots$, $L_{i-1}, K_{i}$ corresponds isomorphically to the next term. A straightforward argument using $L$-Balance shows that $K_{i}$ corresponds to $L_{i}$ and $L_{i+1}$. Thus by replacing $b_{i}, L_{i}$ by $z_{i}, K_{i}$ respectively we may assume $b_{i} \in Z\left(\left\langle a_{i-1}, b_{i}, a_{i}\right\rangle\right)$.

We wish to apply Lemma 3.3. If $b \in \operatorname{Inv}\left(C_{P}(S)\right)$ and $J$ corresponds to the 2-component $L$ of $C_{G}(b)$, then by hypothesis $S \in \operatorname{Syl}_{2}\left(C_{G}(b) \cap N_{G}(L)\right)$. Thus $S$ normalizes $J_{b} O\left(C_{G}(b)\right)=L O\left(C_{G}(b)\right)$. As $L=L\left(J_{b} O\left(C_{G}(b)\right)\right)$ is characteristic in $J_{b} O\left(C_{G}(b)\right)$, it follows that condition $\left.{ }^{* *}\right)$ of Lemma 3.3 holds.

Pick $T \in \operatorname{Syl}_{2}\left(N_{G}(J)\right)$ with $S \subseteq T . \quad S=C_{T}(a)$ implies $Z(T) \subseteq Z(S)$, so for $z \in \operatorname{Inv}(Z(T))$ our hypothesis implies $S \in \operatorname{Syl}_{2}\left(C_{G}(z) \cap N_{G}(L)\right)$ where $J$ corresponds isomorphically to the 2-component $L$ of $C_{G}(z)$. But $T$ normalizes $J_{z}$ whence $T$ normalizes $L$, and it follows that $S=T$. In particular $\left\langle a_{1}, b_{2}\right\rangle^{x} \subseteq P$ for some $x \in G$ and replacing our sequence of 2-components and involutions by their $x$-conjugates we may assume $\left\langle a_{1}, b_{2}\right\rangle \subseteq P$. Clearly we may further assume $a_{1}=a$. As $b_{2} \in P$, our hypotheses force $r \geqq 3$.

Apply Lemma 3.3 to $\left\langle b_{2}\right\rangle$. These exists $x \in G$ such that for $e=$ $\left(b_{2}\right)^{x}$ we have

(1) $e \in P$;

(2) $C_{S}(e) \subseteq \operatorname{Syl}_{2}\left(C_{G}(e) \cap N_{G}\left(J_{e} O\left(C_{G}(e)\right)\right)\right)$;

(3) $J_{e} O\left(C_{G}(e)\right)=\left(J_{b_{2}} O\left(C_{G}\left(b_{2}\right)\right)\right)^{x}$.

By (3) $L\left(J_{e} O\left(C_{G}(e)\right)\right)=\left(L_{2}\right)^{x}$, a 2-component of $C_{G}(e)$. Replacing $b_{j}$ by $b_{\jmath}^{x}, L_{j}$ by $\left(L_{j}\right)^{x}, 2 \leqq x \leqq r$ and replacing $a_{j}$ by $a_{j}^{x}, 2 \leqq j \leqq r-1$, we may deduce from (2) that

$$
Q=C_{S}\left(b_{2}\right) \in \operatorname{Syl}_{2}\left(C_{G}\left(b_{2}\right) \cap N_{G}\left(L_{2}\right)\right) .
$$

As $r \geqq 3$, we have $b_{2} \in Z\left(\left\langle a, b_{2}, a_{2}\right\rangle\right)$. Whence $\left(a_{2}\right)^{y} \in Q$ for some $y \in$ $C_{G}\left(b_{2}\right) \cap N_{G}\left(L_{2}\right)$. As before we may assume $a_{2} \in Q . \quad\left[a, a_{2}\right]=1$ implies that we may replace $b_{2}$ by $a_{2}$ and assume $b_{2}=a_{2}$. Since $b_{3}$ normalizes $L_{2}$ and centralizes $a_{2}, b_{3}^{w} \in Q$ for some $w \in C_{G}\left(a_{2}\right) \cap N_{G}\left(L_{2}\right)$. Again we may assume $b_{3} \in Q$. Now by hypotheses $J$ corresponds isomorphically to $L_{3}$. Thus $r \geqq 4$ and the sequence $J=L_{1}, L_{3}, \cdots, L_{r}=K$ contradicts our choice of $r$.

LEMмA 3.6. Let $G$ be a group in which all 2-local subgroups satisfy the $B(G)$-Conjecture. Suppose $J$ is a 2-component of $C_{G}(a)$, $a \in \operatorname{Inv}(G)$, and $S \in \operatorname{Syl}_{2}\left(C_{G}(a) \cap N_{G}(J)\right)$. Let $P=C_{S}(J / O(J))$. Assume the following conditions: 
(i ) If $b \in \operatorname{Inv}\left(C_{P}(S)\right)$, then $S \in \operatorname{Syl}_{2}\left(C_{G}(b)\right) \cap N_{G}\left(J_{b} O\left(C_{G}(b)\right)\right)$.

(ii) For some $b \in \operatorname{Inv}(P), J$ does not correspond isomorphically to any 2-component of $C_{G}(b)$.

(iii) $m(J) \geqq 2$.

Under these conditions these exists a 2-group $B, 1 \neq B \subseteq P$ for which the following conclusions hold where $H=N_{G}(B), \bar{H}=H / O_{2^{\prime}, 2}(H), V=$ $\left\langle J^{L(H)}\right\rangle$.

(1) $\overline{J_{B}}$ is a component of $C_{\bar{H}}(\bar{a})$ and is standard in $\bar{H}$;

(2) $V=[V, a]=L(H)$;

(3) $B \in \operatorname{Syl}_{2}\left(C_{H}(V / O(V))\right)$ and $N_{S}(B) \in \operatorname{Syl}_{2}\left(N_{G}(B) \cap\right.$ $\left.N_{G}\left(J_{B} O\left(C_{G}(B)\right)\right)\right)$;

(4) If $E \subseteq P$ with $|E|>|B|$ or with $|E|=|B|$ and $\left|N_{S}(E)\right|>$ $\left|N_{S}(B)\right|$, then $J$ corresponds isomorphically to a 2-component of $N_{G}(E)$.

Proof. Pick $B_{1} \subseteq P$ maximal in the partial order indicated in (4) such that $H_{1}=N_{G}\left(B_{1}\right)$ and $V_{1}=\left\langle J_{B_{1}}^{L\left(H_{1}\right)}\right\rangle$ satisfy $V_{1}=\left[V_{1}, a\right]$. By (ii) and $L$-Balance we have that $B_{1} \neq 1$ and (4) holds. By Lemma 3.3 we can find $x \in G$ such that for $B_{1}^{x}=B$ we have

(a) $N_{S}(B) \in \operatorname{Syl}_{2}\left(N_{G}(B) \cap N_{G}\left(J_{B} O\left(C_{G}(B)\right)\right)\right)$.

(b) $N_{S}\left(B_{1}\right)^{x} \cong S$.

(c) $J_{B} O\left(C_{G}(B)\right)=\left[J_{B_{1}} O\left(C_{G}\left(B_{1}\right)\right)\right]^{x}$.

Let $H=N_{G}(B)$ and $V=\left\langle J_{B}^{L(H)}\right\rangle . \quad V_{1}^{x}$ is the normal closure of $\left(J_{B_{1}}\right)^{x}$ in $L(H)$. By (c) $V_{1}^{x} \subseteq\left\langle\left(J_{B} O\left(C_{G}(B)\right)\right)^{L(H)}\right\rangle$. As $V_{1}^{x}$ is a product of 2components of $L(H)$, it follows that $V_{1}^{x} \subseteq V$. In particular $\left|V_{1}: Z^{*}\left(V_{1}\right)\right|>$ $\left|J: Z^{*}(J)\right|$ implies the same for $V$ whence $V=[V, a]$. Let $Q=N_{S}(B)$. By (a) $Q \cap C_{H}(V / O(V)) \in \operatorname{Syl}_{2}\left(C_{H}(V) O(V)\right)$, and maximality of $|B|=$ $\left|B_{1}\right|$ implies $B=C_{Q}(V / O(V))$. Now (2) and (3) are immediate and (b) implies (4). Also by (3) $B \in \operatorname{Syl}_{2}\left(O_{2^{\prime 2}}(H)\right)$.

$J_{B}$ is a 2-component of $C_{G}(B) \cap C_{G}(a)=C_{G}(B\langle a\rangle)$. Consequently $J_{B}$ is a 2-component of $N_{G}(B\langle a\rangle)$. From the structure of $H, \overline{N_{H}(B\langle a\rangle)}=$ $N_{\bar{H}}(\overline{B\langle a\rangle})=C_{\bar{H}}(\bar{a})$. Thus $\overline{J_{B}}$ is a 2-component of $C_{\bar{H}}(\bar{a}) . \quad$ As $H$ satisfies the $B(G)$-conjecture, $\left[J_{B}, O\left(J_{B}\right)\right] \cong O(H)$ whence $\overline{J_{B}}$ is quasisimple and $\overline{J_{B}}$ is a component of $C_{\bar{H}}(\bar{a})$. We claim that if $\bar{t} \in \operatorname{Inv}\left(C_{\bar{H}}\left(\overline{J_{B}}\right)\right)$, then $\overline{J_{B}}$ is a component of $C_{\bar{H}}(\bar{t})$. Let $Y=J_{B} O_{2^{\prime}, 2}(H)$. As $J_{B} O(H)=O(Y) L(Y)$ is characteristic in $Y$, (3) implies $Q \in \operatorname{Syl}_{2}\left(N_{H}(Y)\right)$. Accordingly $\bar{Q} \in$ $\operatorname{Syl}_{2}\left(N_{\bar{H}}\left(\bar{J}_{B}\right)\right)$ and we may assume $t \in C_{Q}\left(\overline{J_{B}}\right)$ projects onto $\bar{t}$. We have $t^{2} \in Q \cap O_{2^{\prime}, 2}(H)=B$. Let $T=\langle t, B\rangle$; by maximality of $|B|, N_{G}(T)$ has a 2-component $L$ with $J_{T} \subseteq L, J_{T} O(L)=L$. Repeating the argument we used for $B\langle a\rangle$, we see that $\bar{L}$ is a component of $C_{\bar{H}}(\bar{t})$. Since $\bar{L}$ is quasisimple, $\bar{L}=\overline{J_{T}} O(\bar{L})$ implies $\bar{L}=\overline{J_{T}}$. Likewise $\overline{J_{B}}=\overline{J_{T}}=\bar{L}$ and our claim is proved.

Now by [8, Proposition 4.1] Theorem 5 of [2] applies to a maximal product of pairwise commuting $\bar{H}$-conjugates of $\bar{J}$. From (2) and 
hypothesis (iii) we have $\left[\bar{J}, \bar{J}^{\bar{h}}\right] \neq 1$ for any $\bar{h} \in \bar{H}$. Further if $\bar{t} \in$ $C_{\bar{H}}(\bar{J}) \cap C_{\bar{H}}\left(\bar{J}^{\bar{h}}\right)$, then $\bar{J}$ and $\bar{J}^{\bar{h}}$ are components of $C_{\overline{I I}}(\bar{t})$, which forces $\bar{J}=\bar{J}^{\bar{h}}$ (else $\left[\bar{J}, \bar{J}^{\bar{h}}\right]=1$ ) whence (1) holds.

LEMMA 3.7. Let $G$ be a group with commuting involutions a and $x$, and let $D=O\left(C_{G}(x)\right) \cap C_{G}(a)$. Either $D \subseteq O\left(C_{G}(a)\right)$ or there is a 2component $J$ of $C_{G}(a)$ such that $[J, D]=J=[J, x]$.

Proof. See [28, Lemma 2.5].

LEMMA 3.8. Let $(a, x, J)$ be an unbalancing triple of $G$. If $b \in$ Inv $\left(C_{G}(a)\right)$ with $[b, x]=1,[b, J] \subseteq O(J)$ and $J$ corresponds to the 2component $L$ of $C_{G}(b)$, then $(b, x, L)$ is an unbalancing triple of $G$.

Proof. Let $F=O\left(C_{G}(x)\right) \cap C_{G}(a)$. $\quad F$ acts nontrivially on $J / O(J)$ and as $[J, b] \leqq O(J),[F, b]$ centralizes $J / O(J) . \quad[x, b]=1$ implies that $b$ acts on $F$, so $F=C_{F}(b)[F, b]$ and $E=C_{F}(b)$ acts nontrivially on $J / O(J)$. Hence $E$ acts nontrivially on $J_{b} / O\left(J_{b}\right)$ and $L L^{a} / O\left(L L^{a}\right)$. Note that $L L^{a}=\left\langle J_{b}^{\left.L\left(C_{G}(b)\right)\right\rangle}\right.$ implies that $E$ normalizes $L L^{a}$. Likewise $\langle x\rangle$ acts nontrivially on $L L^{a} / O\left(L L^{a}\right)$. Now apply Lemma 3.7 to the group $L L^{a} E\langle a, x, b\rangle$ to obtain the desired conclusion.

Lemma 3.9. Let $G$ be a group such that all 2-local subgroups satisfy the $B(G)$-Conjecture and such that for every unbalancing triple $(a, x, J), m(J) \geqq 2$. For any unbalancing triple $(a, x, J)$ there exists a maximal unbalancing triple $(b, y, L)$ with $J \rightarrow L$.

Proof. It suffices to show that if $(a, x, J)$ is not maximal, then there is an unbalancing triple $(b, y, L)$ with $J \rightarrow L$ such that one of the following occurs:

(1) $|L: O(L)|>|J: O(J)|$;

(2) $|L: O(L)| \geqq|J: O(J)|$ and $m\left(C_{G}(b) \cap C_{G}(L / O(L))\right)>$ $m\left(C_{G}(a) \cap C_{G}(J(O(J)))\right):$

(3) $|L: O(L)| \geqq|J: O(J)|$ and $m\left(C_{G}(b) \cap C_{G}(L / O(L))\right) \geqq$ $m\left(C_{G}(a) \cap C_{G}(J(O(J)))\right)$ and $\left|C_{G}(b) \cap N_{G}(L)\right|_{2}>\left|C_{G}(a) \cap N_{G}(J)\right|_{2}$.

Pick $b \in \operatorname{Inv}\left(C_{G}(a) \cap C_{G}(J / O(J))\right)$ so that condition (1) or (2) of the definition of maximal unbalancing triple fails. Pick $S \in \operatorname{Syl}_{2}\left(C_{G}(a) \cap\right.$ $\left.N_{G}(J)\right)$ with $Q=C_{S}(b) \in \operatorname{Syl}_{2}\left(C_{G}(\langle a, b\rangle) \cap N_{G}(J)\right)$. Let $P=C_{S}(J / O(J))$.

If condition (1) fails, $G$ has an unbalancing triple $(b, y, L)$ such that $J$ corresponds to $L$ and $y \in C_{G}(a) \cap N_{G}(J)$. Thus $y \in C_{G}(\langle a, b\rangle) \cap$ $N_{G}(J)$ and we may assume $y \in Q$. If condition (2) fails, then $b \in Z(S)$, and as we may assume by conjugation in $C_{G}(a) \cap N_{G}(J)$ that $x \in S$, Lemma 3.8 guarantees that $(b, x, L)$ is an unbalancing triple where $L$ is any 2-component of $C_{G}(b)$ to which $J$ corresponds. In either case 
if $J$ does not correspond isomorphically to $L$, apply Lemma 3.4 to find $b_{1} \in \operatorname{Inv}(P)$ with $Q \subseteq C_{S}\left(b_{1}\right)$ such that $J$ corresponds to the 2-component $L_{1}$ of $C_{G}\left(b_{1}\right)$ and either (1) or (2) above holds with $b_{1}$ and $L_{1}$ in place of $b$ and $L$ respectively. By Lemma 3.8 again either $\left(b_{1}, y, L_{1}\right)$ or $\left(b_{1}, x, L_{1}\right)$ is an unbalancing triple.

Thus we may assume that $J$ corresponds isomorphically to $L$. Thus it is condition (2) which fails; $b \in Z(S)$ but $S \notin \operatorname{Syl}_{2}\left(C_{G}(b) \cap N_{G}(L)\right.$ ). But again $(b, x, L)$ is an unbalancing triple, and as $J$ corresponds isomorphically to $L$, it is clear that (3) above holds.

Finally, the following result from [25] will be used. For any group $G$ let $G^{2}=\left\langle g^{2} \mid g \in G\right\rangle$.

Lemma 3.10. Let $G$ be a group with $P \in \operatorname{Syl}_{2}(G)$ and $Q \subseteq P$ weakly closed in $P$ with respect to $G$. Suppose $x \in \operatorname{Inv}\left(Q \cap G^{2}\right)-N_{G}(Q)^{2}$. Then there exists a group $S \subseteq Q$ such that

(1) $C_{Q}(x) \cong S$.

(2) The transfer $V_{P \rightarrow S}(x) \notin \Phi(Q)$.

4. Proof of Theorem A. As we noted after Lemma 1.1 it suffices to prove the Unbalanced Group Conjecture for a minimal unbalanced group $G$. Let $(a, x, J)$ be an unbalancing triple in $G$. By Lemma 3.1 we may assume $F^{*}(G)$ is simple whence $Y=$ $\left\langle x, O\left(C_{G}(x)\right) \cap C_{G}(a), J\right\rangle$ is a proper unbalanced subgroup of $G$ as $Y \subseteq$ $C_{G}(a) \subset G$. Applying the U.G.-Conjecture to $Y$, we see that $J / Z^{*}(J)$ is isomorphic to one of the simple groups listed in the conclusion of the U.G.-Conjecture: By Theorems 1.2-1.5 $J / O(J)$ is isomorphic to $L_{2}(q), q$ odd, $A_{7}, L_{3}(4)$ or $H e$.

By Lemma 3.9 we may assume that $(a, x, J)$ is a maximal unbalancing triple in G. Assume Hypothesis S holds. By Theorem B, either we have the hypotheses of Theorem 1.7 satisfied by $G$ or we have the hypotheses of Lemma 3.5 satisfied by $J$ and $G$. In any case, by applying Theorem 1.7 we may assume that

(1) Either $J / Z^{*}(J) \cong L_{3}(4)$ or $J / O(J) \cong H e$.

(2) $J$ is maximal in $G$.

(3) If $(b, y, K)$ is any unbalancing triple in $G$, then $K / Z^{*}(K)$ is isomorphic to $L_{2}(5), L_{2}(7), L_{3}(4)$ or $\mathrm{He}$.

Now $G$ satisfies the hypotheses of Theorem 1.6. Thus $F^{*}(G)$ is isomorphic either to $L_{3}(4)$ or to $\mathrm{He}$. Thus the Unbalanced Group Theorem is proved.

5. The proof of Theorem B, Part 1. Throughout this section and the next, $G$ will be a fixed counterexample to Theorem B and $(a, x, J)$ will be a fixed maximal unbalancing triple of $G$ with $J / O(J)$ isomorphic to $A_{7}$ or to $L_{2}(q), q$ odd, or $J / Z^{*}(J)$ isomorphic to $L_{3}(4)$. 
Pick $S \in \operatorname{Syl}_{2}\left(C_{G}(a) \cap N_{G}(J)\right)$ with $x \in S$ and define $D=S \cap J, P=$ $C_{S}(J / O(J))$. Conclusion (3)(b) of Theorem B is part of the definition of maximal unbalancing triple, so we assume that $(a, x, J)$ does not satisfy conclusion $(3)(a)$. Choose $B \cong P$ to satisfy the conclusion of Lemma 3.6, and let $V=\left\langle J_{B}^{L\left(N_{G}(B)\right)}\right\rangle$. From conclusion (1) of Lemma 3.6 and Hypothesis (c) of Theorem B we have that $V / O_{2^{\prime}, 2}(V)$ and hence $V$ itself are products of groups of restricted type. In fact by $L$-Balance, $V=K K^{a}$ where $K$ is a 2-component of $N_{G}(B)$ and if $K \neq K^{a}$, then $K / Z^{*}(K) \cong J / Z^{*}(J)$. We prove Theorem B by considering the possibilities for $K$ and showing that each possibility leads to a contradiction. By Lemma 3.1 we may suppose that $F^{*}(G)$ is simple.

\section{Lemma 5.1. $B \cap B^{x}=1$.}

Proof. If not, then as $x^{2}=1$, we may choose $b \in \operatorname{Inv}\left(C_{B}(x)\right)$. By Lemma 3.8 and the definition of maximal unbalancing triple, $J$ corresponds isomorphically to a 2-component $L$ of $C_{G}(b)$. In particular $[L, a] \subseteq O(L)$. Now

$$
J_{B}=O^{2^{\prime}}\left(C_{J}(B)\right) \subseteq O^{2^{\prime}}\left(C_{J}(b)\right)=J_{b} \subseteq L .
$$

As $L\left(N_{G}(B)\right)=L\left(C_{G}(B)\right)$ and $L\left(C_{G}(B)\right) \subseteq L\left(C_{G}(b)\right)$ by $L$-Balance, we have

$$
K=\left\langle J_{B}^{L\left(G_{G^{(B)}}\right)}\right\rangle \leqq\left\langle J_{b}^{L\left(G_{G}^{(b))}\right\rangle=L .}\right.
$$

From Lemma 3.6, $K K^{a}=\left[K K^{a}, a\right]$, whence $K K^{a} \subseteq[L, a] \subseteq O(L)$, which is impossible. We conclude that $B \cap B^{x}=1$.

We now know that $G$ satisfies the following hypothesis with $G$ in place of $H$.

Hypothesis 5.2. $H$ is a group such that

(1) All proper sections of $H$ satisfy the U.G.-Conjecture;

(2) The solution to the standard component problem for $L_{2}(q)$, $q$ odd, $A_{7}$ and $L_{3}(4)$ in proper sections of $H$ is a central product of groups of restricted type;

(3) There exists $a \in \operatorname{Inv}(H)$ and $J$ a 2-component of $C_{H}(a)$ with $J / O(J) \cong L_{2}(q), q$ odd, $J / O(J) \cong A_{7}$, or $J / Z^{*}(J) \cong L_{3}(4)$;

(4) For any $S \in \operatorname{Syl}_{2}\left(C_{H}(a) \cap N_{H}(J)\right)$ with $P=C_{S}(J / O(J))$, there exists $B \subseteq P$ with $B \neq 1$ such that for $W=N_{H}(B)$ and $\bar{W}=W / O_{2^{\prime}, 2}(W)$, $\bar{J}_{B}$ is a component of $C_{\bar{W}}(\bar{a})$ and is standard in $\bar{W}$; and further

(5) For $K K^{a}=\left\langle J_{B}^{L(W)}\right\rangle$, we have $K K^{a}=\left[K K^{a}, a\right]=L(W)$;

(6) $B \in \operatorname{Syl}_{2}\left(C_{W}\left(K K^{a} / O\left(K K^{a}\right)\right)\right)$;

(7) If $E \cong P$ with either $|E|>|B|$ or $|E|=|B|$ and $\left|N_{S}(E)\right|>$ $\left|N_{S}(B)\right|$, then $J$ corresponds isomorphically to a 2-component of $N_{G}(E)$;

(8) There exists $x \in S$ with $B \cap B^{x}=1$ and $x^{2} \in N_{P}(B)$; 
(9) $N_{S}(B)$ contains a Sylow 2-subgroup of $L(W) \cap N_{H}\left(J_{B} O(W)\right)$.

By the hypothesis of Theorem B, G satisfies conditions (1)-(3) of Hypothesis 5.2. Hypothesis (a) of Theorem B, Lemma 3.6 and the assumption that $G$ fails conclusion (1) of Theorem B give (4)-(7) and (9). Lemma 5.1 implies (8).

The advantage of Hypothesis 5.2 is that it is inherited by certain sections of $H$. Thus we may argue in certain sections of $G$ which do not have an unbalancing triple.

Lemma 5.3. Let $H$ satisfy Hypothesis 5.2 and let $a^{g} \in S-P$ for some $g \in N_{H}(B)$. The following conditions hold:

(1) $B=C_{P}\left(a^{g}\right) \cap C_{P}\left(J^{g} / O\left(J^{g}\right)\right)$;

(2) $a^{g} \notin C_{\measuredangle D, a}(x)$.

Proof. Let $a^{g}=e$. Since $g \in N_{H}(B)$, clearly $B \subseteq C_{P}(e) \cap C_{P}\left(J^{g} / O\left(J^{g}\right)\right)$. Suppose that

$$
B \subset E \subseteq C_{P}(e) \cap C_{P}\left(J^{g} / O\left(J^{g}\right)\right)
$$

with $|E: B|=2$. By 5.2(7) $J$ corresponds isomorphically to a 2-component $L$ of $C_{H}(E)$. As $[e, E]=1=[e, a], e$ normalizes $J_{E}$ and so $L^{e}=L$. Likewise $e \in S-P$ implies $L=[L, e]$. Let $M=J^{g}$ correspond to the 2-component $N$ of $N_{G}(E)$. Our conditions imply $N \neq L \neq N^{e}$, else $N=N^{e}=L$ and $M / O(M) \cong J / O(J) \cong L / O(L)$ would force $e$ to centralize $L / O(L)$. Thus $\left[M_{E}, J_{E}\right] \subseteq O(N)$, and defining $Y=\left\langle M_{E}, J_{E}\right\rangle$, we have

$$
Y / O(Y)=M_{E} O(Y) / O(Y) \times J_{E} O(Y) / O(Y) .
$$

Further, $O(Y) \subseteq O\left(L\left(N_{H}(E)\right)\right)=O\left(L\left(C_{H}(E)\right)\right)$.

Let $V=N_{H}(B)$ and $\bar{V}=V / O(V)$. Since $|E: B|=2, E \subseteq V$. Since $C_{\bar{V}}(\bar{E})=\overline{C_{V}(E)}=\overline{C_{H}(E)}, \overline{L\left(C_{H}(E)\right)}=L\left(C_{\bar{V}}(\bar{E})\right)$. By $5.2(1) \quad \bar{V}$ satisfies the $B(G)$-Conjecture, whence

$$
\left[\overline{L\left(C_{H}(E)\right)}, O\left(\overline{\left.L\left(C_{H}(E)\right)\right)}\right]=1 .\right.
$$

Consequently $[\bar{Y}, O(\bar{Y})]=1$ and $\bar{Y}$ is the central product of $\bar{J}_{E}$ and $\bar{M}_{E}$. By Lemma 3.2, $\bar{J}_{E}=\bar{J}_{B}$ and $\bar{M}_{E}=\bar{M}_{B}=\bar{M}_{B^{g}}=\left(\bar{J}_{B}\right)^{g}$. Thus $\left[\bar{J}_{B}, \bar{J}_{B}^{\bar{g}}\right]=1$ contradicting $5.2(4)$. This proves that there is no such $E$ and (1) holds.

Suppose $e \in C_{\langle D, a\rangle}(x)$. If $x$ normalizes $M=J^{g}$, then $x$ normalizes $B$ by (1). But $B \cap B^{x}=1$ by $5.2(8)$, so $M^{x}$ is a 2-component of $C_{H}(e)$ distinct from $M$. Thus $L\left(C_{H}(a)\right)$ is a product of 2-components $J L_{1} \cdots L_{t}$ with $t \geqq 1$ and we may take $L_{1}$ to be a $C_{H}(a)$-conjugate of $J$. Further $e$ centralizes $L_{i} / O\left(L_{i}\right), 1 \leqq i \leqq t$. Let $P_{i}=P \cap L_{i} \in \operatorname{Syl}_{2}\left(L_{i}\right)$.

Suppose $\left(L_{i}\right)^{x}=L_{j}$; then $x$ normalizes $P_{i} P_{j}$, so $P_{i} J_{j} \nsubseteq B$. As $e \in$ 
$\langle D, a\rangle,\left[P_{i} P_{j}, e\right]=1$, so by (1) $P_{i} P_{j}$ must act nontrivially on $M / O(M)$. By $L$-Balance, $\left(L_{i}\right)_{e}$ or $\left(L_{j}\right)_{e}$ is a 2-component of $L\left(C_{M M^{a}}(a)\right)$. As $M / O(M)$ is known by $5.2(3), L\left(C_{M M^{a}}(a)\right)$ has at most one component, and it follows that $t=1$ or $t=2$ and $L_{2}=\left(L_{1}\right)^{x}$.

In either case, the 2-components of $C_{H}(a)$ are all conjugate in $C_{H}(a)$ and hence isomorphic. We may assume that $P_{1}$ acts nontrivially on $M / O(M)$. By $L$-Balance, $\left(L_{1}\right)_{e} \subseteq M M^{a}$. If $M=M^{a}$, then $L_{1}$ corresponds isomorphically to $M$, whence $a \in B$ by (1). But this is not the case. If $M \neq M^{a}$, let $y=a^{g^{-1}}$. Clearly $[y, a]=[a, e]^{g^{-1}}=1$ and $\left[J, J^{y}\right] \subseteq O\left(L\left(C_{G}(a)\right)\right)$. But now since $y \in N_{H}(B)$, we may argue as in the proof of (1) with $\langle B, y\rangle$ in the role of $E$ and reach a contradiction to $5.2(4)$.

Lemma 5.3(2) puts a severe restriction on the fusion of $a$ in $N_{G}(B)$. Using this fact and the properties of $K$ listed in Proposition 2.3, we can immediately rule out many possibilities for $K$.

Lemma 5.4. Let $H$ satisfy Hypothesis 5.2. The possibilities for $J / O(J)$ and $K K^{a} / O\left(K K^{a}\right)$ are as follows:
(1) $\frac{J / O(J)}{A_{n}, n=5,6,7}$
$\underline{K K^{a} / O\left(K K^{a}\right)}$
(2) $L_{2}(5), L_{2}(7)$
(1) $A_{n+2}, A_{n+4}, \hat{A}_{n} * \hat{A}_{n}$
(3) $L_{3}^{\wedge}(4)$
(2) A proper covering group of $L_{3}(4)$
(4) $L_{2}\left(q^{2}\right), q$ odd
(3) A proper covering group of Suz
(5) $L_{2}(q), q$ odd,$q>3$
(4) A proper covering group of $L_{4}(q)$, $U_{4}(q)$ or $\Omega^{-}\left(8, q^{1 / 2}\right)$
(5) $L_{2}\left(q^{2}\right)$ with no diagonal automor- phism
(6) $L_{2}(q), q$ odd $q>3$
( 7 ) $L_{2}\left(q^{2}\right), q$ odd .
(6) a central product $\mathrm{SL}(2, q) * \mathrm{SL}(2, q)$
( 7 ) $\operatorname{PSp}(4, q)$.

In the remainder of this section we shall develop some more general lemmas and use these to eliminate cases (1)-(4) on the above list with the exception of the case $J / O(J) \cong A_{6}, K / O(K) \cong A_{8}$. Cases (5)-(7) cause the greatest difficulties and are deferred to the next sections.

We fix the notation $Q=N_{S}(B), T=Q \cap P$.

Lemma 5.5. Let $H$ satisfy Hypothesis 5.2. Then $K K^{a} / O\left(K K^{a}\right) \not$ Ŝैz and $Z(J / O(J))=1$.

Proof. By Lemma 5.4, the first assertion implies the second. Suppose $K K^{a} / O\left(K K^{a}\right) \cong S \hat{u} z$. Then $K=K^{a}$ and by Lemma $5.4,\left|Z^{*}(K)\right|$ is even. By Proposition 2.4(c), $Z^{*}\left(L\left(C_{K}(a)\right)\right)$ has even order and lies in $Z^{*}(K)$. From $L$-balance, $Z^{*}(J)$ has even order. As $B$ centralizes 
$J / O(J), Q \cap J \in \operatorname{Syl}_{2}(J)$ and $Q \cap Z^{*}(J) \in \operatorname{Syl}_{2}\left(Z^{*}(J)\right)$. Thus $Q \cap Z^{*}(J)=$ $S \cap Z^{*}(J)$ is normalized by $x$. But $Q \cap Z^{*}(J) \cong Z^{*}\left(J_{B}\right) \cong Z^{*}(K)$, whence $Q \cap Z^{*}(J) \subseteq B$ by 5.2(6), contradicting 5.2(8).

Lemma 5.6. Let $H$ satisfy Hypothesis 5.2. The following conditions hold:;

(1) $C_{P}(S)=\langle a\rangle$

(2) $J \leqq C_{G}(a)$ and $S \in \operatorname{Syl}_{2}\left(C_{G}(a)\right)$;

(3) If $L$ is a 2-component of $C_{G}(a)$ distinct from $J$, then $Z(L / O(L)) \neq 1$.

Proof. From 5.2(9) we see that $Q$ contains a Sylow 2-subgroup of $L(W) \cap N_{H}\left(J_{B} O\left(N_{H}(B)\right)\right)$. As $J_{B} O\left(N_{H}(B)\right)$ is characteristic in $J_{B} O_{2^{\prime}, 2}\left(N_{H}(B)\right), Q \cap L(W)$ projects onto a Sylow 2-subgroup of $N_{\overline{L(W)}}\left(\bar{J}_{B}\right)$ in the notation of 5.2(4). From 5.2(6) we have

$$
B=C_{Q}\left(K K^{a} / O\left(K K^{a}\right)\right)=C_{Q}\left(K K^{a} / O_{2^{\prime}, 2}\left(K K^{a}\right)\right) .
$$

It follows now from Proposition 2.3(2) that

$$
C_{P}(S) \subseteq C_{T}(Q) \subseteq\langle a, B\rangle \text {. }
$$

Now (1) follows from Lemma 5.1. If $L$ is as in (3), then $\Omega_{1}\left(C_{P}(S)\right) \cap$ $\left\langle L^{S}\right\rangle \neq\langle 1\rangle$ implies that $a \in\left\langle L^{S}\right\rangle$, whence $Z\left(\left\langle L^{S}\right\rangle / O\left(\left\langle L^{S}\right\rangle\right)\right) \neq 1$ and (3) holds. Then (3) and Lemma 5.5 imply (2).

\section{LEMMA 5.7. Let $H$ satisfy Hypothesis 5.2.}

(1) If $g \in N_{H}(B)$ and $a^{g} \in S-P$, then $C_{S}\left(a^{g}\right) \subseteq Q$.

(2) If $g \in H$ and $a^{g} \in\langle J, a\rangle-\langle a\rangle$, then $P \cap P^{g}=1$.

Proof. Let $L=a^{g}$ and $E=C_{S}(e) . \quad$ By Lemma 5.6(2), $J^{g} \supseteqq C_{G}(e)$; so $E$ normalizes $J^{g}$. By Lemma 5.3(1), $E \subseteq N_{S}(B)=Q$. This proves (1).

Suppose $F=P \cap P^{g} \neq 1$ in (2). As $F \leqq P, J_{F}$ covers $J / O(J)$ and $J_{F}=O^{\prime}\left(C_{J}(F)\right)$. As $e \in C_{\langle J, a\rangle}(F), e \in\left\langle J_{F}, a\right\rangle$; likewise $D \in \operatorname{Syl}_{2}\left(J_{F}\right)$. By Lemmas 5.4 and 5.5, $J$ has one class of involutions. Hence by replacing $g$ by $g h$ for some $h \in J_{F}$ we may assume that $e \in\left\langle C_{D}(S), a\right\rangle-\langle a\rangle$.

By Lemma 5.6(2), $S \in \operatorname{Syl}_{2}\left(C_{G}(a)\right)$. Likewise as $S \cong C_{G}(e), \quad S \in$ $\operatorname{Syl}_{2}\left(C_{G}(e)\right)$ and $S$ acts on $J^{g}$. Thus $U=\left\langle F^{S}\right\rangle$ centralizes $J / O(J)$ and $J^{g} / O\left(J^{g}\right)$. Consequently $U \subseteq P \cap C_{S}\left(J^{g} / O\left(J^{g}\right)\right)$ and $U \leqq S$ implies $U \cap$ $Z(S) \neq 1$. From Lemma 5.6(1) applied to $C_{H}(a)$ and to $C_{H}(e)$ we obtain $\langle a\rangle=\Omega_{1}\left(C_{U}(S)\right)=\langle e\rangle$, a contradiction. We conclude that $P \cap P^{g}=1$.

At this point we know little about the structure of $P$. When $P \subseteq Q=N_{S}(B), B^{x}$ normalizes $B$ and as $B^{x} \cap B=1$, we have $B B^{x}=$ $B \times B^{x}$. Thus $B$ is isomorphic to a subgroup of $T / B$; in particular, 
if $|T: B|=2$, then $|T|=4$ and $P$ has maximal class. In the case where $P \nsubseteq Q$, we attempt to recover this advantage in a section of $G$. We define an element $x_{0} \in S$ as follows:

$$
\begin{aligned}
& x_{0}=x \quad \text { if } \quad B \leqq P \\
& x_{0} \in P-N_{P}(B) \text { otherwise } \\
& \text { with } x_{0}^{2} \in N_{P}(B) .
\end{aligned}
$$

We let $B_{0}=B \cap B^{x_{0}}, S_{0}=N_{S}\left(B_{0}\right), P_{0}=S_{0} \cap P, J_{0}=J_{B_{0}}, G_{0}=N_{G}\left(B_{0}\right)$ and $\bar{G}_{0}=G_{0} / B_{0}$. Note $\left[D, B_{0}\right]=1$ implies $D=S_{0} \cap J_{B_{0}}$. $\bar{G}_{0}$ will be the appropriate section of $G$.

LEMMA 5.8. $\quad \bar{G}_{0}$ satisfies Hypothesis 5.2 with $H$ replaced by $\bar{G}_{0}$, $a$ by $\bar{a}, J$ by $\bar{J}_{0}, B$ by $\bar{B}, S$ by $\bar{S}_{0}, K$ by $\bar{K}_{0}$ and $x$ by $\bar{x}_{0}$.

Proof. We consider conditions (1)-(9) in turn; 5.2(1) and 5.2(2) are immediate. As $a \in B_{0}, \bar{a} \notin B_{0}, \bar{a} \in \operatorname{Inv}\left(\bar{G}_{0}\right) . \quad J_{0}$ is a 2-component of $C_{G}\left(\left\langle B_{0}, a\right\rangle\right)$ and hence of $N_{G}\left(\left\langle B_{0}, a\right\rangle\right)$. It follows that $\bar{J}_{0}$ is a 2-component of $\overline{N_{G}\left(\left\langle B_{0}, a\right\rangle\right) \cap G_{0}}=C_{\bar{G}_{0}}(\bar{a})$. Clearly $J / Z^{*}(J) \cong \bar{J}_{0} / Z^{*}\left(\bar{J}_{0}\right)$, so 5.2(3) holds. Since $K K^{a}=L\left(N_{G}(B)\right),\left[K K^{a}, B\right]=1$. Thus $K K^{a}=$ $L\left(N_{G_{0}}(B)\right)$. As $N_{\bar{G}_{0}}(\bar{B})=\overline{N_{G_{0}}(B)}$, it is straightforward to check 5.2(4)5.2(6) and 5.2(9). To check 5.2(7) suppose $\bar{E} \cong \bar{P}_{0}$ with $|\bar{E}|>|\bar{B}|$ or $|\bar{E}|=|\bar{B}|$ and $\left|N_{\bar{S}_{0}}(\bar{E})\right|>|\bar{Q}|$. Letting $E$ be the inverse image of $E$ in $P_{0}$, we can apply $5.2(7)$ to obtain that $J$ corresponds isomorphically to a 2-component $L$ of $N_{G}(E)$. As $\left[L, B_{0}\right] \leqq[L, E]=1, L$ is a 2-component of $N_{G_{0}}(E)$ whence $\bar{L}$ is a 2-component of $N_{\bar{G}_{0}}(\bar{E})$. As $\bar{J}_{E}=\bar{L}_{a}$ lies in $C_{\bar{c}_{0}}(\langle\bar{a}, \bar{E}\rangle)$ and covers $\bar{J}_{0} / O\left(\bar{J}_{0}\right)$ and $\bar{L} / O(\bar{L})$, it is clear from $L$-Balance that $\bar{J}_{0}$ corresponds isomorphically to $\bar{L}$. Finally 5.2(8) is immediate from our choice of $x_{0}$ and $B_{0}$ and Lemma 5.8 is proved.

It is easy to see that if $H$ satisfies Hypothesis 5.2, then so does $H / O(H)$. By Lemma 5.8, $\bar{G}_{0} / O\left(\bar{G}_{0}\right)$ satisfies Hypothesis 5.2 and, by our choice of $x_{0}$ and $B_{0}, \bar{G}_{0} / O\left(\bar{G}_{0}\right)$ satisfies the following hypothesis.

Hypothesis 5.9. $H$ is a group such that in the notation of Hypothesis 5.2

(1) $H$ satisfies Hypothesis 5.2;

(2) $O(H)=1$;

(3) Either $x \in N_{P}(Q)$ or $P \subseteq Q, x^{2}=1$ and $O\left(C_{J}(x)\right) \nsubseteq O(J)$.

Lemma 5.10. Let $H$ be a group satisfying Hypothesis 5.9. Then $F^{*}(H)$ is simple.

Proof. Let $L=\left\langle J^{L\langle H\rangle}\right\rangle$; by $L$-Balance, $L=L_{1} L_{1}^{a}$ where $L_{1}$ is a 2-component of $L(H)$. As $O(H)=1, L_{1}$ is a component. From 5.2(5), 


$$
K K^{a}=\left\langle J_{B}^{K K^{a}}\right\rangle \leqq L,
$$

as $K K^{a} \subseteq L(H)$ by $L$-Balance. If $L \neq L_{1}$, then $\left[K K^{a}, a\right]=K K^{a}$ forces $K K^{a}=L$ and $B \subseteq C_{H}(L)$. However we claim $C_{H}(L)=1$, whether or not $L=L_{1}$. As $O(H)=1$, it suffices to show $\left|C_{H}(L)\right|$ is odd. Pick $U \in \operatorname{Syl}_{2}(H)$ with $S \subseteq U$. If $B \leqq V \subseteq U$ with [KK $\left.K^{a}, V\right] \subseteq O\left(K K^{a}\right)$, then $V \subseteq N_{H}(B)$ implies $V=B$ by 5.2(6). Thus $B=C_{U}\left(K K^{a} / O\left(K K^{a}\right)\right)$, whence $C_{U}(L) \cong B$. By 5.2(8), $C_{U}(L) \cap C_{U}(L)^{x}=1$. As $x$ normalizes $J$, $L=L^{x}$ and we have $C_{U}(L)=1$. Because $L$ is subnormal in $H$, our claim is proved. But now $L=L_{1}$ and $C_{H}(L)=1$ imply that the lemma is valid.

By the argument of the preceding proof we have

Lemma 5.11. If $H$ satisfies Hypothesis 5.2, then

$$
B \in \operatorname{Syl}_{2}\left(C_{H}\left(K K^{a} / O\left(K K^{a}\right)\right)\right) \text {. }
$$

LeMma 5.12. Let $H$ satisfy Hypothesis 5.9. The following conditions hold:

(1) $\left\langle B, B^{x}\right\rangle=B \times B^{x}$;

(2) $B$ is isomorphic to a subgroup of $T / B$;

(3) If $|T: B|=2$, then $P$ is dihedral or semidihedral and $T=$ $\langle a, b\rangle$ where $B=\langle b\rangle$ has order 2. Further $[S, S] \subseteq D \times P,[S, S]$ is a direct product of two cyclic groups and $\langle a\rangle \subseteq \Omega_{1}([S, S]) \leqq\langle z, a\rangle$ where $z \in \operatorname{Inv}\left(C_{D}(S)\right)$.

Proof. By 5.9(3), $\left\langle B, B^{x}\right\rangle=B \times B^{x} . \quad$ As $B^{x} \subseteq T=Q \cap P,(2)$ holds and implies the first part of (3). By Lemmas 5.4 and 5.5, DE $\operatorname{Syl}_{2}(J)$ is dihedral. Pick cyclic groups $\langle d\rangle$ and $\langle p\rangle$ to be of maximum order in $D$ and $P$ respectively and normal in $S$. As $J / O(J) \cong L_{2}(q)$ or $A_{7}$, the structure of Aut $(J / O(J))$ forces $[S, S] \subseteq\langle d\rangle P$. As $S / C_{S}(\langle p\rangle)$ is abelian,

$$
[S, S] \subseteq\langle d\rangle P \cap C_{S}(\langle p\rangle) \subseteq\langle d\rangle C_{P}(\langle p\rangle) .
$$

If $|P| \geqq 8, C_{P}(\langle p\rangle)=\langle p\rangle$ and we are done, so assume $P=T=\langle a, b\rangle$. As $T \leqq S,[b, x]=a$ and $S=\langle x\rangle Q$ where $|S: Q|=2$. (Recall $Q=$ $N_{S}(B)$.) Consider $\bar{S}=S / D$; it suffices to show $[\bar{S}, \bar{S}] \subseteq\langle\bar{a}\rangle$. From knowledge of $J / O(J), \bar{S} / \bar{T}$ is isomorphic to a subgroup of $\boldsymbol{Z}_{2} \times \boldsymbol{Z}_{2^{m}}$. In particular $[\bar{S}, \bar{S}] \subseteq \bar{T}$. By $5.2(8), x^{2} \in T$, whence $x^{2} \in C_{T}(x)=\langle a\rangle$. Thus $\bar{x} \bar{T}$ is an involution and $S=\langle x\rangle Q$ implies

$$
\bar{S} / \bar{T}=\langle\bar{x}\rangle \bar{T} / \bar{T} \times \bar{Q} / \bar{T} .
$$

In particular $\bar{Q} / \bar{T}$ is cyclic, forcing $\bar{Q}$ to be abelian. As $\bar{x}$ acts as an automorphism of order 2 on $\bar{Q}, \bar{x}$ inverts $[\bar{Q}, \bar{x}]$. As $\bar{x}$ does not invert 
$\bar{b}$ or $\bar{b} \bar{a},[\bar{Q}, \bar{x}] \subseteq\langle\bar{a}\rangle$, and the desired conclusion follows.

Now we return to consideration of the possibilities for $K$.

Lemma 5.13. If $K=K^{a}$, then $K / O(K)$ is not isomorphic to $A_{7}$, $A_{9}$ or $A_{11}$.

Proof. Suppose the contrary. By Theorem 1.1 of [25], $G$ has no 2-subgroup, $B_{1}$, and 2-component, $K_{1}$, of $C_{G}\left(B_{1}\right)$ with $K_{1} / O\left(K_{1}\right) \cong$ $A_{2 n+1}$ for any $n \geqq 9$. In particular, $K / O(K) \cong A_{7}$. Recall that $T=$ $Q \cap P=N_{P}(B)$. As $|T: B|=2$ by Proposition $2.3(1),|B|=2$ by 5.12(2). By Lemmas 5.10 and 5.11, Theorem 1.5 is applicable to $\bar{G}_{0} / O\left(\bar{G}_{0}\right)$ and yields $L\left(G_{0}\right) / O_{2^{\prime} 2}\left(L\left(G_{0}\right)\right) \cong A_{9}$ or $H e$. As $K=K^{a}$, we have $J / O(J) \cong A_{5}$ by Lemma 5.4. Since $H e$ does not admit an action of $a$ with $L\left(C_{H e}(a)\right)$ having a component of type $A_{5}, L\left(G_{0}\right) / O_{2^{\prime} .2}\left(L\left(G_{0}\right)\right) \cong A_{9}$. Hence $L\left(G_{0}\right) / O\left(L\left(G_{0}\right)\right)$ is isomorphic to a covering group of $A_{9}$. As $O\left(L\left(G_{0}\right)\right) L\left(C_{G_{0}}(a)\right) / O\left(L\left(G_{0}\right)\right) \cong A_{5}$, we have $L\left(G_{0}\right) / O\left(L\left(G_{0}\right)\right) \cong A_{9}$, contradicting Theorem 1.1 of [25].

Lemma 5.14. The case $K / Z^{*}(K) \cong L_{3}(4)$ does not occur in $G$.

Proof. Assume the contrary. We have $J / O(J) \cong L_{2}(5)$ or $L_{2}(7)$ and by Proposition 2.3(1), $|T: B|=2$. By Lemma 5.8 and Hypothesis 5.9, Lemma 5.12 applies to $\bar{G}_{0}$, so it suffices to show that the assumption that there is a group $H$ satisfying the hypotheses of Lemma 5.12 with $K / Z^{*}(K) \cong L_{3}(4)$ leads to a contradiction. Assume the notation of Lemma 5.12 and Hypotheses 5.2 and 5.9.

By Lemma 5.4, $Z(K / O(K)) \neq 1$ and so $Z^{*}(K)=B O(K)$ by Lemma 5.11. As $|B|=2, m(K)=5>4=m(S)$ from Lemma 5.12. Thus $S<U \in \operatorname{Syl}_{2}(H)$ and we can find $u \in N_{U}(S)-S$ with $u^{2} \in S$. As $a^{u} \neq a$ and $\langle a\rangle=P \cap Z(S)$ by Lemma 5.5, $P^{u} \cap P=1$ and $P$ acts faithfully on $J / O(J)$. Since $P$ has maximal class, $P$ is dihedral of order $\leqq 16$. In particular, $J=L\left(C_{G}(a)\right)$.

By the structure of $L_{3}(4)$, there exists $g \in K$ with $a^{g} \in a z B$. By Lemma 5.7, $a^{g}=a z b$ where $\langle b\rangle=B$. Since $b^{x}=a b, b^{x g}=a z$. Let $Y=C_{H}(\langle a, z\rangle)$ and $K^{x g}=L\left(C_{G}(\alpha z)\right)$. Note that $S \in \operatorname{Syl}_{2}(Y)$. If $a$ acts as an inner automorphism on $K^{x g}$, then $Y \cap K^{x g}=C_{K^{x g}}(a)$ has 2-rank 5 , contrary to $m(S)=4$. Thus $a$ is outer on $K^{x g}$ whence $a \notin[S, S]$, contrary to Lemma $5.12(3)$.

Lemma 5.15. The case $K / O(K) \cong A_{10}$ does not occur in $G$.

Proof. In this case $T / B \cong E_{4}$ by Proposition $2.1(1)$, and as in Lemma 5.14 it suffices to show that no $H$ exists satisfying Hypothesis 5.9 with $K / O(K) \cong A_{10}$. 
Suppose such an $H$ exists. By Lemma $5.12(2) B$ is elementary abelian of order 2 or 4. By Hypothesis 5.9(3) either $x \in N_{P}(Q)$ and $B^{x} \leqq T=N_{P}(B)$ or $B \leqq P, B^{x} \supseteqq P$ and $P=T$. In either case $B \leqq T$, $B^{x} \leqq T$ and $B \cap B^{x}=1$ imply $T \cong E_{8}$ or $E_{16}$. Also $x$ normalizes $T$ in both cases.

We claim $a$ is not fused to any element of $B$, for if so, then for some $w \in H, a \in B^{w} \cong S$. Let $L=L\left(C_{H}\left(B^{w}\right)\right)=K^{w} . \quad L / O(L) \cong A_{10}$ and by Lemma $5.11, B^{w} \in \operatorname{Syl}_{2}\left(C_{H}(L / O(L))\right)$. But then as $J$ and $L$ are distinct components, $D=S \cap J \subseteq B^{w}$, whence $|D| \leqq 4$, not the case.

Now consider $N=N_{H}(T)$ and let $A=T \cap K \cong E_{4}$. Then $T=$ $A \times B$ with $N \cap K$ inducing an $S_{3}$ on $A$ and centralizing $B$. If $a \in A$, then $a \sim z$ in $K \subseteq C_{H}(B)$, contrary to Lemma 5.7. Thus $a \in T-A$. Further, $x$ acts on $T$ and $B \cap B^{x}=1$.

Suppose first that $A=A^{x}$; we see that $x$ normalizes $\langle A, a\rangle=$ $\langle A, b\rangle$ for some $b \in B$. But then all elements of $\langle A, a\rangle-A$ are conjugate under $\langle N \cap K, x)$, contrary to $a \not b$.

If $\left|A \cap A^{x}\right|=2$, then $A A^{x} \cong E_{8}$ and $\left|C_{A A^{x}}(x)\right|=4$. If $T=A A^{x}$, then $a \in C_{T}(x) \subseteq A A^{x}$, while if $T \cong E_{16}$, then $|B|=4$ and $T=B \times B^{x}$ implies $\left|C_{T}(x)\right|=4$ and again $a \in A A^{x}$. But $A A^{x}=A \times\langle b\rangle$ for some $b \in B$ and $\langle N \cap K, x\rangle$ acts as $\operatorname{GL}(3,2)$ on $A A^{x}$ contrary to $a \nsim b$.

Finally suppose $|B|=4$ and $T=A \times A^{x}$. If $A^{x}=B$, then $N_{H}(B)$ contains a 2-element acting nontrivially on $B$. By Hypotheses 5.2(4) $N_{H}(B)$ does not contain an element acting as an outer automorphism on $K / O(K)$, so by Lemma 5.11, $K B$ contains a Sylow 2-subgroup of $N_{H}(B)$. Thus no 2-element acts nontrivially on $B$, and $\left|A^{x} \cap B\right| \leqq 2$. If $A^{x} \cap B=2$, then $\langle N \cap K, x\rangle$ acts as $S_{5}$ on $T$ and every element of $T$ is fused to $B$ contrary to $a \nsim B$. Similarly if $A^{x} \cap B=1$, then all involutions of $T-B$ are fused by $\langle N \cap K, x\rangle$ and $B \cap B^{x} \neq 1$, again a contradiction.

LEMMA 5.16. The cases $K / Z^{*}(K) \cong L_{4}(q), K / Z^{*}(K) \cong U_{4}(q)$ and $K / Z^{*}(K) \cong \Omega^{-}\left(8, q^{1 / 2}\right)$ do not occur in $G$.

Proof. Suppose one of these cases occurs. By Lemma 5.4, $J / O(J) \cong L_{2}\left(q^{2}\right)$.

Consider first the possibility that $a$ acts as an outer automorphism on $K / O(K)$. By Proposition 2.3(3), $a \sim a z Z^{*}(K)$ in $N_{G}(B)$, so Lemma 5.3 implies $O(K) \subset Z^{*}(K)$. As $a$ is outer on $K / O(K)$, we have $|T: B|=2$. Passing to the section $\bar{G}_{0}$ of Lemma 5.8, it suffices to show that no group $H$ exists satisfying Hypothesis 5.9 with $K / Z^{*}(K) \cong L_{4}(q)$ or $U_{4}(q)$ or $\Omega^{-}\left(8, q^{1 / 2}\right)$, a outer on $K / Z^{*}(K)$ and $|T: B|=2$.

Suppose $H$ exists. By Propositions 2.7, 2.8 and 2.10 and Lemmas 5.11 and 5.12, $Z^{*}(K)=O(K) B$ with $B=\langle b\rangle$ cyclic. Further, as 
$|T: B|=2, B$ has order $2, P$ has maximal class and $a \sim a z b$ in $C_{H}(b)$. Thus $b \sim a b \sim a z$ in $H$. Consider $Y=C_{H}(\langle a, a z\rangle)$. Clearly $S \in \operatorname{Syl}_{2}(Y)$ and $S$ acts on $L=L\left(C_{I I}(\alpha z)\right) \cong K$. Now let $M=O^{2^{\prime}}\left(C_{L}(a)\right)=$ $O^{2^{\prime}}\left(C_{L\left(C_{H I}(a)\right)}(a z)\right)$. As $J \supseteqq C_{H}(a), C_{L}(a)$ acts on $J$ and a Sylow 2-subgroup of $O^{2}\left(C_{L}(a)\right)$ must centralize $J / O(J)$. Thus a Sylow 2-subgroup of $\langle M, a\rangle$ is isomorphic to a subgroup of $P$, a group of maximal class. Let $R \in \operatorname{Syl}_{2}(M)$. As $[R, a]=1$ and $\langle R, a\rangle$ is of maximal class, $a \in$ $Z(R)$. Thus $a \in Z^{*}(M)$ and $M / O(M) \cong \mathrm{SL}(2, r), r$ odd, or $\hat{A}_{7}$. By Propositions 2.7, 2.8 and 2.10 there is no involutory automorphism $\alpha$ of $L$ with $\alpha \in O^{2^{\prime}}\left(C_{L}(\alpha)\right) \cong \mathrm{SL}(2, r)$ or $\hat{A}_{7}$, a contradiction.

We have proved that $a$ does not act as an outer automorphism on $K / O(K)$. Thus $K / Z^{*}(K)$ is isomorphic to $L_{4}(q)$ with $q \equiv 3(\bmod 4)$ or to $U_{4}(q)$ with $q \equiv 1(\bmod 4)$. By Hypotheses 5.2(6) and the structure of $K, T \cap K$ is cyclic of order at least 4. Again it suffices to show that no $H$ exists satisfying Hypothesis 5.9 with $K$ and $a$ as above.

Suppose such an $H$ exists. As $J / O(J) \cong L_{2}\left(q^{2}\right), Z^{*}(K)=O(K)$. If $a \in K$, then $a \sim z$ in $K$ by Proposition 2.2, contrary to Lemma 5.3. Thus $a \in K B-B$. By Proposition 2.1, $T / B$ is cyclic; so by Lemma $5.12, B$ is cyclic. We have $K B=K \times B, T=(T \cap K) \times B$ and $\langle a b\rangle=$ $\Omega_{1}(T \cap K)$. As $\langle x\rangle$ normalizes $T=N_{P}(B)$ by $5.9(3), b^{x}=a b$ implies $B \cong T \cap K$.

From the structure of $K / O(K)$ we know $T \cap K \cong Z_{2^{s}}$ where $2^{s} \| q+\varepsilon$ with $\varepsilon=-1$ when $K / O(K) \cong L_{4}(q)$ and $\varepsilon=1$ when $K / O(K) \cong$ $U_{4}(q)$. If $S \notin \operatorname{Syl}_{2}(H)$, then we could find $U \in \operatorname{Syl}_{2}(H)$ with $S \subset U$ and $u \in N_{U^{\prime}}(S)-S$. As before $P^{u}$ would act faithfully on $J / O(J)$. But $T \cong \boldsymbol{Z}_{2^{s}} \times \mathbb{Z}_{2^{s}}$ cannot act faithfully on $L_{2}\left(q^{2}\right)$.

Thus $S \in \operatorname{Syl}_{2}(H)$. By Proposition 2.2(2), $(a b)^{g}=z$. As $b^{x}=a b$, $b^{x g}=z$. Consider the action of $S$ on $L=K^{x g} \unlhd C_{H}(z)$. By Propositions 2.7 and 2.8 and the congruences on $q$, the outer automorphism group of $L / O(L)$ is abelian. As $a \in[B, x] \subseteq[S, S], a$ is inner on $L / O(L)$. Let $L_{0}=O^{2^{\prime}}\left(C_{L}(a)\right)$. By Propositions 2.7 and 2.8, $L_{0} / O\left(L_{0}\right)$ is isomorphic to $\mathrm{SL}(2, q) * \mathrm{SL}(2, q)$ or to $L_{2}\left(q^{2}\right)$. As $z \in J$, we argue as before that either $\left[J, L_{0}\right] \subseteq O\left(C_{G}(a)\right)$ or $L_{0}$ is solvable and if $R_{0} \in \operatorname{Syl}_{2}\left(C_{G}\left(L_{0}\right)\right)$, then $\left[J, R_{0}\right] \subseteq O\left(C_{G}(a)\right)$. Thus either $\left[D, L_{0}\right] \subseteq O\left(L_{0}\right)$ or $\left[D, R_{0}\right]=1$ for some $R_{0} \in \operatorname{Syl}_{2}\left(C_{G}\left(L_{0}\right)\right)$. Since $B^{x g}=C_{S}(L / O(L))$ is cyclic with $\langle z\rangle=$ $\Omega_{1}\left(B^{x g}\right),\langle a, e\rangle$ acts faithfully on $L / O(L)$ for any $e \in \operatorname{Inv}(D-\langle z\rangle)$. From $\left[L_{0}, e\right] \subseteq O(L)$, we deduce by Propositions 2.7 and 2.8 that $L\left(C_{L / O(L)}(e)\right) \cong \operatorname{PSp}(4, q)$. But by $5.2(4), J_{B}$ projects to a standard component in $K / O(K)$, whence $N_{H}(B)$ does not contain an involution $f$ with $L\left(C_{K / o(K)}(f)\right) \cong \operatorname{PSp}(4, q)$. This contradicts the existence of $e$, completing the proof of Lemma 5.16.

We conclude this section by collecting the results of Lemmas 5.4, 5.5 and $5.13-5.16$. 
Lemma 5.17. The possibilities for $J / O(J)$ and $K K^{a} / O\left(K K^{a}\right)$ are as follows:
$\underline{J / O(J)}$
$K K^{a} / O\left(K K^{a}\right)$
(1) $A_{7}$ or $L_{2}(q), q$ odd, $q>3$
(1) A central product $\hat{A}_{7} * \hat{A}_{7}$ or
(2) $L_{2}(q), q$ odd, $q>3$ $\mathrm{SL}(2, q) * \mathrm{SL}(2, q)$
(2) $L_{2}\left(q^{2}\right)$ with no diagonal auto- morphism
(3) $A_{6}=L_{2}(9)$
(4) $L_{2}\left(q^{2}\right), q$ odd .
(3) $A_{8}$
(4) $\operatorname{PSp}(4, q)$.

An immediate corollary of Lemmas 5.11 and 5.17 and Proposition 2.3(1) is the following.

Corollary 5.18. $\quad N_{P}(B)=B \times\langle a\rangle$.

6. The choice of $B_{0}$. In this section, we do some technical refinement of the choice of $B_{0}$ which is useful in the remaining cases. We first pick $x_{0}$ as follows:

$$
\begin{array}{rlrl}
x_{0}=x & & \text { if } B \leqq P \\
x_{0} \in & \left(Z_{i}(S) \cap P\right)-N_{P}(B) & & \text { otherwise . } \\
& \text { with } i \text { minimum and } & \\
& x_{0}^{2} \in N_{P}(B) . &
\end{array}
$$

Assume henceforth that $B \nsubseteq P$. By Corollary 5.18, we have $N_{P}(B)=B \times\langle a\rangle$ and $\langle a\rangle=Z(S) \cap P$. Thus $i \geqq 2$ and if $i=2$, then $\left[B, x_{0}\right]=\langle a\rangle$. Suppose that $i>2$. Then $Z_{2}(S) \cap P \subseteq B \times\langle a\rangle$. As $\left[Z_{2}(S) \cap P, x\right] \subseteq\langle a\rangle$ and $B^{x} \cap B=1$, we have $Z_{2}(S) \cap P=\langle a, b\rangle$ with $b^{2}=1, b^{x}=a b$. Repeating this argument we see that $Z_{3}(S) \cap(\langle B, a\rangle)=$ $\langle a, b\rangle$. Thus $x_{0} \in Z_{3}(S) \cap P, x_{0}^{2} \in\langle a, b\rangle$ and $\left[B, x_{0}\right] \subseteq\langle a, b\rangle$.

Definition. If $i=2$, let $b=1$. If $i=3$, let $b$ be the element of $B^{\sharp} \cap Z_{2}(S)$ described above.

LEMMA 6.1. One of the following holds:

(1) $x_{0} \in Z_{2}(S) \cap P$ and $\left[B, x_{0}\right]=\langle a\rangle$.

(2) $x_{0} \in Z_{3}(S) \cap P, Z_{2}(S) \cap P=\langle a, b\rangle, b \in B_{0},\left[B, x_{0}\right] \cong\langle a, b\rangle$ and $\left|S: C_{S}(b)\right|=2$.

Proof. It remains only to show that $b \in B_{0}$. If not, then $b^{x_{0}}=$ $a b$ and $D=\left\langle b, x_{0}\right\rangle$ is dihedral of order 8. As $D \leqq S$ and $\langle a, b\rangle \leqq S$, every element of $S$ induces an inner automorphism on $D$. Thus $S$ is a central product $D * C_{S}(D)$. If $P \cap C_{S}(D) \neq\langle a\rangle$, then as $P \leqq S, Z_{2}(S) \cap$ $P \cap C_{S}(D) \nsubseteq\langle a\rangle$, contrary to fact. Thus $P=D$ and $x_{0} \in Z_{2}(S) \cap P$. 
We would like to have $(*)$

$$
N_{S}\left(B_{0}\right) \in \operatorname{Syl}_{2}\left(N_{G}\left(B_{0}\right) \cap C_{G}\left(\langle a\rangle\left[B, x_{0}\right]\right)\right) .
$$

Notation. Set $E=\langle a\rangle\left[B, x_{0}\right]$. Note that $E=\langle a\rangle$ or $E=\langle a, b\rangle$.

Assume that $\left(^{*}\right)$ does not hold and let $T \in \operatorname{Syl}_{2}\left(N_{G}\left(B_{0}\right) \cap C_{G}(E)\right)$ with $N_{S}\left(B_{0}\right) \subseteq T$. Note $[S, b] \subseteq\langle a\rangle$, so $\left[N_{S}\left(B_{0}\right), b\right] \subseteq\langle a\rangle \cap B_{0}=1$. Pick $g \in C_{G}(E)$ so that $T^{g} \subseteq C_{S}(E) \in \operatorname{Syl}_{2}\left(C_{G}(E)\right)$. Replace $B$ by $B^{g}, x_{0}$ by $x_{0}^{g}, B_{0}$ by $B_{0}^{g}$. Since $J \leqq C_{G}(a), J_{E} \supseteqq C_{G}(E)$ and $C_{G}\left(J_{E} / O\left(J_{E}\right)\right) \cap C_{G}(E) \leqq$ $C_{G}(E)$. Thus, as $g \in C_{G}(E), \quad B^{g} \cong C_{S}\left(J_{E} / O\left(J_{E}\right)\right) \cap C_{S}(E)=C_{P}(E)$. It follows that $B^{g}$ satisfies the conclusions of Lemma 3.6. In particular, $N_{S}(B) \subseteq N_{S}\left(B_{0}\right)$, so $\left|N_{S}\left(B^{g}\right)\right|=\left|N_{S}(B)\right|$. We have proved the following.

Proposition 6.2. We may choose $B, x_{0}$ and $B_{0}$ in such a way that the following hold:

(1) Hypothesis 5.2 holds for $G$.

(2) Hypothesis 5.9 holds for $\bar{G}_{0}$.

(3) $N_{S}\left(B_{0}\right) \in \operatorname{Syl}_{2}\left(N_{G}\left(B_{0}\right) \cap C_{G}(E)\right)$, where $E=\langle a\rangle\left[B, x_{0}\right]$.

(4) Either $\left[B, x_{0}\right]=\langle a\rangle$ or $\left[B, x_{0}\right]=\langle a, b\rangle$ with $b \in C_{B}\left(x_{0}\right),\langle a, b\rangle=$ $Z_{2}(S) \cap P$.

7. The cases $K / O(K) \cong L_{2}\left(q^{2}\right)$ and $K \neq K^{a}$. In this section we obtain the following reduction of Theorem $B$.

Lemma 7.1. The cases $K / O(K) \cong L_{2}\left(q^{2}\right)$ and $K \neq K^{a}$ do not occur in $G$.

Combining this with Lemma 5.17, we have the following immediate corollary.

CoRollary 7.2. $J / O(J) \cong L_{2}\left(q^{2}\right)$ and either $K / O(K) \cong \operatorname{PSp}(4, q)$ or $q=3$ and $K / O(K) \cong A_{8}$.

We continue the notation and hypotheses of $\S 5$. Moreover we assume throughout this section that eithen $K / O(K) \cong L_{2}\left(q^{2}\right)$ or $K \neq$ $K^{a}$. We prove Lemma 7.1 via a sequence of reductions.

$$
|B|>2 \text {. }
$$

Proof. Assume that $|B|=2$. If $K / O(K) \cong L_{2}\left(q^{2}\right)$, then by Lemma 5.11 , one of the conclusions of Theorem B holds. If $K \neq K^{a}$, then $m(K)=1$ by Lemma 5.4 and $F^{*}(G)$ is known by Theorem 1.3. Again Theorem B holds. 
(2) $B_{0} \neq 1 . \quad \bar{B}=\left\langle\bar{b}_{1}\right\rangle$ has order 2. $F^{*}\left(\bar{G}_{0}\right) / O\left(F^{*}\left(\bar{G}_{0}\right)\right)$ is isomorphic to $\operatorname{PSp}(4, q)$.

Proof. We have $|T: B|=2$; so $B \cap B^{x}=1$ implies $T \subset P$. Thus $\bar{G}_{0}$ is proper, $B_{0} \neq 1$ and $\bar{B}=\left\langle\bar{b}_{1}\right\rangle$ has order 2 . If $K / O(K) \cong L_{2}\left(q^{2}\right)$, Lemma 5.11 and the conditions of Lemma 5.8 imply that $\bar{K}$ is a standard component in $\bar{G}_{0}$. Now $\left|P_{0}: B_{0}\right| \geqq 8$ and by Lemma 5.12(3), $\bar{P}_{0}$ has maximal class. By Lemma 5.8, $\bar{P}_{0}$ contains a Sylow 2-subgroup of $C_{F^{*}\left(\bar{G}_{0}\right)}(\bar{a}) \cap C_{F^{*}\left(\bar{G}_{0}\right)}\left(\bar{J}_{0} / O\left(\bar{J}_{0}\right)\right)$. By the hypotheses of Theorem B, if $K / O(K) \cong L_{2}\left(q^{2}\right)$, then $F^{*}\left(\bar{G}_{0}\right) / O\left(F^{*}\left(\bar{G}_{0}\right)\right)$ is simple of restricted type and we conclude

$$
F^{*}\left(\bar{G}_{0}\right) / O\left(F^{*}\left(\bar{G}_{0}\right)\right) \cong \operatorname{PSp}(4, q) .
$$

If $K \neq K^{a}$, then $m(K)=1$ and Theorem 1.3 lead to the same conclusion.

(3) We may assume that $K \neq K^{a}$.

Proof. Assume $K / O(K) \cong L_{2}\left(q^{2}\right)$. We shall find $B_{1} \cong P_{0}$ satisfying the conclusions of Lemma 3.6 and with $\left\langle\left(J_{B_{1}}\right)^{L\left(G_{\left.G^{(} B_{1}\right)}\right)}\right\rangle=K_{1} K_{1}^{a}$ with $K_{1}$ of 2-rank 1.

By Lemma 5.12(3) and the structure of Aut PSp $(4, q)$, it is clear that

$$
P_{0} \in \operatorname{Syl}_{2}\left(C_{\bar{G}_{0}}(\bar{a}) \cap C_{\bar{G}_{0}}\left(\bar{J}_{0} / O\left(\bar{J}_{0}\right)\right)\right) .
$$

Further our knowledge of $\operatorname{PSp}(4, q)$ tells us that $\bar{P}_{0}$ contains an involution $\bar{b}_{2}$ which is 2-central in $\bar{G}_{0}$. Pick $b_{2} \in P_{0}$ so that $b_{2}$ projects to $\bar{b}_{2}$ and define $B_{2}=\left\langle b_{2}, B\right\rangle$. Clearly

$(* *) \quad\left[J, B_{2}\right] \cong O(J)$, and $L\left(C_{G}\left(B_{2}\right)\right) / O\left(L\left(C_{G}\left(B_{2}\right)\right) \cong \mathrm{SL}(2, q) * \mathrm{SL}(2, q)\right.$.

Let $Q_{2}=N_{S_{0}}\left(B_{2}\right)$. As $\left\langle\bar{b}_{1}, \bar{b}_{2}\right\rangle \subseteq \bar{P}_{0}$ and $\bar{P}_{0}$ is of maximal class (with $\left.\langle\bar{a}\rangle=Z\left(\bar{P}_{0}\right)\right),\left|\bar{S}_{0}: C_{\bar{S}_{0}}\left(\bar{b}_{2}\right)\right|=\left|\bar{S}_{0}: C_{\bar{S}_{0}}\left(\bar{b}_{1}\right)\right|$, whence $\left|Q_{1}\right|=|Q|$. We claim $(* * *)$

$$
Q_{2} \in \operatorname{Syl}_{2}\left(N_{G}\left(B_{2}\right) \cap N_{G}\left(J_{B_{2}} O\left(C_{G}\left(B_{2}\right)\right)\right)\right) .
$$

If not, apply Lemma 3.3 to find a conjugate, $B_{3}$, of $B_{2}$ with $B_{3} \leqq P$ and $\left|N_{S}\left(B_{3}\right)\right|>\left|Q_{2}\right|=|Q|$. By Lemma 3.6(4) applied to $B, J_{B_{3}}$ must correspond isomorphically to a 2-component of $N_{G}\left(B_{3}\right)$, not the case. Thus (***) holds and as $Q_{2} \subseteq G_{0}$, the structure of $\operatorname{PSp}(4, q)$ implies $(* * * *)$

$$
B_{2} \in \operatorname{Syl}_{2}\left(C_{G}\left(L\left(C_{G}\left(B_{2}\right)\right)\right) / O\left(L\left(C_{G}\left(B_{2}\right)\right)\right)\right) .
$$

Finally the conclusions of Lemma 3.6 follow easily from $\left(^{* *}\right),\left(^{* *}\right)$ and $(* * * *)$.

Notation. Let $L=L\left(C_{G}\left(B_{0}\right)\right)$. Note that by Proposition 6.2, 
$S \cap L \in \operatorname{Syl}_{2}\left(C_{L}(a)\right)$.

$$
N_{G}(S) \subseteq C_{G}(a)
$$

Proof. Suppose that $N_{G}(S) \nsubseteq C_{G}(a)$. We have $C_{P}(S)=\langle a\rangle$ by Lemma 5.5 and $C_{L}(a)$ contains an involution acting on $L\left(C_{L / O(L)}(a)\right)$ and hence on $J / O(J)$ as an outer diagonal automorphism. Accordingly, $Z(S)=\langle z, a\rangle$ and $a^{u} \in\{z, a z\}$, for some $u \in N_{G}(S)$. Then $(P \cap Z(S)) \cap$ $\left(P^{u} \cap Z(S)\right)=\langle a\rangle \cap\left\langle a^{u}\right\rangle=1$. So $P \cap P^{u}=1$ and $P^{u}$ acts faithfully on $J / O(J)$. Consequently $P$ is isomorphic to a subgroup of $\operatorname{Aut}\left(L_{2}(q)\right)$. As $N_{S}\left(B_{0}\right)$ contains a Sylow 2-subgroup of $C_{L}(a)$ we may see in $L$ that $N_{P}\left(B_{0}\right) \supseteqq D_{1} \times B_{0}$ with $D_{1} \cong D$. The structure of Aut $\left(L_{2}(q)\right)$ forces $\left|B_{0}\right|=2$ and $D \subseteq P P^{u}$.

Let $F=J_{e}(S)$. As $P P^{u}=P \times P^{u}, m(S)=6$ and $F \subseteq P P^{u}$. Likewise $D \times D^{u} \subseteq F$. Let $B_{0}=\langle b\rangle$. Since $b^{u}$ acts on $J / O(J)$ as a field automorphism, $|D| \geqq 8$. We have $F=D \times\left\langle b^{u}\right\rangle \times D^{u} \times\langle b\rangle$. If $a$ is fused in $G$ to $a z$, then we may take $a^{u}=a z$, whence $P^{u} \cap D=1$, $P^{u}$ centralizes $D$ and by the structure of Aut $\left(L_{2}(q)\right),\left|P^{u}\right| \leqq 4$. As $|P| \geqq|D|\left|B_{0}\right| \geqq 16$, we see that $a$ is not $G$-conjugate to az. Thus $a^{u}=z$ and $u^{2} \in S$.

Pick $U \in \operatorname{Syl}_{2}(G)$ with $\langle S, u\rangle \subseteq U$ and $N_{U}(F) \in \operatorname{Syl}_{2}\left(N_{G}(F)\right)$. Our conditions imply $\langle a, z\rangle=\Omega_{1}([F, F])$, whence $\left|N_{U}(F): S\right|=2$. It follows easily that $F=J_{e}\left(N_{U}(F)\right)$ and $|U: S|=2$. Consequently fusion in $Z(F)=\left\langle a, z, b, b^{u}\right\rangle$ is controlled in $N_{G}(F)$. We have $a^{u}=z, b^{x}=a b$ and $b^{u x}=b^{u} z$. The last equation holds because first $\left[b^{u}, x\right] \in z P$ by properties of Aut $\left(L_{2}(q)\right)$ and second, $\left[b^{u}, x\right] \in P^{u} \cap Z(F)=\left\langle z, b^{u}\right\rangle$. So far we have the following fusion information:

(A) $a^{G} \cap Z(F)=\{a, z\}$.

(B) $(a z)^{G} \cap Z(F)=\{a z\}$.

(C) $b \sim b^{u} \sim b^{u} z \sim a b$.

(D) $b a z \sim b^{u} a z \sim b^{u} a \sim b z$.

(E) $b b^{u} \sim b b^{u} a z$ and $b b^{u} a \sim b b^{u} z$.

Pick $R \in \operatorname{Syl}_{2}\left(C_{G}(b)\right)$ with $C_{S}(b) \subseteq R$. Since $a \in[F, F]$ and $F \subseteq C_{S}(b) \leqq$ $R, a \in R^{\prime} \cong L$ and $Z(R)=\langle a z, b\rangle . \quad Z(U)=\langle a z\rangle$, so $b$ is not 2-central in $G$ and $R \subset V \in \operatorname{Syl}_{2}(G)$. Let $v \in N_{V}(R)-R$. As $F \leqq V, x$ centralizes $\langle a z\rangle=[F, F] \cap Z(R)$ and we have $b^{v}=b a z$. Thus $C_{b}=\left\{b^{G}\right\} \cap Z(F)$ contains at least 8 involutions. If $\left|C_{b}\right|=8$, then $\left|U: C_{U}(b)\right|=8$. But $b^{u}$ acts on $J / O(J)$ as a field automorphism, and $\left[S, b^{u}\right] \subseteq P^{u}$ implies $\left|S: C_{S}\left(b^{u}\right)\right|=2$. Thus $\left|U: C_{U}(b)\right|=\left|U: C_{U}\left(b^{u}\right)\right|=4$ and $\left|C_{b}\right|>8$. Our conditions imply $\left|C_{b}\right|=10$ or 12 . As $N_{G}(F)$ normalizes the series $1 \subseteq$ $[F, F] \subseteq Z(F)$, no element of order 5 can act nontrivially on $Z(F)$, whence $\left|C_{b}\right|=12$ and

$$
b^{G} \cap Z(F)=Z(F)-\langle a, z\rangle .
$$


Let $N=N_{G}(F)$ and $\bar{N}=N / C_{N}(Z(F)) . \quad N$ normalizes the series $1 \subseteq$ $\langle a z\rangle \subseteq\langle a, z\rangle \subseteq Z(F)$ and we have $\bar{S}=O_{2}(\bar{N}), \bar{N} / \bar{S} \cong S_{3}$. Suppose $\lambda \in N$ with $|\bar{\lambda}|=3$ and $|\lambda|=3^{m}$ for some $m \geqq 1$. Clearly $\lambda$ centralizes $a$, and it follows that $\lambda$ acts on $J$ and normalizes $D=F \cap J$ and $F \cap P=$ $C_{F}(J / O(J))$. But then $\lambda$ normalizes $\langle a, b\rangle=Z(F) \cap P$, whence $\left|b^{N}\right|=$ 4 , a contradiction.

$$
Z_{2}(S) \cap P=\langle a, b\rangle, \quad x_{0} \in Z_{3}(S) \cap P, \quad b \in B_{0} .
$$

Proof. If not, then by $\S 6, x_{0} \in Z_{2}(S)$ and $\left[x_{0}, B\right]=\langle a\rangle,\left[x_{0}, B_{0}\right] \subseteq$ $B_{0} \cap\langle a\rangle=1$. Then $B=\left(B \cap K K^{a}\right) \times B_{0}$ with $B \cap K K^{a} \subseteq L$. But then $\langle a\rangle=\left[B \cap K K^{a}, x_{0}\right] \subseteq L$ and $a \sim z$ in $L$. Then $a \sim z$ in $N_{G}(S)$, not the case.

$$
a b \in L \text { and } a b \sim z \text { in } C_{G}\left(B_{0}\right) .
$$

Proof. $\left[B \cap K K^{a}, x_{0}\right] \subseteq L \cap\langle a, b\rangle . \quad$ As $b \in B_{0}, b \in L$. The argument in (5) shows $a \notin L$. Thus $a b \in L$ and $a b \sim z$ in $L$.

Notation. $U=C_{S}(a b), N=N_{G}(Z(U)), C=C_{N}(Z(U))$.

$$
Z(U)=\langle z, a, b\rangle \text {. }
$$

Proof. We have $|S: U|=2$ and $N_{S}\left(B_{0}\right)$ normalizes $B_{0} \cap U=\langle b\rangle$, so $N_{S}\left(B_{0}\right) \subseteq U . \quad N_{S}\left(B_{0}\right)$ contains a 2-element acting as an outer diagonal automorphism on $J / O(J)$, so $Z(U) \subseteq\langle z\rangle P$ where $\langle z\rangle=C_{S}(D)$. Also $Z(S)=C_{Z(U)}(x)=\langle z, a\rangle$, forcing $Z(U)=\langle z, a, b\rangle$.

(8) $N / C \cong S_{3}$. The $G$-classes of $Z(U)$ are:

$$
a \sim z b \sim a z b ; \quad b \sim a b \sim z ; a z \text {. }
$$

Proof. First, by the structure of $\operatorname{PSp}(4, q),\langle a b, z\rangle$ is normal in a Sylow 2-subgroup; $R$, of $L$. (Note that $L / O(L) \cong \operatorname{PSp}(4, q)$ not $\mathrm{Sp}(4, q)$ because $K K^{a} / O\left(K K^{a}\right) \cong \mathrm{SL}(2, q)^{*} \mathrm{SL}(2, q)$.) $\quad$ Further, for some $r \in \operatorname{Inv}(R),(a b)^{r}=z$, whence $r$ normalizes $\langle a b, z\rangle$ and centralizes $b \in B_{0}$. Thus $r \in N$ and $(a b)^{r}=z$. Now $S \subseteq N$ and $|S: S \cap C|=|S: U|=2$, so $|N: C|_{2}=2$. Further $N_{G}(S) \subseteq C_{G}(a)$ and the structure of $C_{G}(a)$ imply that none of the involutions in $Z(S)$ are G-conjugate. Thus $N=\langle r, x, C\rangle, N / C \cong S_{3}$ and the $G$-classes of $Z(U)$ are as described.

(9) $Z(U)$ has at most two $G$-classes of involutions.

Proof. As $C \leqq C_{G}(\langle a, z\rangle), C$ acts on $J$ and centralizes $z$. It follows that $C / C_{C}(J / O(J))$ has a normal 2-complement. Further if $X$ is the largest subgroup of $C_{C}(J / O(J))$ normal in $N$, then $C_{C}(J / O(J)) \cap Z(U)=$ $\langle a, b\rangle$ forces $X \cap Z(U)=1$, whence $X \subseteq O(C)$. We conclude that $C=$ 
$U O(C)$ and $U$ acts faithfully on a direct product of 3 copies of $J / O(J)$. Roughly speaking each pair of copies generates a $\operatorname{PSp}(4, q)$.

Let $N_{1}=N_{G}(U)$. Clearly $N_{1}$ covers $N / O(C)$ and $x \in N_{1}$. Further $r w=g \in N_{1}$ for some $w \in O(C)$. As $g$ centralizes $B_{0} O(C) / O(C)$ and normalizes $U,\langle g, r\rangle$ centralizes $B_{0}$ and acts on $L$. Also $g^{2}$ centralizes $U$ and we can find a conjugate, $y$, of $g$ such that $\langle g, y\rangle$ acts on $U$ as a dihedral group of order 6 . In particular, $\langle g, y\rangle$ covers $N / C$ and we may assume that $[y, a]=1$.

It follows from $y \in C_{G}(a)$ that $D=D^{y}$ whence $\left|N_{1}: N_{N_{1}}(D)\right|=3$. As $D \cap Z(U)=\langle z\rangle$ and $D^{g} \cap Z(U)=\langle a b), D D^{g}$ is a direct product. Let $P_{1}=D D^{g} \cap P$. As $D D^{g} \subseteq L, b \notin D D^{g}$, whence $D D^{g} \cap D^{g y}=1$ and $E=D D^{g} D^{g y}=D \times D^{g} \times D^{g y}$. Looking in $\operatorname{PSp}(4, q)$ we see that $D D^{g}=D \times P_{1}$ with $\langle a b\rangle=P_{1} \cap Z(U)$. Since $P_{1} \leqq U$ and $(a b)^{y}=b$, $P_{1} P_{1}^{y}=P_{1} \times P_{1}^{y} \subseteq P \cap E$, as $y$ normalizes $P \cap U$. Thus $|P \cap U| \geqq\left|P_{1}\right|^{2}=$ $|D|^{2}$ and as $D P=D \times P$, we have $E=D \times(P \cap E)$. We claim $B_{1}=$ $(P \cap E) \cap(P \cap E)^{g} \subseteq B_{0}$. Indeed, since $\left[B_{0}, g\right]=1, B_{0} \subseteq(P \cap U) \cap(P \cap U)^{g}$. Suppose $B_{0} \leqq V \leqq(P \cap U) \cap(P \cap U)^{g}$. Then $V$ centralizes $J / O(J)$ and $J^{g} / O\left(J^{g}\right)$. Consequently $V$ centralizes $J_{B_{0}} / O\left(J_{B_{0}}\right)$ and $J_{B_{0}}^{g} / O\left(J_{B_{0}}^{g}\right)$. But

$$
\left\langle J_{B_{0}} O(L) / O(L), \quad J_{B_{0}}^{g} O(L) / O(L)\right\rangle=L / O(L) .
$$

It is immediate from Lemma 5.11 that $B_{0} \in \operatorname{Syl}_{2}\left(C_{G}(L / O(L))\right)$ and we conclude that $B_{0}=V$, whence $(P \cap U) \cap(P \cap U)^{g} \subseteq B_{0}$ and our claim is valid.

Now $\left|B: B_{1}\right| \leqq|D|^{2}$ and $D \times D^{g} \leqq L$ force $E=D \times D^{g} \times B_{1}$ with $D \cong E / D D^{g} \cong B_{1}$. Then $D D^{g} \cong L$ implies $B_{1}=E \cap B_{0}$. As $B_{1} \supseteqq U$ and $\left[g, B_{1}\right]=1$, we have $\left|N_{1}: N_{N_{1}}\left(B_{1}\right)\right|=1$ or 3 . As $B \cap Z(U)=\langle b\rangle$, $B_{1} B_{1}^{y}=B_{1} \times B_{1}^{y}$ and $\left|N_{1}: N_{N_{1}}\left(B_{1}\right)\right|=3$. Now $B_{1} B_{1}^{y} \subseteq P$, so $E=D \times$ $(E \cap P)=D \times B_{1} \times B_{1}^{y}$. Also $P_{1} \subseteq B_{1} B_{1}^{y}$ and $D D^{g}=P_{1} P_{1}^{g}$ imply $D \subseteq$ $\left\langle B_{1}^{\langle y, g\rangle}\right\rangle$. We conclude

$$
E=B_{1} \times B_{1}^{y} \times B_{1}^{y g} .
$$

Pick a foursgroup $A_{1}$ in $B_{1}$. Since $\left[B_{1}, g\right]=1$, we have

$$
A=\left\langle A_{1}^{\langle y, g\rangle}\right\rangle=A_{1} \times A_{1}^{y} \times A_{1}^{y g} \cong E_{64} .
$$

Consequently $A \cap L=A \cap D D^{g} \cong E_{16}$ and, looking in $\operatorname{PSp}(4, q)$, we see that $N_{L}\left(A \cap D D^{g}\right)=N_{L}(A)$ acts as $A_{5}$ or $S_{5}$ on $A \cap D D^{g}$ and centralizes $A_{1}$. More precisely we have:

(a) $A \cap D D^{g} \cap P=D D^{g} \cap(A \cap P)=D D^{g} \cap A_{1} A_{1}^{y}=A_{1}^{g}$.

(b) Every involution in $D D^{g}$ is $N_{L}(A)$-fused into $A_{1}^{y}$.

(c) $A_{1}^{y}$ contains two $L$-classes of involutions.

Repeating this argument with $A_{1}^{y} A_{1}^{y g}$ in place of $A_{1} A_{1}^{y}$, we see that all the involutions in $A$ are $N_{L}(A)$-fused into $A_{1}^{y g}$, whence $A$ intersects 
at most $2 G$-classes of involutions. As $Z(U) \subseteq A$, we are done.

Since (8) and (9) contradict each other, we have proved Lemma 7.1 .

8. The case $K / O(K) \cong \operatorname{PSp}(4, q)$ or $A_{8}$. In this section we handle the remaining possibilities for $K$ and thus complete the proof of Theorem B. In Lemma 8.1, we determine the structure of $\bar{G}_{0}$ in the case when $|P| \geqq 8$. In particular, this determines $G$ if $|P| \geqq 8$ and $|B|=2$, since $G=\bar{G}_{0}$ if $|B|=2$. In Lemma 8.2 , we use this information to completely eliminate the case $|P| \geqq 8$ and in Lemma 8.3 we treat the case $|P|=4$.

Lemma 8.1. Suppose that $|P| \geqq 8$. Then $F^{*}\left(\bar{G}_{0} / O\left(\bar{G}_{0}\right)\right) \cong A_{10}, L_{4}(q)$ or $U_{4}(q)$.

Proof. For simplicity of notation, we shall write $H=\bar{G}_{0}$ and use the notation of Hypothesis 5.9. Our first task will be to determine a Sylow 2-subgroup of $H$. By Lemma 5.12, $T \cong E_{4}$ and $P$ has maximal class and $[S, S]$ is abelian. Looking in $K$, we see that $S \notin \operatorname{Syl}_{2}(H)$. Since $J / O(J) \cong A_{6}$ or $L_{2}\left(q^{2}\right)$, we must have $Z^{*}(K)=O(K)$. Let $F=$ $J_{e}(S)$ and pick $U \in \operatorname{Syl}_{2}(H)$ with $S \subset U$ and $N_{U}(F) \in \operatorname{Syl}_{2}\left(N_{H}(F)\right)$.

$$
|U: S|=2 \text {. }
$$

Proof. Let $B=\langle b\rangle$. As we have noted in similar circumstances above, $Q \in \operatorname{Syl}_{2}\left(C_{H}(\langle a, b\rangle)\right)$. Looking in $K$ we see that $Q_{1}=Q \cap K\langle a, b\rangle=$ $D \times\langle\tau\rangle \times\langle a\rangle \times\langle b\rangle$, where $\tau$ is a non-2-central involution of $K$ and $\tau$ acts as a transposition or field automorphism on $J / O(J)$. For later use we record the existence of $g \in N_{K}(Q)$ with $a^{g}=a \tau,(a \tau)^{g}=a$.

Returning to $S$, we have $m(S)=5$ and $F=D \times\langle\tau\rangle(F \cap P)$. Since $\langle a, b\rangle \subseteq C_{P}(\tau)$, either $\tau$ or $\tau b$ centralizes a cyclic subgroup of order at least 4 in $P$. Thus $|F \cap P| \geqq 8$, and as $F \cap P \leqq P,|P: F \cap P| \leqq 2$. Consequently $\Omega_{1}([F, F])=\Omega_{1}([S, S])=\langle a, z\rangle$. (Note that $|D| \geqq 8$.) From $\langle a, z\rangle \leqq N_{U}(F)$, we deduce $\left|N_{U}(F): S\right|=2$. We claim $F=J_{e}\left(N_{U}(F)\right)$. If not we could find $A \cong E_{32}$ with $x \in A, v \in N_{U}(F)-S$. Since $P \cap$ $P^{v}=1, A \cap P=1$ and $A \cap S$ acts faithfully on $J / O(J)$. But this is impossible because $m(A \cap S)=4$. Thus $F=J_{e}\left(N_{U}(F)\right)$, whence $|U: S|=2$.

(2) $Z(F)=\langle a, z, \tau b\rangle$. We can divide $Z(Q)^{\sharp}$ into the following sets of $H$-conjugates:

$$
\begin{array}{ll}
C_{1}=a^{H} \cap Z(Q): & z \stackrel{v}{\sim} a \stackrel{g}{\sim} a \tau \stackrel{x}{\sim} \tau \\
C_{2}=(a z)^{H} \cap Z(Q): & a z \stackrel{g}{\sim} a z \tau \stackrel{x}{\sim} z \tau \\
C_{3}: & a \tau b \stackrel{g}{\sim} a b \stackrel{x}{\sim} b
\end{array}
$$




$$
\begin{array}{ll}
C_{4}: & a \tau b z \stackrel{g}{\sim} a b z \stackrel{x}{\sim} b z \sim \tau b \\
C_{5}: & \tau b z .
\end{array}
$$

Proof. Picking $v \in U-S$, we have $a^{v}=z$ or $a^{v}=a z$. In the latter case, $P^{v} \cap P=1=P^{v} \cap D$, whence $P^{v}$ acts faithfully on $J / O(J)$ and centralizes $D$, forcing $\left|P^{v}\right| \leqq 4$. Thus $a^{v}=z$. As $P^{v}$ still acts faithfully on $J / O(J)$ and has maximal class, $\left|P^{v}\right|=|P| \leqq 2|D|$. Further $a z$ is 2-central in $H$.

Let $\langle p\rangle$ be the cyclic subgroup of index 2 in $P$ and consider how the fours group $\langle\tau, b\rangle$ acts on $\langle p\rangle$. If $\langle\tau, b\rangle$ acts unfaithfully, then $F=D \times J_{e}(\langle\tau\rangle P)=D \times\langle\tau\rangle \Omega_{1}(P)$, and $Z(F)=\langle z, \sigma, a\rangle$ with $\sigma \in\{\tau, \tau b\}$. If $\langle\tau, b\rangle$ acts faithfully, $F=D \times\langle\tau, b\rangle\left\langle p^{2}\right\rangle$ and again $Z(F)=\langle z, \sigma, a\rangle$ with $\sigma \in\{\tau, \tau b\}$. As $N_{H}(F)$ controls fusion in $Z(F)$ and $Z(F) \cap[F, F]=$ $\langle z, a\rangle$, we see

$\left(^{*}\right) \quad a^{I I} \cap Z(F)=\{a, b\} ; \quad(a z)^{H} \cap Z(F)=\{a z\}$.

Consider the action of $\langle x, g\rangle$ on $Z(Q)=\langle z, \tau, a, b\rangle$. As $\langle z\rangle=$ $\Omega_{1}([Q, Q])$, we have

$$
z^{g}=z, \quad b^{g}=b, \quad a^{g}=a \tau, \quad(a \tau)^{g}=a .
$$

In particular, $a \tau \sim a$ implies $a \tau \notin Z(F)$, whence $\sigma=\tau b$. Since $\tau$ normalizes $N_{P}(\langle a, b\rangle)=\langle a, b, x\rangle$ and $\langle a, b, x\rangle \cong D_{8}$, it follows that $x \in F$ and $[x, \sigma]=1$. We have

$$
z^{x}=z, \quad b^{x}=b a, \quad a^{x}=a, \quad \tau^{x}=\tau a .
$$

Since $\sigma=\tau b \in Z(F), a \tau b \in Z(F)$ and we can pick $R \in \operatorname{Syl}_{2}\left(C_{H}(a \tau b)\right)$ with $F \subset R$. As $a \tau b \sim b$ and $\langle b\rangle \in \operatorname{Syl}_{2}\left(C_{H}(K / O(K))\right)$, we know that $Z(R) \cong E_{4}$. Thus $\langle a z\rangle=C_{F}(R)$ and $a \sim z$ in $R$. Consequently

$$
\tau b=a(a \tau b) \sim z a \tau b
$$

in $R$. This gives all of the desired fusion. Since $C_{3}$ through $C_{5}$ have representatives in $Z(F)$, we have $a^{H} \cap Z(Q)=C_{1},(a z)^{H} \cap Z(Q)=C_{2}$.

$$
C_{3} \nsim C_{4} \text {. }
$$

Proof. Suppose that $C_{3} \sim C_{4}$; then $b \sim b z$. Let $Y=C_{H}(\langle a, b z\rangle)$. We will show that $Y / O(Y)$ is a 2 -group, contradicting any possible action of $a$ on $L\left(C_{H}(b z)\right) \cong K$.

If $L\left(C_{H}(a)\right) \neq J$, then as $P$ has maximal class, Lemma 5.6 implies that $L\left(C_{H}(a)\right)$ contains a single additional 2-component, $L$, with $L / O(L)$ isomorphic to $\hat{A}_{7}$ or $\operatorname{SL}\left(2, q_{1}\right)$ for some odd prime $q_{1}$. Further $a \in$ $\mathrm{Syl}_{2}\left(Z^{*}(L)\right)$ and $P$ is semidihedral with $\langle a\rangle=C_{P}(L / O(L))$. Now $T=$ $N_{P}(\langle b\rangle) \cong E_{4}$ implies $b$ acts as an outer diagonal automorphism on 
$L / O(L)$. We have

$$
C_{J L / O(J L)}(b z)=C_{J O(J L) / O(J L)}(z) \times C_{L O(J L) / O(J L)}(b)
$$

is a product of two dihedral groups. As $J L O\left(C_{H}(a)\right) / O\left(C_{H}(a)\right)$ is selfcentralizing in $C_{H}(a) / O\left(C_{H}(a)\right)$, it follows from the structure of Aut $L_{2}(q)$ that $Y / O(Y)$ is a 2-group.

If $L\left(C_{H}(a)\right)=J, P$ contains a Sylow 2-subgroup of $C_{H}(a) \cap C_{H}(J / O(J))$ and $C_{H}(a) \cap C_{H}(J / O(J))$ is 2-constrained. Clearly $a \in O_{2^{\prime}, 2}\left(C_{H}(a)\right)$ and as $b \nsim a$, we obtain

$$
C_{H}(a) \cap C_{I I}(J / O(J)) / O\left(C_{H}(a)\right) \cong P \text { or } S_{4}
$$

with $b \notin O_{2^{\prime}, 2}\left(C_{H}(a)\right)$ in the latter case. Thus

$$
C_{Y}(J / O(J)) / Y \cap O\left(C_{H}(a)\right) \cong E_{4} \text { or } S_{3} .
$$

Again $a \nsim b$ precludes the possibility that a 3 -element acts on the $E_{4}$. Again we achieve $Y / O(Y)$ a 2-group.

$$
C_{4}=(\tau b)^{H} \cap Z(Q) \text {. }
$$

Proof. As $N_{G}(F)$ stabilizes the series $1 \subseteq\langle a b\rangle \subseteq\langle a, z\rangle \subseteq\langle a, z, \tau b\rangle$, $N_{G}(F)$ acts on $Z(F)$ as a 2-group. Two elements of the coset $\langle a, z\rangle \tau b$ are $H$-conjugate and two are not. The only further fusion possible is $\tau b a \sim \tau b z$.

$$
P \cong D \text { and } P \cong F .
$$

Proof. We have $F=D \times(P \cap F) \times\langle\sigma\rangle$ and as $z$ is fused to $a$ in $N_{G}(F)$, we have $D \cong P \cap F$. It suffices to show $|P| \leqq|D|$. Assume the contrary. As $P^{v}$ acts faithfully on $J / O(J)$ and $P$ has maximal class, $|P|=2|D|$. Further $\left|P: C_{P}(\sigma)\right| \leqq 2$; so $[P, \sigma] \neq 1$ implies $\sigma \sim$ $\sigma a$, i.e., $\tau b \sim \tau b a$, not the case. Thus $P$ and $\left\langle P, P^{v}\right\rangle$ centralize $Z(F)=$ $\langle a, z, \sigma\rangle$. As $\sigma$ acts as a field automorphism on $J / O(J),\left\langle P, P^{v}\right\rangle$ acts as inner or field automorphisms, but not as diagonal automorphisms. Thus $\left\langle P, P^{v}\right\rangle=P \times P^{v} \cong D \times\langle\sigma\rangle \times P$ forces $|P| \leqq|D|$.

(6) We may assume that $b^{H} \cap Z(Q)=C_{3} \cup C_{5}$.

Proof. Suppose not. Then $\tau b a$ is 2-central in $N_{H}(F)$, so $b$ is 2central in $H$. We know that $a$ acts as an outer automorphism on $K$ and that the $K$-classes of involutions in $K\langle a\rangle$ are represented by $a$, $a z \tau, z, z \tau$. Thus every involution in $K\langle a\rangle$ is $H$-fused into $C_{1} \cup C_{2}$. In particular, $b \nsim K\langle a\rangle$. If a 2-element $\eta$ acts on $K$ as a field automorphism, then $m(F)=m(H)=5$ implies $\Omega_{1}(\langle\eta\rangle)=\langle b\rangle$, else $m\left(C_{H}(b)\right)=6$. Pick $\eta$ with $|\langle\eta\rangle|$ as large as possible. If no 2-element acts as a field automorphism, set $\eta=b$. We see that $K\langle a\rangle\langle\eta\rangle$ contains a Sylow 
2-subgroup of $C_{H}(b)$ and of $H$. As $a \sim z \in K \cong O^{2}(H)$ and $b \nsim K\langle a\rangle$, transfer gives $\langle K, a, \eta\rangle \cap O^{2}(H)=\langle K, a\rangle$. As $K\langle a\rangle$ has sectional 2rank 4, we have by Lemma 5.10 and Proposition 2.11,

$$
F^{*}(H) \cong L_{4}(q), \quad U_{4}(q) \text { or } A_{10},
$$

and we are done in this case.

(7) There exists $y \in U$ with $(\tau b z)^{y}=\tau b a$, with $C_{Z(F)}(y)=\langle a, z\rangle$ and with $y$ acting nontrivially on both $D /[D, D]$ and $P /[P, P] . P-$ $\langle a\rangle=P \cap b^{H}$.

Proof. As $\langle\tau b z \tau, b a\rangle \leqq Z(F)$ and $U$ covers $N_{H}(Z(F)) / C_{H}(Z(F))$, there is an element $y \in U$ such that $(\tau b z)^{y}=\tau b a$. At this point the action of $N_{H}(Z(F))$ on $Z(F)$ is completely determined. In particular $U / C_{U}(Z(F)) \cong E_{4}$ and we can choose $y$ so that either $C_{Z(F)}(y)=\langle a, z\rangle$ or so that $C_{Z\left(F^{\prime}\right)}(y)=\langle a \tau, a z\rangle$. We choose it so that $C_{Z\left(F^{\prime}\right)}(y)=\langle a, z\rangle$.

Now $y$ acts on $J$ and normalizes $D$. Further since $\tau b z$ acts as a field automorphism on $J / O(J)$ and $(\tau b z)^{y}=\tau b a$, the structure of Aut $(J / O(J))$ forces $y$ to act nontrivially on $D /[D, D]$.

Cleary $y$ normalizes $P$. As $a \tau b \in Z(F), N_{H}(F)$ contains a Sylow 2-subgroup of $C_{H}(a \tau b)$. Since $N_{H}(Z(F))$ acts as a 2-group on $Z(F)$, any Sylow 2-subgroup of $N_{H}(Z(F))$ contains a Sylow 2-subgroup of $C_{H}(a \tau b)$. In particular $R=C_{U}(a \tau b) \in \operatorname{Syl}_{2}\left(C_{H}(a \tau b)\right)$. As we have seen, we can choose $v \in R$ such that $a^{v}=z$. Note that $U=S R$ with $S \cap$ $R=C_{U}(Z(F))$ and $|U: S|=|U: R|=2$. Now $D^{v} \in \operatorname{Syl}_{2}\left(J^{v}\right)$, so the reasoning in the previous paragraph shows that $y$ acts nontrivially on $D^{v} /\left[D^{v}, D^{v}\right]$. Let $E=D\langle a z, a \tau b\rangle$. We have $D^{v} E=F$ and $D^{v} \cap$ $E=\langle a\rangle$. Since $y$ normalizes $D^{v}, y$ acts nontrivially on the commutator quotient of $F / E$. But also $P E=F$ and $P \cap E=\langle a\rangle=[P, P]$, so our claim is valid.

$$
K / O(K) \cong \operatorname{PSp}(4, q) \quad \text { for some odd } q \geqq 3 .
$$

Proof. Suppose $K / O(K) \cong A_{8}$. Then $L / O(L) \cong A_{8}$ where $L=$ $L\left(C_{H}(a \tau b)\right)$. Now $R \cap L\langle a \tau b\rangle$ contains $A \cong E_{32}$ with

$$
N_{L}(A) / C_{L}(A) \cong S_{3} 2 Z_{2} .
$$

Further $N_{L}(A)$ fuses every involution in $C_{A \cap L}\left(C_{J}(a \tau b) / O\left(C_{J}(a \tau b)\right)\right)$ to $a$. Thus every involution in $C_{A}(J / O(J))-\langle a \tau b\rangle$ is fused to $a$ or $\tau b$. But $C_{A}(J / O(J))=A \cap P \cong E_{4}$, and so must contain two involutions fused to $b$, contrary to $a \nsim b \nsim \tau b$.

(9) $L\left(C_{H}(\langle a z, a\rangle)\right)=1$. If $q=3, C_{H}(\langle a z, a\rangle)=C_{X}(\alpha z) S$, where $X=$ $O\left(C_{H}(a)\right)$. 
Proof. As $P \in \operatorname{Syl}_{2}\left(C_{H}(J / O(J))\right)$ and $P$ is dihedral with Inv $P=$ $\left(P \cap b^{G}\right) \cup\{a\}, C_{H}(J / O(J))$ has a normal 2-complement. Thus by the structure of Aut $L_{2}\left(q^{2}\right)$,

$$
O^{2^{\prime}}\left(C_{H}(a) / O\left(C_{H}(a)\right)\right)=S J O\left(C_{H}(a)\right) / O\left(C_{H}(a)\right) .
$$

Moreover if $q=3, C_{H}(a) / O\left(C_{H}(a)\right)=S J O\left(C_{H}(a)\right) / O\left(C_{H}(a)\right)$. As $C_{J / O(J)}(a z)$ is dihedral and is of order 8 if $q=3$, the claims follow. $a \tau b\rangle))$.

Notation. Set $F=C_{H}(a z), \bar{F}=F /\langle O(F)\langle a z\rangle\rangle, \quad L=L\left(C_{H}(\langle a z\right.$,

$$
q=3
$$

Proof. Suppose that $q>3$. As $C_{F}(a \tau b)$ covers a subgroup of index 2 of $C_{\bar{F}} \overline{(a \tau b)}, \bar{L}$ is subnormal in $C_{\bar{F}} \overline{(a \tau b)}$ and we conclude that $L\left(C_{\bar{F}} \overline{(a \tau b))}=\bar{L} \cong L_{2}(q) \times L_{2}(q)\right.$. Suppose that $\bar{L}$ is not subnormal in $\bar{F}$. Let $\bar{J}_{0}$ be a summand of $\bar{L}$ which is not subnormal in $\bar{F}$. As $\bar{a}$ normalizes $\bar{J}_{0}, \bar{a}$ normalizes a Sylow 2-subgroup, $\bar{P}_{1}$, of $C_{\bar{F}}\left(\bar{J}_{0}\right)$. Let $\bar{E}$ be a maximal $\langle\bar{a}\rangle$-invariant subgroup of $\bar{P}_{1}$ satisfying:

$$
\bar{J}_{0} \text { is not subnormal in } N_{\bar{F}}(\bar{E}) \text {. }
$$

As $\bar{F}$ satisfies the $B(G)$-Conjecture, it follows easily that $\bar{J}_{0}$ projects to a standard component of $N_{\bar{F}}(\bar{E}) / \bar{E}$. Thus $\bar{K}_{0}=\left\langle\bar{J}_{0} L\left(N_{\bar{F}}(\bar{E})\right)\right\rangle$ is a central product of restricted quasi-simple groups. Let $\bar{e}$ be an involution of order 4 in $F$, $\overline{a \tau b} \nsim \overline{a \tau b e}$. By Proposition $2.3(4)$, the possibilities for $\bar{K}_{0}$ are:

$$
\begin{aligned}
& \mathrm{SL}(2, q) * \mathrm{SL}(2, q), L_{2}\left(q^{2}\right), A_{7}, A_{8}, A_{9}, A_{10}, \\
& L_{3}^{\wedge}(4), \operatorname{PSp}(4, \sqrt{q}), L_{4}^{\wedge}(\sqrt{q}), U_{4}^{\wedge}(\sqrt{q}), \Omega^{-}(8, \sqrt[4]{q}) .
\end{aligned}
$$

Now $\bar{a}$ normalizes $\bar{E}$, hence $\bar{K}_{0}$. As $L\left(C_{\bar{K}_{0}}(\bar{a})\right)=1$, a quasisimple component of $\bar{K}_{0}$ must be isomorphic to $\mathrm{SL}(2, q) * \mathrm{SL}(2, q), L_{2}\left(q^{2}\right), A_{7}$, $A_{8}, A_{9}, A_{10}, L_{3}(4), \operatorname{PSp}(4,3), L_{4}(3)$ or $U_{4}(3)$ by Proposition 2.12. As $a z \in Z\left(K_{0}\right), \bar{K}_{0} \not \mathrm{A}_{n}$ for $n \geqq 8$ by Theorem 1.1 of [22].

If $\bar{K}_{0} \cong L_{3}(4)$, then as $e$ has order 4 in $F, K_{0} / O\left(K_{0}\right)$ is a 16-fold covering group of $L_{3}(4)$. As $\bar{F}$ satisfies the $B(G)$-Conjecture, this is impossible by [22]. Suppose that $K_{0} / O\left(K_{0}\right)$ has a component isomorphic to $\mathrm{SL}(2, q), \mathrm{SL}\left(2, q^{2}\right)$ or $A_{7}$. If this component is subnormal in $F / O(F)$, then it is intrinsic and $H$ is known by the main theorem of [4]. This we may repeat our earlier argument to produce a component $K_{1}$ with $\bar{K}_{1}$ not isomorphic to $\mathrm{SL}(2, q), L_{2}\left(q^{2}\right)$ or $A_{7}$. But then we must have $\bar{K}_{1} \cong \operatorname{PSp}(4,3), L_{4}(3)$ or $U_{4}(3)$. Then necessarily $q=9$ and $\bar{J} \cong L_{2}(81)$. It follows that $C_{H}(\langle a, a z\rangle)$ is 2-nilpotent, but the fixed point subgroup of every involutory automorphism of $\operatorname{PSp}(4,3)$, 
$L_{4}(3)$ or $U_{4}(3)$ involves $A_{4}$ by Proposition 2.12, a contradiction.

Thus $\bar{L}$ is subnormal in $\bar{F}$ and, again, $H$ is known by the main theorem of [4].

$$
q \neq 3
$$

Proof. Let $R=C_{U}(a \tau b)$. Since $q=3, K\langle a\rangle\langle b\rangle$ contains a Sylow 2-subgroup of $C_{H}(b)$. Thus $R \subseteq L\langle r\rangle\langle a \tau b\rangle$ for some $r \in \operatorname{Inv}(R)$ with $r \sim a$. We see that $R=\Omega_{1}(R)$ and we can pick $v \in \operatorname{Inv}((R \cap L)-F)$ with $F \cap L\langle r\rangle=F_{1} \times\left(F_{1}\right)^{v}, F_{1} \cong D$. The coset $v F$ contains $2|D|$ involutions and $\left|R: C_{R}(v)\right|=|D|$. If $C_{R}(v)=C_{U}(v)$, then $\left|U: C_{U}(v)\right|=$ $2|D|$ and all involutions in $v F$ are conjugate. By inspection $v \sim_{L} a$ or $a z$ and, correspondingly $v a \tau b \sim_{L} \tau b$ or $\tau b z$. As $a \not \tau b$ and $a z \chi$ $\tau b z$, we conclude that $v \nsim v a \tau b$. Thus $C_{R}(v) \subset C_{U}(v)$. It follows that $U=F C_{U}(v)$ and $S=F C_{S}(v)$. As $F=P \times P^{v} \times\langle a \tau b\rangle$, we have $S=$ $P C_{S}(v), P \cap C_{S}(v)=1$.

Recall that there exists $y \in U$ with $\langle a, z\rangle \subseteq C_{U}(y)$ and $(a \tau b)^{y}=z \tau b$. As $q=3$, we must have $S / P$ isomorphic to a full Sylow 2-subgroup of Aut $(J / O(J))$. It is now clear that we can choose $y$ so that $y \in$ $\operatorname{Inv}\left(C_{S}(v)\right)$ and $y$ acts as a diagonal automorphism on $J / O(J)$.

Now $U=P C_{V}(v)$ and as $v \notin \Phi(U), C_{U}(v)=R_{1} \times\langle v\rangle$ with $R_{1}$ isomorphic to a Sylow 2-subgroup of Aut $L_{2}(9)$. Let $P_{1}=C_{P P} v(v)$. Then $P_{1} \cong D_{8}$ and $P_{1} \leqq C_{V}(v)$. As

$$
C_{L^{\prime}}(v) / P_{1}=\left\langle P_{1} a \tau b, P_{1} y, P_{1} v\right\rangle
$$

with $[a \tau b, y] \in P_{1}$ and $v \notin C_{V}(v)^{\prime}$, we have $C_{U}(v) / P_{1} \cong E_{8} \cong U / P P^{v}$. In particular, $a \tau b \notin \Phi(U)$. We have seen that $N_{I I}(F)=O\left(N_{H}(F)\right) U$ and $F$ is weakly closed in $U$. Thus as $a \tau b \in Z(F)$ and $a \tau b \notin N_{H}(F)^{\prime} N_{H}(F)^{2}$, we have $a \tau b \notin H^{\prime}$ by Lemma 3.10. As $a \tau \sim z \in H, b \notin H^{\prime}$. Thus $P \cap H^{\prime} \cong Z_{4}$ and $J$ is maximal in $H$. Thus $H$ is of restricted type and our conclusion follows by inspection of the possibilities.

As (9) and (10) exhaust all cases, we are done.

\section{LEMMA 8.2. $|P|=4$.}

Proof. Suppose that $|P| \geqq 8$. By Lemma 8.1, $|B| \geqq 4$ and, as $B \cap B^{x}=1,|P| \geqq 16$. Further, $\left|N_{P}(B): B\right|=2$, so $B$ is not normal in $P$.

We let $x_{0}, B_{0}$ be as in $\S 6$. As $A \nsupseteq P$, Proposition 8.2 holds for $B, x_{0}$ and $B_{0}$. Let $L=L\left(C_{G}\left(B_{0}\right) \leqq G_{0}=N_{G}\left(B_{0}\right)\right.$. As $\bar{a} \in Z\left(\left\langle\bar{x}_{0}, \bar{B}\right\rangle\right), \bar{a}$ acts as an inner automorphism on $\bar{L}$. Thus, by Lemma 8.1, we hav $\epsilon$

$$
\begin{aligned}
& L / O(L) \cong L_{4}(q) \quad q \equiv 3(\bmod 4), \quad \text { or } \\
& U_{4}(q) \quad q \equiv 1(\bmod 4) \text {, or } \\
& A_{10} \text {. }
\end{aligned}
$$


By Proposition 6.3(3),

$$
N_{S}\left(B_{0}\right) \in \operatorname{Syl}_{2}\left(N_{G}\left(B_{0}\right) \cap C_{G}\left(\langle a\rangle\left[B, x_{0}\right]\right)\right),
$$

where $\left[B, x_{0}\right] \subseteq\langle a, b\rangle$. It follows from the structure of $L$ as described in Proposition 2.9 that there exists an element $t$ with the following properties:

(a) $t \in N_{L}\left(S_{0}\right)-S_{0}$ and $t^{2} \in S_{0}$.

(b) $D D^{t}=D \times D^{t}=L \cap S_{0}$.

(c) $D^{t}$ acts on $J / O(J)$ as a field automorphism.

(d) $D^{t} \cap P=C_{D^{t}}(J / O(J))$ is cyclic of index 2 in $D^{t}$.

(e) $\left(D^{t} \cap P\right) B / B_{0}$ is dihedral and $a \in\left(\Omega_{1}\left(D^{t} \cap P\right)\right) B_{0}-B_{0}$.

We assume for the next few steps that $N_{G}(S) \subseteq C_{G}(a)$.

(1) $x_{0}$ acts nontrivially on $D^{t}$.

Proof. Clearly $x_{0} \in P_{0}=S_{0} \cap P$, so $x_{0}$ acts on $D^{t} \geqq S_{0}$. Further $P_{0} / B_{0}$ has maximal class by Lemma 5.12 and $\left\langle x_{0}, B\right\rangle / B_{0} \cong D_{8}$. As $D^{t} \cap P$ is cyclic of order at least 4 and projects isomorphically onto $P_{0} / B_{0}$, either $x_{0}$ inverts $D^{t} \cap P$ or $x_{0} \in\left(D^{t} \cap P\right) B_{0}$. In the latter case $x_{0} \notin\left\langle a, B_{0}\right\rangle$ implies that $x_{0}$ acts nontrivially on $D^{t}$.

(2) Let $\left\langle a_{0}\right\rangle=Z\left(D^{t}\right)$. Then $b \in B_{0} \cap Z_{2}(S)$ and $a_{0}=a b$.

Proof. Now $\left\langle a_{0}\right\rangle \subseteq\left[D^{t}, x_{0}\right] \subseteq\left[S, x_{0}\right] \cap P$. If $x_{0} \in Z_{2}(S)$, then as $\langle a\rangle=Z(S) \cap P, a_{0}=a$, whence $a$ is fused to $z$ in $N_{G}(S)$, contrary to $N_{G}(S) \subseteq C_{G}(a)$. Thus $x_{0} \in Z_{3}(S)$ and $a_{0} \in\left[S, x_{0}\right] \cap P=\langle a, b\rangle$. As $b \in B_{0}, a_{0}=a b$.

(3) Let $A=\langle a, z, b\rangle, N=N_{G}\langle A\rangle, C=C_{N}(a)$. Then $N / C \cong S_{3}$ and $z \sim a z \sim b, a \sim b z \sim b a z$ in $N$; $a z \in Z(N)$.

Proof. Let $U=C_{S}\left(a_{0}\right)$. Clearly $|S: U|=2$. Now $t$ interchanges $z$ and $a b$ and fixes $b$. Since $\langle a, z\rangle \subseteq Z(S), A \subseteq Z(U)$ and $U=C_{S}(A)$. Picking $y \in S-U$, we have $a^{y}=a, z^{y}=z$ and as $\langle a, b\rangle \leqq S, b^{y}=a b$. Thus $\langle y, t\rangle$ acts on $A$ as $S_{3}$. Since $N_{G}(S) \subseteq C_{G}(a), N_{G}(S)$ centralizes $\langle a, z\rangle$. So $a \nsim z \nsim a z$ in $G$. The conclusion follows.

We may copy the argument in the proof of Lemma 7.1 to conclude that $C$ acts on $J$ and centralizes $z$. Thus $C / C_{C}(J / O(J))$ has a normal 2-complement.

$$
C=U O(C)
$$

Proof. Let $X$ be the largest subgroup of $C_{C}(J / O(J))$ which is normal in $N$. Clearly $C / X$ has a normal 2-complement. We claim $|X|$ is odd. If not, then $1 \neq X \cap U \leqq U$ implies $E=X \cap \Omega_{1}(Z(U)) \neq 1$. 
As $X$ centralizes $J / O(J), X \cap U \subseteq P$ so that $E \subseteq P . \quad S$ acts as an involution on $E$ and $C_{E}(S) \subseteq Z(S) \cap P$ implies $C_{E}(S)=\langle a\rangle$. Thus $|E| \leqq$ 4. But $X \leqq N$ implies $E \leqq N$ whence $|E| \geqq\left|\left\langle a^{N}\right\rangle\right|=8$, a contradiction.

(5) Let $N_{1}=N_{G}(U), E=\left\langle D^{N_{1}}\right\rangle$. Then $E=D \times D^{t} \times D^{t y}$. Every involution in $D D^{t}$ is conjugate to $z$ or $a$.

Proof. As $N_{1}$ covers $N / O(C), N_{1} / C_{N_{1}}(A) \cong S_{3}$. As $D \leqq S, D$ has $3 N_{1}$-conjugates with centers $\langle z\rangle,\langle a b\rangle,\langle b\rangle$ and as each conjugate is normal in $U, E$ is a direct sum of the conjugates of $D$. Since $t \in N$, one of the $N_{1}$-conjugates of $D$ projects onto $D^{t} O(C) / O(C)$. But $D^{t} \subseteq$ $S_{0} \subseteq U$ and $U$ projects isomorphically onto $U O(C) / O(C)$, so $D^{t}$ is one of the $N_{1}$-conjugates of $D$. Since $y$ normalizes $U$, we have $E=D \times$ $D^{t} \times D^{t y}$. Since $D D^{t} \subseteq L, \operatorname{Inv}\left(D D^{t}\right) \subseteq z^{L} \cup(a b z)^{L}$. As $a b z \sim a$ in $N$, our claim is valid.

$$
a z^{G} \cap D D^{t} \neq \varnothing
$$

Proof. Pick $v \in N_{1}$ so that $\langle v\rangle$ covers $O\left(N_{1} / C_{N_{1}}(A)\right) \cong \boldsymbol{Z}_{3}$ and so that $|v|$ is a power of 3 . From the structure of $N, v^{3} \in N_{1} \cap O(C)$ forces $v^{3} \in C_{N}(U)$. Since $D^{t}$ acts on $J / O(J)$ as a field automorphism of order 2 , we can pick $E_{0}=\left\langle z, e_{1}\right\rangle \subseteq D$ so that

$$
\left|E_{0} \cap L\left(C_{J}\left(D^{t}\right)\right)\right|=2 \text {. }
$$

Letting $\left\{e_{1}, e_{2}, e_{3}\right\}$ be the $\langle v\rangle$-conjugates of $e_{1}$, we see that $\langle v\rangle$ normalizes $F=\left\langle z, e_{1}, a b, e_{2}, b, e_{3}\right\rangle \cong E_{64}$, and we may assume that with respect to the basis $\left\{z, e_{1}, a b, e_{2}, b, e_{3}\right\}, v$ is represented by

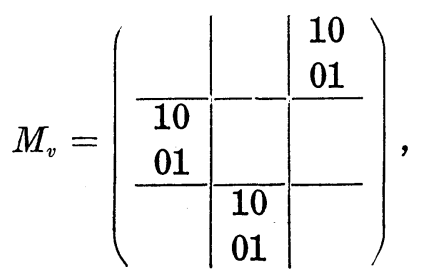

where omitted entries are zero. $F$ acts on $J$ as a group of order 8 and $N_{J}(F)$ acts as $S_{3}$ on $F$. We can pick $r \in N_{D}(F)-F$ so that $r$ acts as

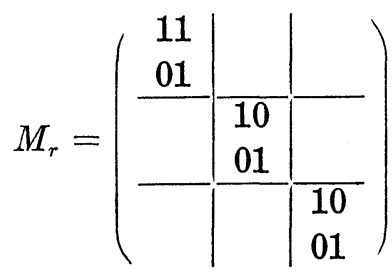


and we can pick $w \in F$ so that $|w|$ is a power of 3 and $\langle r, w\rangle$ acts on $E_{0}$ as $S_{3}$. Clearly $w$ centralizes $F \cap P \supseteqq\langle a b, b\rangle$ and by our choice of $E_{0},\left[w, e_{2}\right] \neq 1$. From the structure of Aut $L_{2}\left(q^{2}\right), w$ must centralize $e_{2} z$. Likewise $w$ centralizes $e_{3}$ or $e_{3} z$. We have $F=E_{0} \times C_{F}(w)$ with $C_{F}(w)$ equal to $\left\langle a b, b, e_{2} z, e_{3}\right\rangle$ or $\left\langle a b, b, e_{2} z, e_{3} z\right\rangle$. In either case the action of $w$ on $E_{0}$ determines its action on $F$, and we may assume

$$
M_{w}=\left(\begin{array}{c|c|c}
01 & 01 & 0 \varepsilon \\
11 & 01 & 0 \varepsilon \\
\hline & 10 & \\
& 01 & \\
\hline & & 10 \\
& & 01
\end{array}\right)
$$

where $\varepsilon=0$ or 1 . In any event

$$
M_{w}\left(M_{v} M_{w}\right)^{2}(a z)=e_{2} z \in D D^{t} .
$$

But now (5) and (6) yield a contradiction, as $z \nsim a z \nsim a$. Thus $N_{G}(S) \nsubseteq C_{G}(a) . \quad N_{G}(S)$ acts on $\langle a, z\rangle=[S, S] \cap Z(S)$. If $a^{u}=a z$ for $u \in N_{G}(S)$, then $P^{u} \cap P=1=P^{u} \cap D$ implies $\left[P^{u}, D\right]=1$, whence $P^{u}$ acts faithfully on $J / O(J)$ as a fours group, contrary to $|P|>8$. Thus we have the following.

(7) Let $U \in \operatorname{Syl}_{2}(G)$ with $S \subset U$. Then $\left|N_{U}(S): U\right|=2$ and if $u \in$ $N_{U}(S)-S$, then $a^{u}=z$ and $P^{u}$ acts faithfully on $J / O(J)$.

$$
B_{0}=\langle b\rangle \text { with } b^{2}=1 \text {. }
$$

Proof. Recall the existence of $t \in N_{L}\left(N_{S}\left(B_{0}\right)\right)$ with $D \times D^{t}=$ $N_{S}\left(B_{0}\right) \cap L$ and $D^{t} \cap P$ cyclic of index 2 in $D^{t}$. $\left[\left(D^{t} \cap P\right) \times B_{0}\right]^{u}$ acts faithfully on $J / O(J)$. The claim is immediate from the structure of $\operatorname{Aut}\left(L_{2}\left(q^{2}\right)\right)$.

(9) $b^{u}$ acts on $J / O(J)$ as a field automorphism and $b \underset{S}{\widetilde{S}} b a$.

Proof. The first assertion is clear. As $b \notin Z(S) \cap P, b^{u} \notin Z(S) \cap P^{u}$, whence some element, $y$, of $S$ acts as a diagonal outer automorphism on $J / O(J)$. Considering the action of $y$ on $P^{u}$ we have

$$
b^{u} \widetilde{S}^{u} z
$$

and conjugating $y$ by $u$ gives

$$
b \underset{S}{\sim} b a .
$$

$$
Z\left(J_{e}(S)\right)=\left\langle z, b^{u}, a, b\right\rangle \text { and }|U: S|=2 \text {. }
$$


Proof. Since $P^{u}$ is faithful in $J / O(J), m\left(P^{u}\right)=m(P) \leqq 3 . \quad\left[D, D^{u}\right]=$ 1 implies $D^{u}$ must act on $J / O(J)$ as a subgroup of $\left\langle z, b^{u}\right\rangle$. As $\left\langle z, b^{u}\right\rangle^{u}=$ $\langle a, b\rangle \subseteq P,\left\langle z, b^{u}\right\rangle \subseteq P^{u}$, whence

$$
D^{u} \cong\left\langle z, b^{u}\right\rangle P \subseteq P P^{u} .
$$

Now $P \cap Z(S)=\langle a\rangle$ and $P^{u} \cap Z(S)=\langle z\rangle$, so $P \cap P^{u}=1$ and $P P^{u}=$ $P \times P^{u}$. Further, $D\left\langle b^{u}\right\rangle \cap P=1$, as $D\left\langle b^{u}\right\rangle$ acts faithfully on $J / O(J)$. Thus $P^{u}$ contains a subgroup isomorphic to $D\left\langle b^{u}\right\rangle$, whence $m\left(P^{u}\right)=$ $3=m(P)$ and $m(S)=6$. Clearly $J_{e}(S)=J_{e}(P) \times J_{e}\left(P^{u}\right) \cong D \times\left\langle b^{u}\right\rangle \times$ $D \times\left\langle b^{u}\right\rangle$. In particular $Z\left(J_{e}(S)\right) \cong E_{16}$. Also exactly as in the proof of Lemma 7.1, $|U: S|=2$.

Set $F=J_{e}(S), A=Z(F)$. Fusion in $A$ is controlled by $N_{G}(F)$ and $\langle a, z\rangle=[F, F] \cap A$ is invariant under $N_{G}(F)$. Thus

$$
\begin{aligned}
(a z)^{G} \cap A & =\{a z\} \\
a^{G} \cap A & =\{a, z\} .
\end{aligned}
$$

The following fusion occurs in $U$ :

$$
\begin{gathered}
a b \sim b \sim b^{u} \sim b^{u} z, \quad z b \sim a z b \sim a z b^{u} \sim a b^{u} . \\
a \sim z \text { in } N_{G}(F) \cap C_{G}(b) . \quad \text { Thus } a b \sim b z .
\end{gathered}
$$

Proof. $C_{G}(b)=G_{0}$ and we know that $F^{*}\left(\bar{G}_{0} / O\left(\bar{G}_{0}\right)\right)$ is simple, where $\bar{G}_{0}=G_{0} /\langle b\rangle$. As $F \subseteq G_{0}, \bar{a} \in[\bar{F}, \bar{F}] \subseteq \bar{L}$. Thus $a \in L$ or $a b \in L$. In the latter case $a \sim b$, contrary to $a^{G} \cap A=\{a, z\}$. Thus $a \in L$ and $a \sim_{L} z$. Thus $a \sim z$ in $N_{G_{0}}(F)$.

Now $8 \leqq\left|b^{a} \cap A\right| \leqq 12 . \quad C_{A}(u)=\left\langle a z, b b^{u}\right\rangle$ and, as $b b^{u}$ acts on $J / O(J)$ as a field automorphism, $b b^{u} \notin Z(S)$. Thus $C_{A}(U)=\langle a z\rangle$. Consequently any involution in $A$ which is 2-central in $G$ is fused to $a z$, and so $b$ is not 2-central in $G$. Since $\langle b, a\rangle=A \cap P \unlhd S$ and $|U: S|=2$, $\left|U: C_{U}(b)\right| \leqq 2\left|S: C_{S}(b)\right|=4$. Thus $\left|b^{G} \cap A\right|=10$ or 12 . Clearly no 5-element acts on $F$, so $\left|b^{a} \cap A\right|=12$ and

$$
b^{G} \cap A=A-\langle a, z\rangle .
$$

Consequently there exists $\lambda \in N_{G}(F)$ of order $3^{a}$ acting nontrivially on $A$. As $\lambda$ centralizes $\{a, b\}, \lambda$ must normalize $P \cap A=\langle a, b\rangle$, whence $\lambda$ centralizes $\langle a, z, b\rangle$, a contradiction.

This completes the proof of Lemma 8.2.

LeMma 8.3. $|P| \neq 4$.

Proof. Assume that $|P|=4$ and let $P=\langle a, b\rangle$. It is clear that $x$, the unbalancing involution, acts nontrivially on $P$ with $[b, x]=a$. Thus $x$ acts as an outer diagonal automorphism on $J / O(J)$. Since $Q=$ 
$N_{S}(B) \in \operatorname{Syl}_{2}\left(C_{G}(a) \cap N_{G}(B)\right)$, we know from the structure of PSp $(4, q)$ and $A_{8}$ described in Proposition 2.6 that there exists $g \in N_{K}(Q)-Q$ such that $g^{2} \in Q$ and $a^{g}=a \tau$ where $\tau$ is a non-2-central involution of $K$ acting on $J / O(J)$ as a field automorphism and $\tau z$ is 2-central in $K$. In particular, $\Omega_{1}(S)=D P\langle x, \tau\rangle$.

$$
b \in F^{*}(G) \text {. }
$$

Proof. By the minimality of $G, G=\left\langle F^{*}(G), x\right\rangle$ with $F^{*}(G)$ simple. Thus if $b \notin F^{*}(G)$, then $F^{*}(G)$ is a balanced group. Then $J$ is simple and $\langle a\rangle=C_{F^{*}(G)}(J)$. Thus $J$ is a standard component in $F^{*}(G)$, whence $G$ satisfies one of the conclusions of Theorem B.

$$
q=3 \text {. }
$$

Proof. Suppose $q \geqq 5$. We have $b^{x g}=(b a)^{g}=b a \tau$. Consider $H=$ $C_{G}(\langle a, b a \tau\rangle)$. Since $|P|=4$ and $a \nsim b, C_{G_{G}(a)}(J / O(J))$ is 2-constrained; so

$$
C_{G_{\left.G^{(}\right)}(J)}(J / O(J))=O\left(C_{G}(a)\right) P .
$$

It follows that $L\left(C_{J}(b a \tau)\right) P$ covers $L(H) O_{2^{\prime}, 2}(H) / H \cap O\left(C_{G}(a)\right)$. In particular

(a) $O(H) \subseteq O\left(C_{G}(a)\right)$, and

(b) $P\left(H \cap O\left(C_{G}(a)\right)=C_{H}(L(H) / O(L(H)))\right.$.

Now look at the action of $a$ on $L=L\left(C_{G}(b a \tau)\right)$. Let $\bar{L}=L / O(L)=$ $\operatorname{PSp}(4, q)$. As $L(H) / O(L(H)) \cong L_{2}(q), q \geqq 5$, we know by $L$-Balance and structure of $\operatorname{Aut}(\operatorname{PSp}(4, q))$ that $C_{\bar{L}}(a)$ contains $\overline{L(H)} \times Z_{(q+\varepsilon) / 2}$ where $\varepsilon= \pm 1$. If $q+\varepsilon$ is not a power of 2 , then $H \cap O\left(C_{G}(a)\right)$ covers the odd part of the $\boldsymbol{Z}_{(q+\varepsilon) / 2}$ whence $(b a \tau, a, L)$ is an unbalancing triple, contrary to the hypothesis of Theorem B. Thus $q+\varepsilon$ is a power of 2 and, as the $\boldsymbol{Z}_{(q+\varepsilon) / 2}$ must be isomorphic to a subgroup of $P$, we have $q+\varepsilon=2$ or 4 , whence $q=3$ or 5 . Further, we know by the action of $a$ on $K$ that there is a conjugate, $e$ of $a$ acting on $L$ as an outer diagonal automorphism on $\bar{L}$. If $a$ acts as an inner automorphism on $\bar{L}$, then $C_{\langle L, e\rangle /(L)}(a)$ contains a cyclic group of order 4 . Since $P$ is elementary, we conclude that $a$ acts as an outer automorphism. In this case $q+\varepsilon=2$ and $q=3$.

(3) $S=D P\langle x, \tau\rangle$ and $Q=D P\langle\tau\rangle \cong D_{8} \times E_{8} . \quad Z(Q)=\langle z, a, b, \tau\rangle$ and we have

(i ) $a \stackrel{g}{\sim} a \tau \stackrel{x}{\sim} a \tau z \stackrel{g}{\sim} a z$

(ii) $b \stackrel{x}{\sim} a b \stackrel{g}{\sim} a b \tau \stackrel{x}{\sim} \tau b z$

(iii) $\quad \tau b \stackrel{x}{\sim} \tau z a b \stackrel{g}{\sim} a b z \stackrel{x}{\sim} b z$ 
(iv) $\tau z \stackrel{x}{\sim} \tau \widetilde{K} z$

Proof. Everything but the fusion is clear. Recall that in $N_{K}(Q)-Q$ we have an element $g$ with $g^{2} \in Q$ and $a^{g}=a \tau$. Further $b^{g}=b, \tau^{g}=\tau$ and $z^{g}=z$. We know $b^{x}=b a, a^{x}=a, z^{x}=z$ and from the structure of Aut $\left(L_{2}\left(q^{2}\right)\right), \tau^{x} \in \tau z P$. Since $|x|=2, \tau^{x} \in\{\tau z, \tau z a\}$. In the latter case $(\tau z)^{x g}=(\tau a)^{g}=a$. But $|S|=2^{7}$, while $K\langle a, b\rangle$ contains a Sylow 2-subgroup of $C_{G}(b)$ of order $2^{8}$ and $\tau z$ is 2-central in $K\langle a, b\rangle$. Thus $a \nsim_{G} \tau z$ and $\tau^{x}=\tau z$.

Likewise $a \chi_{a} b$. Further $\tau z$ lies in the commutator subgroup of a Sylow 2-subgroup of $C_{G}(\langle\tau z, b\rangle)$, while $b \notin\left[C_{G}(b), C_{G}(b)\right]$. Thus $b \chi_{G} \tau z$.

We now see that $\langle x, g\rangle$ acts as a dihedral group of order 8 on $Z(Q)$ with the indicated fusion. Further $z \sim \tau$ in $K$.

(4) No two of $a, b, b z, \tau z$ are fused in $G$.

Proof. From the remarks in (3), it suffices to show that $b z$ is not fused to any of the other involutions. Pick $R \in \operatorname{Syl}_{2}\left(C_{G}(b)\right)$ with $\langle Q, g\rangle \subseteq R . \quad R_{0}=C_{R}(b z) \in \operatorname{Syl}_{2}\left(C_{G}(\langle b, b z\rangle)\right)$ with $R_{0} \cong D_{8} \times D_{8} \times Z_{2}$. If $b z \sim{ }_{G} a$, then $\left|R_{0}\right|=|S|$ implies $R_{0} \in \operatorname{Syl}_{2}\left(C_{G}(b z)\right)$. But $a \in[S, S]$ while $b z \notin\left[R_{0}, R_{0}\right]$, so $a \nsim_{G} b z$.

Suppose $b \sim_{G} b z$ and consider $H=C_{G}(\langle a, b z\rangle)$. As in (2), we see that $H / H \cap O\left(C_{G}(a)\right)$ is a 2-group. On the other hand, a cannot act on $L=L\left(C_{G}(b z)\right)$ in such a way that $C_{L}(a)=O_{2^{\prime}, 2}\left(C_{L}(a)\right)$. Thus $b \varkappa_{G} b z$.

Finally, suppose $b z \sim_{G} z \tau$ and pick $T \in \operatorname{Syl}_{2}\left(C_{G}(b z)\right)$ with $R_{0} \leqq T$. We know $z \tau \sim_{G} z$ and $z \in Z(\langle Q, x, g\rangle)$. Thus $|T| \geqq 2^{9}$. In particular, $R_{0} \subset T$. Let $v \in N_{T}\left(R_{0}\right)$. Since $v$ normalizes $\sigma^{11}\left(R_{0}\right)=\langle z, \tau\rangle$ and $b \sim_{G} b z \sim$ $b \tau$ (and $b \neq b^{v}$ ), we have $b^{v}=b z \tau$. Hence

$$
\left|N_{T}\left(R_{0}\right): R_{0}\right|=\left|N_{T}\left(R_{0}\right): N_{T}\left(R_{0}\right) \cap C_{T}(b)\right|=2
$$

and $R_{0}=J_{e}\left(N_{T}\left(R_{0}\right)\right)$. But now $T=N_{T}\left(R_{0}\right)$, whence $|T| \leqq 2^{8}$, a contradiction.

(5) Let $E_{0}$ be the unique $E_{16}$ in $R \cap K$. Let $E=\left\langle E_{0}, b\right\rangle, N=N_{G}(E)$, $\bar{N}=N / C_{N}(E)$. Then $\bar{N} \cong S_{6}$.

Proof. $\overline{N \cap K\langle a\rangle} \cong S_{5}$ or $S_{3} 2 Z_{2}$ according as $K / O(K) \cong \operatorname{PSp}(4,3)$ or $A_{8}$. Further $|R|=2^{8}$ implies $b$ is not 2-central in $G$, so we may pick $U \in \operatorname{Syl}_{2}(G)$ with $R \subset U$ and $N_{U}(E) \in \operatorname{Syl}_{2}(N)$. Pick $v \in N_{U}(R)-R$ with $v^{2} \in R$. As $Z(R)=\langle z \tau, b\rangle, b^{v}=b z \tau$. The $\overline{N \cap K\langle a\rangle}$-classes of involutions are represented by $z$ and $z \tau$ in $E_{0}$ and $b, b z, b z \tau$ in $E-E_{0}$. We see that $E_{0}$ is strongly closed in $E$ with respect to $G$. Further $b z \varkappa_{G} b z \tau$. Letting $C_{b z}$ denote the $\overline{N \cap K\langle a\rangle}$-class of $b z$, etc. we see 
that $C_{b z}$ and $C_{b z \tau} \cup\{b\}$ are $N$-classes.

Suppose $K / O(K) \cong \operatorname{PSp}(4,3)$. Then $\left|C_{z \tau}\right|=\left|C_{z=b}\right|=5$ and $\left|C_{z}\right|=$ $\left|C_{b z}\right|=10$. Further $\left\langle C_{b z z}, b\right\rangle=E$ so that, considering the action of $\bar{N}$ on $C_{b z \tau} \cup\{b\}$, we see that $\bar{N}$ acts as a subgroup of $S_{6}$. As $\overline{N \cap K\langle a\rangle} \cong S_{5}$ and $\overline{N \cap K\langle a\rangle} \subset \bar{N}$, it follows that $\bar{N} \cong S_{6}$.

Next suppose $K / O(K) \cong A_{8}$. Then $\left|C_{z \tau}\right|=\left|C_{z \tau b}\right|=\left|C_{z \tau b}\right|=9$ and $\left|C_{z}\right|=\left|C_{b z}\right|=6$. Now $\left\langle C_{b z}\right\rangle=E$ and considering the action of $\bar{N}$ on $C_{b z}$ we again obtain $\bar{N} \cong S_{6}$.

(6) Define $C=\left\{e_{1}, e_{2}, e_{3}, e_{4}, e_{5}, e_{6}\right\}$ to be the elements of the $\bar{N}$-class of size 6 in $E-E_{0}$. Let $W=N_{U}(E)$. Then $C_{W}(E)=E$ and if $w \in \operatorname{Inv}(N)$ acts as a transposition on $C$, then $W \sim_{G} a, b$ or $b z$.

Proof. Since $\bar{N}$ acts as $S_{6}$ on $C$, it is clear that as an $S_{6}$-module, $E$ is the quotient of the permutation module by its 1-dimensional fixed subspace. In particular, all involutions of $E_{0}$ are fused in $\bar{N}$.

One shows by direct calculation that $\left|\left[E_{0}, a\right]\right|=2$. Indeed $\left|\left[E_{0}, v\right]\right|=2$ for any $v \in \operatorname{Inv}(R-(R \cap K) E) . \quad$ As $[a, b]=1,|[E, a]|=2$ and $a$ acts on $C$ as a transposition. Let $W=N_{U}(E)$. Since $C_{R}(E)=$ $E$ and $C_{W}(E) \subseteq C_{G}(b)$, we have $E=C_{W}(E)$.

Let $w$ be any involution of $N$ acting as a transposition on $C$. Clearly $w$ is $N$-fused to $a C_{W}(E)=a E$. The $K\langle a\rangle$-classes of $K a$ are represented by $a$ and $a z \tau$. Thus any involution in $a E$ is $K\langle a\rangle$-conjugate to $a, a b, a z \tau, a z \tau b$ or $b$. The claim follows.

$$
W \neq U \text {. }
$$

Proof. Assume $W=U$ and let $N_{0}$ be the inverse image in $G$ of $\bar{N}^{\prime}$. Since $a \in N-N_{0}$ and $a \in[S, S]$, it follows that $a$ has a $G$-conjugate in $N_{0} . \quad \bar{N}_{0}$ contains just one class of involutions and from the structure of $\overline{N \cap K\langle a\rangle}$ it follows that $\overline{N_{0} \cap K}$ contains involutions. Thus $\overline{R \cap K}$ contains involutions, whence $\bar{a}$ is conjugate in $\bar{N}$ to $\bar{e} \epsilon$ $\overline{R \cap K}$. It follows that $a \sim e \in(R \cap K) E$. But every involution in $(R \cap K) E$ is $K\langle a\rangle$-fused to $z, z b, z \tau, z \tau b$, or $b$ none of which is $G$-conjugate to $a$. Thus $W \neq U$.

(8) Let $y \in N_{U}(W)-W$. Then we may assume that
(i ) $\bar{E}^{v}=\langle(12),(34),(56)\rangle$
(ii) $\bar{E}_{0}^{y}=\langle(12)(34),(12)(56)\rangle$
(iii) $E \cap E^{y}=\left\langle e_{1}+e_{2}, e_{3}+e_{4}\right\rangle$
(iv) $E_{0} E_{0}^{y}$ is isomorphic to a Sylow 2-subgroup of $L_{3}(4)$.

Proof. We may assume that $\bar{W}$ acts on $C$ as $\langle(12)(34),(13)(24)$, (12), (56) $\rangle$. Every involution in $E_{0}$ is fused to $z$, whence $\bar{E}_{0}^{y}$ contains no transpositions. Suppose $\left|E \cap E^{y}\right|=16$. Then $e^{y} \in E^{y}-E$ acts on 
$E$ as a transposition and every involution of $E e^{y}$ lies in $E^{y}$. Then we may assume that $e^{y} \sim a$, not the case as $e \sim z, b$ or $b z$. As $\bar{W} \cong$ $D_{8} \times \boldsymbol{Z}_{2}, \bar{E}^{y}$ is elementary of order at most 8 and $\bar{E}^{y} \supseteqq \bar{W}$. One now verifies the claim in a straightforward way.

$$
\left|N_{U}(W): W\right|=2 \text {. }
$$

Proof. Let $u \in N_{U}(W)-(W \cup W y)$. Since $y$ was arbitrary in (8), $\bar{E}^{y}=\bar{E}^{u}$ and $E \cap E^{y}=E \cap E^{u}=E \cap E^{u y^{-1}}$. Thus $\left|E^{u} \cap E^{y}\right|=$ $\left|E \cap E^{u}\right|=4$. Since all involutions of $E_{0} E_{0}^{y}$ lie in $E \cup E^{y}$, this yields $\left|E^{u} \cap E_{0} E_{0}^{y}\right|=4$. We know from the fusion of involutions that $E_{0} \leqq N$. Thus $E_{0} E_{0}^{y}$ is normal of index 4 in $E E^{y}$. Our conditions imply $E E^{y}=E E^{u}$, a contradiction.

(10) $E E^{y}$ is weakly closed in $N_{U}(W)$ with respect to $G$.

Proof. Let $F=E^{h}$ be a $G$-conjugate of $E$ with $F \cong N_{U}(W)$ and $F_{0}=E_{0}^{h}$. Suppose $F \nsubseteq W$. Then $F \cap E E^{y}$ lies in $X$, the inverse image in $E E^{y}$ of $C_{E E^{y / E \cap E^{y}}}(f)$, where $f \in F-W$. Now $\left|X: E \cap E^{y}\right|=8$ and $\left|X: X \cap E_{0} E_{0}^{y}\right|=2$. As all involutions of $X \cap E_{0} E_{0}^{y}$ lie in $E_{0} \cap E_{0}^{y}=$ $E \cap E^{y}$, we conclude $|X \cap F| \leqq 8$. As $\left|N_{U}(W): E E^{y}\right|=2^{4}$ and $|F|=$ $2^{5}$, we must have $|X \cap F|=8$, whence $E \cap E^{y} \subseteq F$. But also $F$ covers $N_{U}(W) / E E^{y}$ and $E E^{y}=C_{W}\left(E \cap E^{y}\right) \subset W$, a contradiction. Thus $F \subseteq W$.

We assume $F \nsubseteq E E^{y}$. Then $\bar{F}$ is elementary and $\bar{F} \varsubsetneqq \overline{F^{y}}$, so $\bar{F}$ contains at least one of

$$
(13)(24) \quad(13)(24)(56) \quad(14)(23) \quad(14)(23)(56) .
$$

The last two possibilities are handled in the same way as the first two, so assume $\bar{F}$ contains (13)(24) or (13)(24)(56). Consequently

$$
C_{E}(F) \subseteq C_{E}(\langle(13)(24)\rangle)=\left\langle e_{1}+e_{3}, e_{2}+e_{4}, e_{5}\right\rangle,
$$

or

$$
C_{E}(F) \subseteq C_{E}(\langle(13)(24)(56)\rangle)=\left\langle e_{1}+e_{3}, e_{2}+e_{4}, e_{1}+e_{2}+e_{5}\right\rangle .
$$

In either case $E \cap F \subseteq C_{E}(F)$ implies

$$
|E \cap F| \leqq\left|C_{E}(F)\right| \leqq 8 \text { and }|\bar{F}| \geqq 4
$$

If $|\bar{F}|=8$, then $\bar{F}=\langle(12)(34),(13)(24),(56)\rangle$ and $\left|C_{E}(\bar{F})\right|=2$, forcing $|F| \leqq 16$, not the case. Thus $E \cap F=C_{E}(\bar{F})$. As $\left\langle e_{1}+e_{3}, e_{2}+e_{4}\right.$, $\left.e_{1}+e_{2}+e_{5}\right\rangle$ is not centralized by a fours group in $\bar{W}$, we must have $C_{E}(\bar{F})=\left\langle e_{1}+e_{3}, e_{2}+e_{4}, e_{5}\right\rangle$ and $\bar{F}=\langle(13),(24)\rangle$.

We know that $E_{0}$ is strongly closed in $E$ whence $E \cap F_{0} \subseteq E_{0}$. Thus 


$$
\left|E \cap F_{0}\right| \leqq\left|E_{0} \cap C_{E}(\bar{F})\right|=4,
$$

whence $\bar{F}_{0}=\bar{F}$. But then some element of $F_{0}$ acts as a transposition on $E$ and so some element of $E_{0}$ is $G$-fused to $a, b, b z$, not the case.

$$
U=N_{U}(W)
$$

Proof. By (10) it suffices to show $N_{U}(W)=N_{U}\left(E E^{y}\right)$. Assume not and pick $w \in\left(N_{U}\left(E E^{y}\right) \cap N_{U}\left(N_{U}(W)\right)\right)-N_{U}(W)$, with $w^{2} \in N_{U}(W)$. $E_{0}^{w}$ contains no element acting on $E$ as a transposition, whence $E_{0}^{w} \subseteq$ $E E_{0}^{y}$. Likewise $E_{0}^{w y} \subseteq E E_{0}^{y}$, whence $E_{0}^{w} \subseteq E^{y^{-1}} E_{0}=E^{y} E_{0}$. Thus $E_{0}^{w} \subseteq$ $E E_{0}^{y} \cap E_{0}^{y} E_{0}=E_{0} E_{0}^{y}$. Replacing $w$ by $w y$ if necessary we may assume $E_{0}^{w}=E_{0}$. As $\bar{W}$ acts faithfully on $E_{0}$, we have

$$
E^{w} \subseteq C_{E E}^{y}\left(E_{0}\right)=E,
$$

contrary to our choice of $w$.

$$
b \notin G^{2} .
$$

Proof. Let $M=N_{G}\left(E E^{y}\right)$. For any $w \in M$ the preceding argument has shown $E_{0}^{w} \subseteq E_{0} E_{0}^{y}$. Since the same argument shows $E_{0}^{y w} \subseteq$ $E_{0} E_{0}^{y}$, we conclude $E_{0} E_{0}^{y} \leqq M$. Let $\bar{M}=M / E_{0} E_{0}^{y}$. Since $E \cap E^{y}=$ $Z\left(E E^{y}\right) \leqq U$ and $W$ acts nontrivially on $E \cap E^{y}$, we have
(a) $U=W U_{0}$
(b) $U_{0}=C_{U}\left(E \cap E^{y}\right)$
(c) $W \cap U_{0}=E E^{y}$.

In particular, any element of $U_{0}-E E^{y}$ permutes $E$ and $E^{y}$, whence $\bar{U}_{0} \cong D_{8}$. Further $E \leqq W, E^{y} \leqq W$ imply that $\bar{W}$ is abelian, and it follows that $\bar{U} \cong D_{8} \times \boldsymbol{Z}_{2}$ or $D_{8} * \boldsymbol{Z}_{4}$. Let $e \in \operatorname{Inv}\left(E-E_{0}\right)$. We have in either case $\bar{e} \notin \bar{U}^{2}$. Also $C_{\bar{M}}\left(\bar{E} \bar{E}^{y}\right) / \bar{E} \bar{E}^{y}$ has a Sylow 2-subgroup of order two, whence $C_{\bar{M}}\left(\bar{E} \bar{E}^{y}\right)$ has a normal 2-complement. Once we show $\bar{M} / C_{\bar{M}}\left(\bar{E} \bar{E}^{y}\right) \cong Z_{2}$, we have $\bar{M}=\bar{U} O(\bar{M})$ and $e \notin M^{2}$. The only other possibility is $\bar{M} / C_{\bar{M}}\left(\bar{E} \bar{E}^{y}\right) \cong S_{3}$, in which case $\lambda \in M$ of order $3^{m}$ with $\lambda$ acting nontrivially on $E E^{y} / E_{0} E_{0}^{y}$. But the structure of $E_{0} E_{0}^{y}$ implies that $\lambda$ normalizes $E_{0}$ and $E_{0}^{y}$, hence normalizes $E=C_{E E^{y}}\left(E_{0}\right)$ and $E^{y}$. But then $\lambda$ acts trivially on $E E^{y} / E_{0} E_{0}^{y}$.

Now suppose $e \in G^{9}$ and apply Lemma 3.10. There exists $V \subset E E^{y}$ such that

(1) $C_{E E}(e) \subseteq V$ and

(2) The transfer $V_{E^{\prime} E^{y} \rightarrow V}(e) \notin \Phi\left(E E^{y}\right)$.

Choosing $e=e_{5} \in E-E_{0}$, we see that $\left|E E^{y}: C_{E E^{y}}\left(e_{5}\right)\right|=2$ and $\Phi\left(C_{E E^{y}}\left(e_{5}\right)\right)=$ $E \cap E^{y}=\Phi\left(E E^{y}\right)$. Thus $V=C_{E E^{y}}\left(e_{5}\right)$ and $\left|E E^{y}: V\right|=2$. Hence $V_{E E^{y \rightarrow V}}\left(e_{5}\right) \in$ 
$\Phi\left(E E^{y}\right)=\Phi(V)$, contrary to Lemma 3.10. Thus $e_{5} \notin G^{2}$.

Now $e_{5}$ is $N_{G}(E)$-fused to $b$ or $b z$. As $z \in G^{2}$, we conclude in either case that $b \notin G^{2}$. But by (1), $b \in F^{*}(G)=G^{2}$, a contradiction.

This completes the proof of Lemma 8.3. As Lemmas 8.2 and 8.3 are mutually contradictory, the proof of Theorem B is complete.

9. The proof of Corollary C. In this section $G$ will be a fixed counterexample to Corollary $\mathrm{C}$ and $(a, x, J)$ will be a fixed unbalancing triple satisfying

(1) $J / O(J) \cong A_{7}$.

(2) $x \in E$, a fours subgroup of $C_{G}(a) \cap N_{G}(J)$ with $\Delta=C_{G}(a) \cap$

$\left(\bigcap_{e \in E^{\sharp}} O\left(C_{G}(e)\right)\right)$ and $[J, \Delta]=J$.

(3) $\left|C_{G}(a) \cap N_{G}(J)\right|_{2}$ is maximal subject to (1) and (2).

Suppose that $(a, x, J)$ is a maximal unbalancing triple in $G$. Then either conclusion (1) or (3) of Theorem B must hold. If (1) holds, then as $G$ is not 2-balanced, $F^{*}(G) \cong A_{n}$ for some odd $n \geqq 11$. If (3) holds, then by Lemma $3.5, J$ is maximal in $G$. Now by Theorem 1.5 and the main theorems of [7], [16] and [18], $F^{*}(G)$ is isomorphic to $A_{9}, A_{11}$ or $H e$. Again, as $G$ is not 2-balanced, $F^{*}(G) \cong A_{11}$.

Thus we may assume that $(a, x, J)$ is not a maximal unbalancing triple in $G$. Thus one of the two conditions in the definition of maximal unbalancing triple must fail to hold. Let $S \in \operatorname{Syl}_{2}\left(C_{G}(a) \cap\right.$ $\left.N_{G}(J)\right)$ and let $P=C_{S}(J / O(J)), D=S \cap J$. Let $b \in \operatorname{Inv} C_{P}(S)$ and let $K$ be a 2-component of $\left\langle J^{L\left(G_{G}(b)\right)}\right\rangle$. The proof of Lemma 3.8 shows that if $\Delta_{1}=C_{\Delta}(b)$, then $\left[K, \Delta_{1}\right]=K=[K, E]$. Thus $N_{G}(K)$ is not 2balanced and, as $G$ is a minimal counterexample to the Unbalanced Group Conjecture, $K / Z^{*}(K) \cong A_{n}$ for some odd $n \geqq 7$. By [25] and [28], $K / O(K) \cong A_{7}$. Thus $(b, x, K)$ has the same properties as $(a, x, J)$ with $\left|C_{G}(b) \cap N_{G}(K)\right|_{2} \geqq|S|$. It follows from the choice of $(a, x, J)$ that $S \in \operatorname{Syl}_{2}\left(C_{G}(b) \cap N_{G}(K)\right)$ for all $b \in \operatorname{Inv} C_{P}(S)$. Thus condition 1 in the definition of a maximal unbalancing triple fails to hold.

Now pick $B \subseteq C_{P}(x)$ to satisfy the conclusions of Lemma 3.6 and let $K$ be a component of $\left\langle J_{B}^{L\left(G_{G}^{(B)}\right)}\right\rangle$. Then $N_{G}(K)$ is unbalanced and as above $K / Z^{*}(K) \nRightarrow A_{n}$ for any $n$. On the other hand $J_{B}$ projects onto a standard component of $K / Z^{*}(K)$. Thus $K / O(K) \cong H e$. As in $\S 5, N_{P}(B)=\langle a\rangle \times B$ and $B \cap B^{e}=\langle 1\rangle$ for some $e \in E$, since Aut $H e$ is 2-balanced. Thus $\langle a\rangle=C_{P}(S)$. Also $a \sim a z$ in $K\langle a\rangle$. Hence $a \sim a z$ in $N_{G}(S)$. Let $n \in N_{G}(S)$ with $a^{n}=a z$. Then $P \cap P^{n}=\langle 1\rangle=D \cap P^{n}$. Thus $P^{n}$ acts faithfully on $J / O(J)$ and centralizes $D$. So $P^{n}$ is isomorphic to $Z_{2} \times Z_{2}$. But $E \subseteq D \times P$ and $E$ does not centralize $B$, a contradiction. 


\section{REFERENCES}

1. M. Aschbacher, Finite groups with a proper 2-generated core, Trans. Amer. Math. Soc., 197 (1974), 87-112.

2. - On finite groups of component type, Illinois J. Math., 19 (1975), 87-115.

3. - Standard components of alternating type centralized by a 4-group, to appear.

4. — A characterization of the Chevalley groups over finite fields of odd order, Annals of Math., (2) 106 (1977), 353-398.

5. N. Burgoyne, Finite groups with Chevalley-type components, Pacific J. Math., 72 (1977), 341-350.

6. N. Burgoyne and C. Williamson, Semisimple classes in Chavalley-type groups, Pacific J. Math., 70 (1977), 83-100.

7. R. Foote, Finite groups with maximal 2-components of type $L_{2}(q), q$ odd, to appear Proc. London Math. Soc., 37 (1978), 422-458.

8. R. Gilman, Components of finite groups, Comm. in Algebra, 4 (1976), 1133-1198.

9. D. Gorenstein, The classification of finite simple groups $I, I I$, to appear as a monograph of the Amer. Math. Soc.

10. D. Gorenstein and K. Harada, On finite groups with Sylow 2-subgroups of type $A_{n}, n=8,9,10,11$, Math. Z., 117 (1970), 207-238.

11. —_, Finite groups with Sylow 2-subgroups of type PSp $(4, q), q$ odd, J. Fac. Sci. Univ. Tokyo, 20 (1973), 341-372.

12. - Finite groups whose 2-subgroups are generated by at most 4 elements, Mem. Amer. Math. Soc., 147 (1974).

13. D. Gorenstein and J. H. Walter, Balance and generation in finite groups, J. Algebra, 33 (1975), 224-287.

14. R. Griess and R. Solomon, Finite groups with unbalancing 2-components of $\left\{L_{3}(4)\right.$, He\}-type, to appear in J. Algebra.

15. K. Harada, On the simple group $F$ of order $2^{14} \cdot 3^{6} \cdot 5^{6} \cdot 7 \cdot 11 \cdot 19$, in "Proceedings to to the Conference on Finite Groups," Academic Press, 1976, 119-276.

16. M. Harris, Finite groups having an involution centralizer with a 2-component of dihedral type II, Illinois J. Math., 21 (1977), 621-647.

17. - $\mathrm{PSL}(2, \mathrm{p})$-type 2-components and the unbalanced group conjecture, to appear.

18. M. Harris and R. Solomon, Finite groups having an involution centralizer with a 2-component of dihedral type I, Illinois J. Math., 21 (1977), 575-620.

19. J. H. Lindsey, II, Finite linear groups of degree six, Canad. J. Math., 23 (971), 771-790.

20. D. Mason, Finite simple groups with Sylow 2-subgroup dihedral wreath $Z_{2}$, J. Algebra, 26 (1973), 10-68.

21. - Finite simple groups with Sylow 2-subgroups of type $\operatorname{PSL}(4, q), q$ odd, J. Algebra, 26 (1973), 75-97.

22. C. K. Nah, Uber endliche einfach Gruppen die eine standard Untergruppe $A$ besitzen derart, das $A / Z(A)$ zu $L_{3}(4)$ isomorph ist, Ph.D. Dissertation, Johannes Gutenberg Universitat, Mainz, 1975.

23. G. Seitz, Standard subgroups of type $L_{n}\left(2^{a}\right)$, J. Algebra, 48 (1977), 417-438.

24. R. Solomon, Finite groups with Sylow 2-subgroups of type. 3, J. Algebra, 28 (1974). 182-198.

25. R. Solomon, Finite groups with intrinsic 2-components of type $\hat{A}_{n}$, J. Algebra, 33 (1975), 498-522.

26. - Maximal 2-components in finite groups, Comm. in Algebra, 4 (1976), 561-594.

27. — Standard components of alternating type I, J. Algebra, 41 (1976), 496514; II, J. Algebra, 47 (1977), 162-179. 
28. R. Solomon, 2-Signalizers in finite groups of alternating type, Comm. in Algebra, (1978), 529-549.

29. T. Yoshida, Character-theoretic transfer, J. Algebra, 52 (1978), 1-38.

Received July 25, 1977 and in revised form October 2, 1978. The first author's work was partly supported by NSF Grant MCS 76-05987 and the second author's work was partly supported by NSF Grant MCS 75-08346.

Stevens Institute of Technology Hoboken, NJ 07030

AND

The Ohio State University Columbus, OH 43210 


\section{PACIFIC JOURNAL OF MATHEMATICS}

EDITORS

DONALD BABBITT (Managing Editor)

University of California

Los Angeles, California 90024

Hugo RossI

University of Utah

Salt Lake City, UT 84112

C. C. MOORE and ANDREW OGG

University of California

Berkeley, CA 94720

\section{J. DUGUNDJI}

Department of Mathematics University of Southern California Los Angeles, California 90007

R. Finn and J. Milgram Stanford University

Stanford, California 94305

\section{ASSOCIATE EDITORS}
E. F. BECKENBACH
B. H. Neumann
F. WOLF
K. YoSHIDA

\section{SUPPORTING INSTITUTIONS}

UNIVERSITY OF BRITISH COLUMBIA

CALIFORNIA INSTITUTE OF TECHNOLOGY

UNIVERSITY OF CALIFORNIA

MONTANA STATE UNIVERSITY

UNIVERSITY OF NEVADA, RENO

NEW MEXICO STATE UNIVERSITY

OREGON STATE UNIVERSITY

UNIVERSITY OF OREGON
UNIVERSITY OF SOUTHERN CALIFORNIA

STANFORD UNIVERSITY

UNIVERSITY OF HAWAII

UNIVERSITY OF TOKYO

UNIVERSITY OF UTAH

WASHINGTON STATE UNIVERSITY

UNIVERSITY OF WASHINGTON 


\section{Pacific Journal of Mathematics}

\section{Vol. 83, No. 1 \\ March, 1979}

Richard Neal Ball, Topological lattice-ordered groups ............... 1

Stephen Berman, On the low-dimensional cohomology of some

infinite-dimensional simple Lie algebras .................. 27

R. P. Boas and Gerald Thomas Cargo, Level sets of derivatives ......... 37

James K. Deveney and John Nelson Mordeson, Splitting and modularly

perfect fields......................................

Robert Hugh Gilman and Ronald Mark Solomon, Finite groups with small

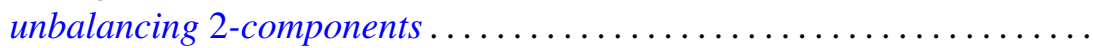

George Grätzer, Andras Hajnal and David C. Kelly, Chain conditions in free products of lattices with infinitary operations..................

Benjamin Rigler Halpern, Periodic points on tori ..................

Dean G. Hoffman and David Anthony Klarner, Sets of integers closed under

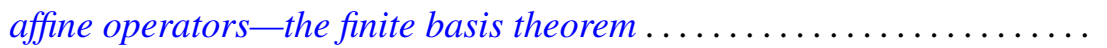

Rudolf-Eberhard Hoffmann, On the sobrification remainder ${ }^{s} X-X \ldots \ldots$

Gerald William Johnson and David Lee Skoug, Scale-invariant

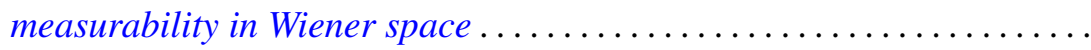

Michael Keisler, Integral representation for elements of the dual of

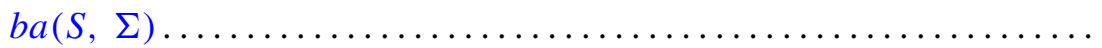

Wayne C. Bell and Michael Keisler, A characterization of the representable Lebesgue decomposition projections ................

Wadi Mahfoud, Comparison theorems for delay differential equations ...

R. Daniel Mauldin, The set of continuous nowhere differentiable functions .

Robert Wilmer Miller and Mark Lawrence Teply, The descending chain condition relative to a torsion theory...

Yoshiomi Nakagami and Colin Eric Sutherland, Takesaki's duality for regular extensions of von Neumann algebras ........ .

William Otis Nowell, Tubular neighborhoods of Hilbert cube manifolds ...

Mohan S. Putcha, Generalization of Lentin's theory of principal solutions of word equations in free semigroups to free product of copies of positive reals under addition

Amitai Regev, A primeness property for central polynomials . ...

Saburou Saitoh, The Rudin kernels on an arbitrary domain. . .

Heinrich Steinlein, Some abstract generalizations of the

Ljusternik-Schnirelmann-Borsuk covering theorem . . . 